\title{
Extended-soft-core baryon-baryon model ESC16. II. Hyperon-nucleon interactions
}

\author{
M. M. Nagels \\ Institute of Mathematics, Astrophysics, and Particle Physics and Radboud University, Nijmegen, The Netherlands \\ Th. A. Rijken \\ Institute of Mathematics, Astrophysics, and Particle Physics University of Nijmegen, Nijmegen, The Netherlands \\ and Nishina Center for Accelerator-Based Science, Institute for Physical and Chemical Research (RIKEN), \\ Wako, Saitama, 351-0198, Japan
}

Y. Yamamoto

Nishina Center for Accelerator-Based Science, Institute for Physical and Chemical Research (RIKEN), Wako, Saitama, 351-0198, Japan

(Received 29 September 2016; revised manuscript received 22 September 2017; published 26 April 2019)

Background: The Nijmegen extended-soft-core (ESC) models describe the nucleon-nucleon (NN), hyperonnucleon $(Y N)$, and the $S=-2$ hyperon-hyperon/nucleon $(Y Y / \Xi N)$ interactions in a unified way using broken SU(3) symmetry. The potentials consist of local and nonlocal potentials due to (i) one-boson exchanges (OBE), which are the members of nonets of pseudoscalar, vector, scalar, and axial-vector mesons, (ii) two pseudoscalar exchange (TPS), (iii) meson-pair exchange (MPE), and (iv) diffractive exchanges. Both the OBE and pair vertices are regulated by Gaussian form factors producing potentials with a soft behavior near the origin. Broken SU(3) symmetry serves to connect the $N N, Y N$, and $Y Y$ channels. In particular, the meson-baryon coupling constants are calculated via SU(3) using the coupling constants of the $N N$ analysis as input. The assignment of the cutoff masses for the baryon-baryon-meson (BBM) vertices is dependent on the SU(3) classification of the exchanged mesons for OBE and a similar scheme for MPE.

Purpose: The $S=-1 Y N$ results are presented from a new version ESC16 of the ESC potential model for baryon-baryon $(B B)$ scattering. The obtained two-body $B B$ potentials are applied to the hyperonic many-body systems as well. Next to the standard ingredients of the ESC models, a contribution of the possible short-range repulsion due to the quark Pauli principle in the $B B$ channels is described in a systematic way for the first time. Methods: Major novel ingredients with respect to the former versions ESC04-ESC08 are the inclusion of (i) short-range Gaussian Odderon potentials corresponding to the odd numbers of gluon exchanges next to the Pomeron potentials due to even gluon exchanges and (ii) short-range repulsion in all $N N, Y N$, and $Y Y$ channels due to Pauli-forbidden six-quark cluster $(0 s)^{6}$ configurations. Further new elements are (i) the extension of the $J^{P C}=1^{++}$axial-vector meson coupling, (ii) the inclusion of the $J^{P C}=1^{+-}$axial-vector mesons, (iii) a completion of the $1 / M$ corrections for the meson-pair-exchange (MPE) potentials, and (iv) the treatment of the scalar $\kappa(861)$ meson within the Gell-Mann-Okubo (GMO) meson-mixing scheme and as a broad meson, like the $\rho(760)$ and $\epsilon(620)$. In contrast to ESC04, we do not consider medium strong flavor-symmetry breaking (FSB) of the coupling constants. The charge-symmetry breaking (CSB) in the $\Lambda p$ and $\Lambda n$ channels, which is an $\mathrm{SU}(2)$ isospin breaking, is included in the OBE, TPS, and MPE potentials. In addition to the usual set of $35 Y N$ data and $3 \Sigma^{+} p$ cross sections from the recent KEK-E289 experiment, we added 11 elastic and inelastic $\Lambda p$ and three elastic $\Sigma^{-} p$ cross sections at higher energy. For the ESC16 model, we performed a simultaneous fit to the combined $N N$ and $Y N$ scattering data, supplied with constraints on the $Y N$ and $Y Y$ interaction originating from the $G$-matrix information on hypernuclei.

Results: The fitting of $N N$ dominates the determination of the couplings and the cutoff masses. Only a few parameters are strongly influenced by the $Y N$ data and by the constraints for the $Y Y$ interactions following from $G$-matrix analyses of hypernuclei and hyperonic matter. Like in the ESC04 model, the obtained octet and singlet coupling constants and $F /(F+D)$ ratios of the model confirm the predictions of the quark-antiquark paircreation (QPC) model with dominance of the ${ }^{3} P_{0}$ mechanism. This not only for the OBE couplings but also for the MPE couplings and $F /(F+D)$ ratios. We obtained within this simultaneous fit $\chi^{2} / N N_{\text {data }}=1.10$ and $\chi^{2} / Y N_{\text {data }}=1.04$. In particular, we were able to fit the precise experimental datum $r_{R}=0.468 \pm 0.010$ for the inelastic $\Sigma^{-} p$ capture ratio at rest very well.

Conclusions: Besides the good results for the fit to the $S=-1$ scattering data, which to a large extent defines the model, also the information of hypernuclear systems, using the $G$-matrix method, is rather important in establishing the complete ESC model. Different versions of the model give somewhat different results for hypernuclei. The reported $G$-matrix calculations are performed for $Y N(\Lambda N$ and $\Sigma N)$ in nuclear matter and also for some hypernuclei. The obtained well depths $\left(U_{\Lambda}, U_{\Sigma}, U_{\Xi}\right)$ reveal distinct features of the ESC model. 
The inclusion of a quark core Pauli repulsion can make the $\Sigma$-nucleus interaction less attractive, as seems to be required by the available experimental evidence. Furthermore, the ESC16 model gives small spin-orbit splittings in $\Lambda$ hypernuclei, which is also indicated by experiment.

\section{DOI: 10.1103/PhysRevC.99.044003}

\section{INTRODUCTION}

This is the second in a series of papers on the extendedsoft-core (ESC) model for low- and intermediate-energy baryon-baryon interactions in the ESC16 version. The nucleon-nucleon interactions are described in Ref. [1]. The first results of ESC08 on the baryon-baryon $(B B)$ channels and applications to hypernuclei were given in the review [2]. Preliminary versions can be found in Refs. [3-5]. With the ESC04 models [6-8], it was shown that a very successful description of the presently available baryon-baryon scattering data could be achieved within the ESC approach to the nuclear force problem. Also, such a description was obtained with meson-baryon coupling parameters, which can be understood rather nicely within the context of the ${ }^{3} P_{0}$ quark-pair creation mechanism $[9,10]$. This mechanism has been shown to be dominant in the framework of lattice QCD [11]. The simultaneous and unified treatment of the $N N$ and $Y N$ channels in ESC04, using broken SU(3), has given already a rather successful potential model for the low- and intermediateenergy baryon-baryon scattering data. Furthermore, the basic ingredients of the model are physically motivated by the quark model (QM) and QCD.

The $G$-matrix calculations showed that basic features of hypernuclear data are also reproduced rather well, improving several weak points of the soft-core one-boson-exchange (OBE) models [12-14]. However, the meson-exchange models seem to be unable to give a positive well depth $U_{\Sigma}$. A second problem was the very small spin-orbit splittings in $\Lambda$ hypernuclei $[15,16]$. In this paper, we extend and refine the ESC model in order to provide improvements and answers to these issues.

First, we list the new ingredients of our version of ESC16, which are more or less in line with the ESC approach as presented so far. In this category, the following additions to the ESC04 model are made for the present ESC16 model:

(i) For the scalar mesons, the mass of the $\varepsilon=f_{0}(620)$ has been lowered, the mixing angle deviates from ideal mixing, and also the $\kappa(861)$ has been treated as a broad meson. This in order to introduce more $\mathrm{SU}(3)$ breaking between $p p$ and $\Sigma^{+} p$.

(ii) For the axial-vector mesons with $J^{P C}=1^{++}$, the $A$ mesons, next to the $\gamma_{5} \gamma_{\mu}$ coupling, and the derivative $\gamma_{5} k_{\mu}$ coupling are exploited.

(iii) The axial-vector mesons with $J^{P C}=1^{+-}$, the $B$ mesons, are included as well. The latter have potentials of the same type as the pseudoscalar mesons but have opposite sign. We notice that now the set of the exchanged quantum numbers for OBE potentials is identical to that for meson-pairexchange (MPE) potentials.

(iv) For the meson exchange, we have included the Brown-Downs-Iddings antisymmetric spin-orbit potentials from pseudoscalar, vector, scalar, and axial meson exchange [17]. (v) We have completed the $1 / M$ corrections for MPE, in particular for the $J^{P C}=1^{++}$and $J^{P C}=1^{+-}$axial pairs. This also leads to new important contributions to the antisymmetric spin-orbit interaction. ${ }^{1}$ (vi) For the diffractive contribution, we have next to the Pomeron exchange ${ }^{2}$ added the Odderon exchange [18]. Whereas in QCD the Pomeron can be associated with colorless even number $(2,4, \ldots)$ of gluon exchanges, the Odderon is associated with the colorless odd number $(3,5, \ldots)$ of gluon exchanges. At low energies, the Pomeron has $J^{P C}=$ $0^{++}$, but the Odderon has $J^{P C}=1^{--}$.

Second, we have opened the possibility to incorporate possible effects of a "structural" or channel-dependent repulsion due to Pauli blocking. This repulsion originates from a forbidden state in the SU(6,FS) quark-cluster model (QCM) $[19,20]$. This is the analog of a well-known effect in $\alpha \alpha$ scattering discovered in the 1960s [21]. This forbidden state is the [51] irrep and this irrep occurs with a large weight in the two $J^{P}=1 / 2^{+}$baryon states in the $\mathrm{SU}(3, \mathrm{~F})$ irreps $\{10\}$ and $\left\{8_{s}\right\}$. These irreps are prominent in the $\Sigma^{+} p\left({ }^{3} S_{1}\right)$ and the $\Sigma N\left({ }^{1} S_{0}\right)$ states, respectively. These are precisely the states where according to, e.g., the $G$-matrix calculations, the ESC models possibly lack some repulsion. This repulsion seems to be indicated by experiment [22,23]. The [51] irrep also occurs in the other $N N, Y N$, and $Y Y$ channels, but with roughly equal weights (see Ref. [19]), apart from a few $S=-2$ channels, e.g., $\Xi N(I=1, S=0)$.

We account for the exceptional repulsion in a phenomenological way, by enhancing the "pure" Pomeron coupling. So, the effective Pomeron repulsion consists of the pure Pomeron-exchange contribution augmented with a fraction of Pauli-blocking repulsion, which varies for the different $B B$ channels. [The other typical quark-cluster effects like onegluon-exchange (OGE) annex quark interchange is in ESC models taken care of by meson exchange.] In this work, we try to determine the strength of this Pauli blocking effect in $B B$ channels. The fit to $N N$ determines the sum of both the pure Pomeron repulsion and the Pauli-blocking repulsion. The fit to $Y N$ determines the fraction of Pauli blocking in it.

The ESC16 model realizes a fusion between the soft-core meson-exchange potentials and QCM aspects of the baryonbaryon interactions and can be called a hybrid ESC model.

\footnotetext{
${ }^{1}$ For the OBE potentials, we have included the Brown-DownsIddings antisymmetric spin-orbit potentials from pseudoscalar, vector, and scalar meson exchange [17]. Also, we derived new antisymmetric spin-orbit contributions from MPE. Since we do not fit $P$ waves for $Y N$, these play no role in the construction of the ESC16 model. Therefore, these potentials will be published elsewhere.

${ }^{2}$ In principle, the off-mass-shell $J=0$ contribution from the tensormeson nonet $A_{2}, K_{2}$, etc., is included with the diffractive soft-core potentials; see, e.g., Refs. [12,13]. Although the couplings are zero in the ESC16 model, we include these potentials in the text for completeness.
} 
The soft-core meson-exchange model has been described in detail in previous papers [6-8]. Therefore, we may refer here to those papers for a description of (a) the physical background, (b) the employed formalism, and (c) the description of the potentials, either in detail or in reference to papers where further information may be obtained. In this paper, we will derive (i) the new OBE potentials employed here for the first time in the context of the ESC model, (ii) the Odderon potentials, and (iii) a derivation of the short-range phenomenology connected to the quark Pauli principle within the context of the SU(3) formalism as used in the Nijmegen potentials. Next to these items, we will also give the new $1 / M$ corrections for the axial-meson-pair-exchange potentials, where we restrict ourselves to the spin-spin and tensor contributions. The $Y N$ symmetric and antisymmetric spin-orbit potentials will be described in another paper.

In Refs. [6,7], a detailed description of the basic features of the ESC models has been given and motivated. Many of these were already present in the Nijmegen soft-core [13] and hard-core [24] OBE models. We refer the reader to these references for the description and discussion of the items such as (broken) SU(3) flavor, charge-symmetry breaking (CSB) in $Y N$, meson mixing in the pseudoscalar, vector, scalar meson SU(3) nonets, and the role of the quark-antiquark pair-creation ${ }^{3} P_{0}$ model for BBM and $B B$ meson-pair couplings. Also, in Ref. [7] one finds a restatement of the goals of our continued investigation of the baryon-baryon systems.

In the soft-core Nijmegen OBE and ESC models, the form factors are taken to be of the Gaussian type. In the (nonrelativistic) QM's, a Gaussian behavior of the form factors for ground-state baryons is most natural. The two-particle branch points, corresponding to e.g., $\pi \pi, \pi \rho, K \rho$, are in the ESC models accounted for by the MPE potentials. Gaussian residue functions are used in regge-pole models for twoparticle reactions at high-energy and low-momentum transfers.

As pointed out in Refs. [6,7], SU(3) symmetry and the QPC model give strong constraints on the coupling parameters. The ${ }^{3} P_{0}$ model also offers the possibility to introduce a scheme for hypercharge breaking à la Gell-Mann-Okubo for the BBM couplings. In order to keep some more flexibility in distinguishing the $N N$ and the $Y N(S=-1)$ channels, such a medium-strong breaking was explored in the NSC97 [14] and ESC04 [7]. In the present study, we do not apply such a breaking. The results show that a scheme of SU(3) symmetric couplings with only mass breaking can give an excellent description of all $B B$ interactions.

The content of this paper is as follows. In Sec. II, we review very briefly the scattering formalism, the LippmannSchwinger equation for the $T$ and $V$ matrices. Similarly, in Sec. III, the $N N$ and $S=-1 Y N$ channels on the isospin and particle basis and the use of the multichannel Schrödinger equation are mentioned. The potentials in momentum and configuration space are defined by referring to the description given in Ref. [6]. Also, SU(3) breaking is reviewed briefly. In Sec. IV, for the OBE potentials, the additions for ESC16 in comparison with the ESC04 model are described. Here, we give the new potentials in momentum and configuration space. Also, the SU(3) structure of the MPE potentials is given and the additions in comparison with the ESC04 model are listed. The latter are the axial $J^{P C}=1^{+-}(\pi \omega)$ pair potentials, which is the content of Appendix C. In Sec. V, the short-range phenomenology is discussed. We derive the incorporation of the exceptional Pauli repulsion, which shows up exceptionally large in the $\mathrm{SU}(3)$ irreps $\{10\}$ and $\left\{8_{s}\right\}$.

In Sec. VI, the simultaneous $N N \oplus Y N \oplus Y Y$ fitting procedure is reviewed. In Sec. VII, the results for the coupling constants and $F /(F+D)$ ratios for OBE and MPE are given. They are discussed and compared with the predictions of the QPC model. Here, also the values of the BBM couplings are displayed for pseudoscalar, vector, scalar, and axial vector mesons.

In Sec. VIII, the $Y N$ results for ESC16 from the combined $N N \oplus Y N \oplus Y Y$ fit are discussed. Here we also discuss the fit to the $Y N$ scattering data. In Sec. IX, the hypernuclear properties of ESC16 are studied through the $G$-matrix calculations for $Y N(\Lambda N, \Sigma N, \Xi N)$ and their partial-wave contributions. Here, the implications of possible three-body effects for the nuclear saturation and baryon well depths are discussed. Also, the $\Lambda \Lambda$ interactions in ESC16 are demonstrated to be consistent with the observed data of ${ }_{\Lambda \Lambda}^{6} \mathrm{He}$. In Sec. X, we finish by a final discussion, draw some conclusions, and present our outlook. In Appendix A, the treatment of the broad mesons is reviewed. In Appendix B, we display the full SU(3) contents of the MPE couplings, and in Appendix C for completeness the $J^{P C}=1^{+-}$axial-pair potentials are given. In Appendix D, the antisymmetric spin-orbit potentials are derived explicitly for strange meson-exchange $K$ and $K^{*}$. Finally, in Appendix E we give the derivation of the antisymmetric spin-orbit potentials.

\section{SCATTERING FORMALISM, THE LIPPMANN-SCHWINGER EQUATION, AND POTENTIALS}

In this paper, we treat the nucleon-nucleon $(N N)$ and hyperon-nucleon $(Y N)$ reactions with strangeness $S=0,-1$. Since in general there are both direct and exchange potentials, the ordering of the baryons in the incoming and outgoing states needs special attention. For keeping this ordering clear, we consider for definiteness the hyperon-nucleon reactions

$$
Y\left(p_{1}, s_{1}\right)+N\left(p_{2}, s_{2}\right) \rightarrow Y^{\prime}\left(p_{1}^{\prime}, s_{1}^{\prime}\right)+N^{\prime}\left(p_{2}^{\prime}, s_{2}^{\prime}\right) .
$$

Like in Ref. [13], whose conventions we will follow in this paper, we will also refer to $Y$ and $Y^{\prime}$ as particles 1 and 3 and to $N$ and $N^{\prime}$ as particles 2 and 4 . The four-momentum of particle $i$ is $p_{i}=\left(E_{i}, \mathbf{p}_{i}\right)$, where $E_{i}=\sqrt{\mathbf{p}_{i}^{2}+M_{i}^{2}}$ and $M_{i}$ is the mass. The transition amplitude matrix $M$ is related to the $S$ matrix via

$$
\langle f|S| i\rangle=\langle f \mid i\rangle-i(2 \pi)^{4} \delta^{4}\left(P_{f}-P_{i}\right)\langle f|M| i\rangle,
$$

where $P_{i}=p_{1}+p_{2}$ and $P_{f}=p_{1}^{\prime}+p_{2}^{\prime}$ represent the total four-momentum for the initial state $|i\rangle$ and the final state $|f\rangle$. The latter refer to the two-particle states, which we normalize in the following way:

$$
\begin{aligned}
\left\langle\mathbf{p}_{1}^{\prime}, \mathbf{p}_{2}^{\prime} \mid \mathbf{p}_{1}, \mathbf{p}_{2}\right\rangle= & (2 \pi)^{3} 2 E\left(\mathbf{p}_{1}\right) \delta^{3}\left(\mathbf{p}_{1}^{\prime}-\mathbf{p}_{1}\right) \\
& \times(2 \pi)^{3} 2 E\left(\mathbf{p}_{2}\right) \delta^{3}\left(\mathbf{p}_{2}^{\prime}-\mathbf{p}_{2}\right) .
\end{aligned}
$$


We follow Sec. II of Ref. [13] in detail. The transformation to the nonrelativistic normalization of the two-particle states leads to states with

$$
\begin{aligned}
& \left(\mathbf{p}_{1}^{\prime}, s_{1}^{\prime} ; \mathbf{p}_{2}^{\prime}, s_{2}^{\prime} \mid \mathbf{p}_{1}, s_{1} ; \mathbf{p}_{2}, s_{2}\right) \\
& \quad=(2 \pi)^{6} \delta^{3}\left(\mathbf{p}_{1}^{\prime}-\mathbf{p}_{1}\right) \delta^{3}\left(\mathbf{p}_{2}^{\prime}-\mathbf{p}_{2}\right) \delta_{s_{1}^{\prime}, s_{1}} \delta_{s_{2}^{\prime}, s_{1}} .
\end{aligned}
$$

For these states, we define the $T$ matrix by

$$
(f|T| i)=\left\{4 M_{34}\left(E_{3}+E_{4}\right)\right\}^{-\frac{1}{2}}\langle f|M| i\rangle\left\{4 M_{12}\left(E_{1}+E_{2}\right)\right\}^{-\frac{1}{2}},
$$

which satisfies the Lippmann-Schwinger equation [13]

$$
\begin{aligned}
(3,4|T| 1,2)= & (3,4|V| 1,2)+\frac{1}{(2 \pi)^{3}} \sum_{n} \int d^{3} k_{n} \\
& \times\left(3,4|V| n_{1}, n_{2}\right) \frac{2 M_{n_{1}, n_{2}}}{\mathbf{p}_{n}^{2}-\mathbf{k}_{n}^{2}+i \varepsilon}\left(n_{1}, n_{2}|T| 1,2\right),
\end{aligned}
$$

and where analogously to Eq. (2.5) the potential $V$ is defined as

$$
(f|V| i)=\left\{4 M_{34}\left(E_{3}+E_{4}\right)\right\}^{-\frac{1}{2}}\langle f|W| i\rangle\left\{4 M_{12}\left(E_{1}+E_{2}\right)\right\}^{-\frac{1}{2}} .
$$

Above, we denoted the initial- and final-state c.m. momenta by $\mathbf{p}_{i}$ and $\mathbf{p}_{f}$. Using rotational invariance and parity conservation, we expand the $T$ matrix, which is a $4 \times 4$ matrix in Paulispinor space, into a complete set of Pauli-spinor invariants $[13,25]$ :

$$
T=\sum_{i=1}^{8} T_{i}\left(\mathbf{p}_{f}^{2}, \mathbf{p}_{i}^{2}, \mathbf{p}_{i} \cdot \mathbf{p}_{f}\right) P_{i}
$$

Introducing

$$
\mathbf{q}=\frac{1}{2}\left(\mathbf{p}_{f}+\mathbf{p}_{i}\right), \quad \mathbf{k}=\mathbf{p}_{f}-\mathbf{p}_{i}, \quad \mathbf{n}=\mathbf{p}_{i} \times \mathbf{p}_{f}=\mathbf{q} \times \mathbf{k}
$$

with, of course, $\mathbf{n}=\mathbf{q} \times \mathbf{k}$, we choose for the operators $P_{i}$ in spin space

$$
\begin{aligned}
& P_{1}=1, \\
& P_{2}=\sigma_{1} \cdot \sigma_{2}, \\
& P_{3}=\left(\sigma_{1} \cdot \mathbf{k}\right)\left(\sigma_{2} \cdot \mathbf{k}\right)-\frac{1}{3}\left(\sigma_{1} \cdot \sigma_{2}\right) \mathbf{k}^{2}, \\
& P_{4}=\frac{i}{2}\left(\sigma_{1}+\sigma_{2}\right) \cdot \mathbf{n}, \\
& P_{5}=\left(\sigma_{1} \cdot \mathbf{n}\right)\left(\sigma_{2} \cdot \mathbf{n}\right), \\
& P_{6}=\frac{i}{2}\left(\sigma_{1}-\sigma_{2}\right) \cdot \mathbf{n}, \\
& P_{7}=\left(\sigma_{1} \cdot \mathbf{q}\right)\left(\sigma_{2} \cdot \mathbf{k}\right)+\left(\sigma_{1} \cdot \mathbf{k}\right)\left(\sigma_{2} \cdot \mathbf{q}\right), \\
& P_{8}=\left(\sigma_{1} \cdot \mathbf{q}\right)\left(\sigma_{2} \cdot \mathbf{k}\right)-\left(\sigma_{1} \cdot \mathbf{k}\right)\left(\sigma_{2} \cdot \mathbf{q}\right) .
\end{aligned}
$$

Here, we follow Refs. [13,25], except that we have chosen here $P_{3}$ to be a purely tensor-force operator.

Similarly to (2.9), the potentials are expanded as

$$
V=\sum_{i=1}^{6} V_{i}\left(\mathbf{k}^{2}, \mathbf{q}^{2}\right) P_{i}
$$

The potentials in configuration space are described in Paulispinor space as follows:

$$
\begin{aligned}
V(r)= & V_{C}(r)+V_{\sigma}(r) \boldsymbol{\sigma}_{1} \cdot \boldsymbol{\sigma}_{2}+V_{T}(r) S_{12}+V_{\mathrm{SLS}}(r) \mathbf{L} \cdot \mathbf{S}_{+} \\
& +V_{\mathrm{ALS}}(r) \mathbf{L} \cdot \mathbf{S}_{-}+V_{Q}(r) Q_{12},
\end{aligned}
$$

where $\mathbf{S}_{ \pm}=\left(\sigma_{1} \pm \boldsymbol{\sigma}_{2}\right) / 2$; see, e.g., Ref. [13] for a definition of the operators $S_{12}$ and $Q_{12}$.

\section{CHANNELS, POTENTIALS, AND SU(3) SYMMETRY}

\section{A. Channels and potentials}

On the physical particle basis, there are three charge $N N$ channels:

$$
q=+2,+1,0: p p \rightarrow p p, p n \rightarrow p n, n n \rightarrow n n .
$$

Similarly, there are four charge $Y N$ channels:

$$
\begin{aligned}
& q=+2: \Sigma^{+} p \rightarrow \Sigma^{+} p, \\
& q=+1:\left(\Lambda p, \Sigma^{+} n, \Sigma^{0} p\right) \rightarrow\left(\Lambda p, \Sigma^{+} n, \Sigma^{0} p\right), \\
& q=0:\left(\Lambda n, \Sigma^{0} n, \Sigma^{-} p\right) \rightarrow\left(\Lambda n, \Sigma^{0} n, \Sigma^{-} p\right), \\
& q=-1: \Sigma^{-} n \rightarrow \Sigma^{-} n .
\end{aligned}
$$

Like in Refs. [13,14], the potentials are calculated on the isospin basis. For $S=0$ nucleon-nucleon systems, there are two isospin channels, namely $I=1$ and $I=0$. For $S=-1$ hyperon-nucleon systems, there are also two isospin channels: (i) $I=\frac{1}{2}:(\Lambda N, \Sigma N \rightarrow \Lambda N, \Sigma N)$ and (ii) $I=\frac{3}{2}: \Sigma N \rightarrow$ $\Sigma N$.

For the OBE part of the potentials, the treatment of SU(3) for the BBM interaction Lagrangians and the coupling coefficients of the OBE graphs have been given in detail in previous work of the Nijmegen group, e.g., Refs. [13,14]. For the TME and the MPE parts, the calculation of the coupling coefficients has been exposed in our paper on the ESC04 model [7]. There we described the method of an automatic computerized calculation of these coefficients by exploiting the Cartesian-octet representation.

Also, in this work, we do not solve the LippmannSchwinger equation but the multichannel Schrödinger equation in configuration space, completely analogous to Ref. [13]. The multichannel Schrödinger equation for the configuration space potential is derived from the Lippmann-Schwinger equation through the standard Fourier transform, and the equation for the radial wave function is found to be of the form [13]

$$
u_{l, j}^{\prime \prime}+\left(p_{i}^{2} \delta_{i, j}-A_{i, j}\right) u_{l, j}-B_{i, j} u_{l, j}^{\prime}=0,
$$

where $A_{i, j}$ contains the potential, nonlocal contributions, and the centrifugal barrier, while $B_{i, j}$ is only present when nonlocal contributions are included. The solution in the presence of open and closed channels is given, for example, in Ref. [26]. The inclusion of the Coulomb interaction in the configurationspace equation is well known and included in the evaluation of the scattering matrix.

The momentum space and configuration space potentials for the ESC models have been described in Ref. [6] for baryon-baryon in general. Here, we will only give the new contributions to these potentials, both in momentum and configuration space. 


\section{B. SU(3) symmetry and symmetry breaking, plus form factors}

The treatment of the mass differences among the baryons is handled in the same way as for ESC04, which is exactly that of other Nijmegen models [13,14,24]. Also, exchange potentials related to strange meson exchange $K, K^{*}$ can be found in these references.

The breaking of SU(3) symmetry occurs in several places. The physical masses of the baryons and mesons are used. Noticable is the $\mathrm{SU}(2) \subset \mathrm{SU}(3)$ breaking due to $\Lambda-\Sigma^{0}$ mixing [27]. This $\Lambda-\Sigma^{0}$ mixing leads also to a nonzero coupling of the $\Lambda$ to the other $I=1$ mesons: $\rho$ (760), $a_{0}(980), a_{1}(1270)$, as well as to the $I=1$ pairs. For the details of these OBE couplings, see, e.g., Ref. [14], Eqs. (2.15)-(2.17). Like in ESC04, the corresponding so-called CSB potentials are included in the ESC16 model for OBE, TME, and MPE.

The medium-strong SU(3) symmetry breaking of the BBM coupling constants is not tried in ESC16. In the ESC04 model, this was considered optional and regulated by the ${ }^{3} P_{0}$ model by a differentiation between the $s \bar{s}$-quark pair and the creation of a nonstrange quark-antiquark pair. Of course, we could contemplate such an option here but we did not investigate this option.

The baryon mass differences in the intermediate states for TME and MPE potentials have been neglected for $Y N$ scattering. This, although possible in principle, becomes rather laborious and is not expected to change the characteristics of the baryon-baryon potentials much.

Also in this work, like in ESC04 [6-8] and NSC97 models [14], the form factors depend on the SU(3) assignment of the mesons. In principle, we introduce form factor masses, i.e., cutoffs, $\Lambda_{8}$ and $\Lambda_{1}$ for the $\{8\}$ and $\{1\}$ members of each meson nonet, respectively. In the application to $Y N$ and $Y Y$, we could allow for SU(3) breaking by using different cutoffs for the strange mesons $K, K^{*}$, and $\kappa$. However, in the ESC16 model, we do not exploit this possible breaking but assign for the strange $I=1 / 2$ mesons the same cutoff as for the $I=1$ mesons. Moreover, for the $I=0$ mesons, we assign the cutoffs as if there were no meson mixing. For example, we assign $\Lambda_{1}$ for the dominant singlet mesons $\eta^{\prime}, \omega, \epsilon$, and $\Lambda_{8}$ for $\eta, \phi, S^{*}$, etc. This means a very slight form of SU(3) symmetry breaking.

\section{NEW POTENTIALS IN ESC16}

The OBE potentials in ESC16 are those contained already in ESC04 [6,7], and some new additional contributions. The additions to the OBE potentials with regard to the ESC04 models consist of the following elements: (i) extension of the baryon-baryon-meson vertex of the axial-vector mesons $\left(J^{P C}=1^{++}\right)$by adding the derivative coupling and (ii) inclusion of the axial-vector mesons of the second kind, having $J^{P C}=1^{+-}$. In Ref. [1], the potentials for nonstrange meson exchange have been given. Here, we list the additions and the basic potentials for meson exchange with nonzero strangeness.

\section{A. Additions to the OBE potentials in ESC16}

The interaction Hamiltonian densities for the new couplings are as follows: (a) Axial-vector-meson exchange $\left(J^{P C}=1^{++}\right.$, first kind):

$$
\mathcal{H}_{A}=g_{A}\left[\bar{\psi} \gamma_{\mu} \gamma_{5} \psi\right] \phi_{A}^{\mu}+\frac{i f_{A}}{\mathcal{M}}\left[\bar{\psi} \gamma_{5} \psi\right] \partial_{\mu} \phi_{A}^{\mu} .
$$

In ESC04, the $g_{A}$ coupling was included, but not the derivative $f_{A}$ coupling.

(b) Axial-vector-meson exchange $\left(J^{P C}=1^{+-}\right.$, second kind):

$$
\mathcal{H}_{B}=\frac{i f_{B}}{m_{B}}\left[\bar{\psi} \sigma_{\mu \nu} \gamma_{5} \psi\right] \partial_{\nu} \phi_{B}^{\mu},
$$

where $m_{B}$ is the mass $b_{1}(1235)$. In ESC04, this coupling was not included. Like for the axial-vector mesons of the first kind, we include a SU(3) nonet with members $b_{1}(1235), h_{1}(1170), h_{1}^{\prime}(1380)$. In the quark model, they are $Q \bar{Q}\left({ }^{1} P_{1}\right)$ states.

The inclusion of the gaussian form factors is discussed in previous papers [13] and reviewed in Ref. [1]. For the approximations made in deriving the potentials from the relativistic Born approximation, we refer also to Ref. [1]. Because of these approximations, the dependence on $\mathbf{q}^{2}$ is linearized, and we write

$$
V_{i}\left(\mathbf{k}^{2}, \mathbf{q}^{2}\right)=V_{i a}\left(\mathbf{k}^{2}\right)+V_{i b}\left(\mathbf{k}^{2}\right)\left(\mathbf{q}^{2}+\frac{1}{4} \mathbf{k}^{2}\right),
$$

where $i=1-8$. The combination $\left(\mathbf{q}^{2}+\mathbf{k}^{2} / 4\right)$ leads to a purely nonlocal potential. The additional OBE potentials are obtained in the standard way; see Refs. $[12,13]$. We write the potential functions $V_{i}$ of (2.11) in the form

$$
V_{i}\left(\mathbf{k}^{2}, \mathbf{q}^{2}\right)=\sum_{X} \Omega_{i}^{(X)}\left(\mathbf{k}^{2}\right) \cdot \Delta^{(X)}\left(\mathbf{k}^{2}, m^{2}, \Lambda^{2}\right),
$$

where $m$ denotes the mass of the meson, $\Lambda$ is the cutoff in the Gaussian form factor, and $X=P, V, S, A, B$, and $D(P$, pseudoscalar; $V$, vector; $S$, scalar; $A$, axial vector; $B$, axial vector; and $D$, Pomeron-Odderon). For meson exchange, the propagator $\Delta^{(X)}$ function is

$$
\Delta^{(X)}\left(\mathbf{k}^{2}, m^{2}, \Lambda^{2}\right)=e^{-\mathbf{k}^{2} / \Lambda^{2}} /\left(\mathbf{k}^{2}+m^{2}\right) .
$$

For $X=S, A$, we have included in the propagator a zero by the factor $\left(1-\mathbf{k}^{2} / U^{2}\right)$, with $U=750 \mathrm{MeV}[6,7]$. In the case $X=D$, the propagator is replaced by

$$
\Delta^{(D)}\left(\mathbf{k}^{2}, m^{2}, \Lambda^{2}\right)=\frac{1}{\mathcal{M}^{2}} e^{-\mathbf{k}^{2} / 4 m_{D}^{2}} .
$$

Here, $\mathcal{M}$ is a universal scaling mass, taken to be the proton mass, which we also use in the derivative couplings above, as well as in the $f_{V}$ and $f_{A}$ couplings of the vector mesons.

\section{B. Meson exchange with nonzero strangeness $(\Delta Y \neq 0)$}

For the nonstrange mesons, the mass differences at the vertices are neglected, and we take at the $Y Y M$ and the $N N M$ vertex the average hyperon and the average nucleon mass respectively. This implies that we do not include contributions to the Pauli invariants $P_{7}$ and $P_{8}$. These exchanges lead to the so-called exchange potentials. For the invariants $O_{1}, \ldots, O_{6}$, the expressions analogous to those for the nonstrange mesons given above apply. This with the amendments that (i) in 
momentum and configuration space there is a complete symmetric appearance of $M_{Y}$ and $M_{N}$, (ii) in configuration space there appears the baryon-exchange operator $\mathcal{P}=-\mathcal{P}_{x} \mathcal{P}_{\sigma}$ operator, and (iii) for the antisymmetric spin-orbit potential $\mathcal{P} \rightarrow \mathcal{P}_{x}$. The details are given in Appendix D. Therefore, the $\Omega_{i}^{(X)}$ for these potentials can be obtained from those given in Ref. [1], Eqs. (4.14)-(4.18), by replacing both $M_{Y}$ and $M_{N}$ by $\left(M_{Y} M_{N}\right)^{1 / 2}$. Furthermore, in the case of the vector and axial-B mesons, the Proca formalism [28] is used, which gives for the vector mesons non-negligible contributions from the second part of the vector-meson propagator $\left(k_{\mu} k_{v} / \mathrm{m}^{2}\right)$ of the $K^{*}$ meson,

$$
-V_{i}^{K^{*}}=V_{i}^{(V)}-\frac{\left(M_{3}-M_{1}\right)\left(M_{4}-M_{2}\right)}{m^{2}} V_{i}^{(S)},
$$

where in $V_{i}^{(S)}$ the vector-meson couplings have to be used, and $M_{Y}$ and $M_{N}$ must be replaced by $\left(M_{Y} M_{N}\right)^{1 / 2}$. In Eq. (4.7), $M_{1}=M_{4}=M_{Y}$ and $M_{2}=M_{3}=M_{N}$. For the axial $A$ mesons, we use the $B$-field formalism (see Ref. [1] Appendix A), and there is no second term in the propagator.

For the mesons with nonzero strangeness, $K, K^{*}, \kappa, K_{A}$, and $K_{B}$, the mass differences at the vertices are not neglected, and we take into account at the YNM vertices the differences between the average hyperon and the average nucleon mass. This implies that we do include contributions to the Pauli invariants $P_{8}$. There do not occur contributions to $P_{7}$. Furthermore, mass differences in the $Y N$ propagation are included via a meson mass corrections in the strange-meson propagators:

(a) Pseudoscalar $K$-meson exchange:

$$
\begin{aligned}
& \Omega_{2 a}^{(P)}=-f_{13}^{P} f_{24}^{P}\left(\frac{\mathbf{k}^{2}}{3 m_{\pi^{+}}^{2}}\right), \quad \Omega_{3 a}^{(P)}=-f_{13}^{P} f_{24}^{P}\left(\frac{1}{m_{\pi^{+}}^{2}}\right), \\
& \Omega_{2 b}^{(P)}=+f_{13}^{P} f_{24}^{P}\left(\frac{\mathbf{k}^{2}}{6 m_{\pi^{+}}^{2}}\right), \quad \Omega_{3 b}^{(P)}=+f_{13}^{P} f_{24}^{P}\left(\frac{1}{2 m_{\pi^{+}}^{2} M_{Y} M_{N}}\right) .
\end{aligned}
$$

(b) Vector-meson $K^{*}$-exchange:

$$
\begin{aligned}
& \Omega_{1 a}^{(V)}=\left\{g_{13}^{V} g_{24}^{V}\left(1-\frac{\mathbf{k}^{2}}{2 M_{Y} M_{N}}\right)-\left(g_{13}^{V} f_{24}^{V}+f_{13}^{V} g_{24}^{V}\right) \frac{\mathbf{k}^{2}}{4 \mathcal{M} \sqrt{M_{Y} M_{N}}}+f_{13}^{V} f_{24}^{V} \frac{\mathbf{k}^{4}}{16 \mathcal{M}^{2} M_{Y} M_{N}}\right\}, \\
& \Omega_{1 b}^{(V)}=g_{13}^{V} g_{24}^{V}\left(\frac{3}{2 M_{Y} M_{N}}\right), \quad \Omega_{2 a}^{(V)}=-\frac{2}{3} \mathbf{k}^{2} \Omega_{3 a}^{(V)}, \quad \Omega_{2 b}^{(V)}=-\frac{2}{3} \mathbf{k}^{2} \Omega_{3 b}^{(V)}, \\
& \Omega_{3 a}^{(V)}=\left\{\left(g_{13}^{V} g_{24}^{V}+\left(g_{13}^{V} f_{24}^{V}+g_{24}^{V} f_{13}^{V}\right) \frac{\sqrt{M_{Y} M_{N}}}{\mathcal{M}}\right)-f_{13}^{V} f_{24}^{V} \frac{\mathbf{k}^{2}}{8 \mathcal{M}^{2}}\right\} /\left(4 M_{Y} M_{N}\right), \\
& \Omega_{3 b}^{(V)}=-\left(g_{13}^{V}+f_{13}^{V} \frac{M_{Y}}{\mathcal{M}}\right)\left(g_{24}^{V}+f_{24}^{V} \frac{M_{N}}{\mathcal{M}}\right) /\left(8 M_{Y}^{2} M_{N}^{2}\right), \\
& \Omega_{4}^{(V)}=-\left\{12 g_{13}^{V} g_{24}^{V}+8\left(g_{13}^{V} f_{24}^{V}+f_{13}^{V} g_{24}^{V}\right) \frac{\sqrt{M_{Y} M_{N}}}{\mathcal{M}_{13}}-f_{13}^{V} f_{24}^{V} \frac{3 \mathbf{k}^{2}}{\mathcal{M}^{2}}\right\} /\left(8 M_{Y} M_{N}\right), \\
& \Omega_{5}^{(V)}=-\left\{g_{13}^{V} g_{24}^{V}+4\left(g_{13}^{V} f_{24}^{V}+f_{13}^{V} g_{24}^{V}\right) \frac{\sqrt{M_{Y} M_{N}}}{\mathcal{M}}+8 f_{13}^{V} f_{24}^{V} \frac{M_{Y} M_{N}}{\mathcal{M}^{2}}\right\} /\left(16 M_{Y}^{2} M_{N}^{2}\right), \\
& \Omega_{6}^{(V)}=-\left\{\left(g_{13}^{V} f_{24}^{V}-f_{13}^{V} g_{24}^{V}\right) \frac{1}{\sqrt{\mathcal{M}^{2} M_{Y} M_{N}}}\right\}
\end{aligned}
$$

(c) Scalar-meson $\kappa$ exchange:

$$
\begin{aligned}
& \Omega_{1 a}^{(S)}=-g_{13}^{S} g_{24}^{S}\left(1+\frac{\mathbf{k}^{2}}{4 M_{Y} M_{N}}\right), \quad \Omega_{1 b}^{(S)}=+g_{13}^{S} g_{24}^{S} \frac{1}{2 M_{Y} M_{N}}, \\
& \Omega_{4}^{(S)}=-g_{13}^{S} g_{24}^{S} \frac{1}{2 M_{Y} M_{N}}, \quad \Omega_{5}^{(S)}=g_{13}^{S} g_{24}^{S} \frac{1}{16 M_{Y}^{2} M_{N}^{2}}, \quad \Omega_{6}^{(S)}=0 .
\end{aligned}
$$

(d) Axial-vector $K_{1 A}$ exchange $J^{P C}=1^{++}$:

$$
\begin{aligned}
& \Omega_{2}^{(A)}=-g_{13}^{A} g_{24}^{A}\left[1-\frac{2 \mathbf{k}^{2}}{3 M_{Y} M_{N}}\right]+\left[\left(g_{13}^{A} f_{24}^{A}+f_{13}^{A} g_{24}^{A}\right) \frac{\sqrt{M_{Y} M_{N}}}{\mathcal{M}}-f_{13}^{A} f_{24}^{A} \frac{\mathbf{k}^{2}}{2 \mathcal{M}^{2}}\right] \frac{\mathbf{k}^{2}}{6 M_{Y} M_{N}}, \\
& \Omega_{2 b}^{(A)}=-g_{13}^{A} g_{24}^{A}\left(\frac{3}{2 M_{Y} M_{N}}\right),
\end{aligned}
$$




$$
\begin{aligned}
& \Omega_{3}^{(A)}=-g_{13}^{A} g_{24}^{A}\left[\frac{1}{4 M_{Y} M_{N}}\right]+\left[\left(g_{13}^{A} f_{24}^{A}+f_{13}^{A} g_{24}^{A}\right) \frac{\sqrt{M_{Y} M_{N}}}{\mathcal{M}}-f_{13}^{A} f_{24}^{A} \frac{\mathbf{k}^{2}}{2 \mathcal{M}^{2}}\right] \frac{1}{2 M_{Y} M_{N}}, \\
& \Omega_{4}^{(A)}=-g_{13}^{A} g_{24}^{A}\left[\frac{1}{2 M_{Y} M_{N}}\right], \quad \Omega_{5}^{(A)^{\prime}}=-g_{13}^{A} g_{24}^{A}\left[\frac{2}{M_{Y} M_{N}}\right], \quad \Omega_{6}^{(A)}=0 .
\end{aligned}
$$

Here, we used the $B$-field description with $\alpha_{r}=1$; see Ref. [1], Appendix A. The detailed treatment of the potential proportional to $P_{5}^{\prime}$, i.e., with $\Omega_{5}^{(A)^{\prime}}$, is given in Ref. [1], Appendix B.

(e) Axial-vector $K_{1 B^{-}}$exchange $J^{P C}=1^{+-}$:

$$
\begin{array}{ll}
\Omega_{2 a}^{(B)}=+f_{13}^{B} f_{24}^{B} \frac{4 M_{N} M_{Y}}{m_{B}^{2}}\left(1-\frac{\mathbf{k}^{2}}{4 M_{Y} M_{N}}\right)\left(\frac{\mathbf{k}^{2}}{12 M_{Y} M_{N}}\right), & \Omega_{2 b}^{(B)}=+f_{13}^{B} f_{24}^{B} \frac{4 M_{N} M_{Y}}{m_{B}^{2}}\left(\frac{\mathbf{k}^{2}}{8 M_{Y}^{2} M_{N}^{2}}\right), \\
\Omega_{3 a}^{(B)}=+f_{13}^{B} f_{24}^{B} \frac{4 M_{N} M_{Y}}{m_{B}^{2}}\left(1-\frac{\mathbf{k}^{2}}{4 M_{Y} M_{N}}\right),\left(\frac{1}{4 M_{Y} M_{N}}\right), & \Omega_{3 b}^{(B)}=+f_{13}^{B} f_{24}^{B} \frac{4 M_{N} M_{Y}}{m_{B}^{2}}\left(\frac{3}{8 M_{Y}^{2} M_{N}^{2}}\right),
\end{array}
$$

As in Ref. [13], in the derivation of the expressions for $\Omega_{i}^{(X)}$, given above, $M_{Y}$ and $M_{N}$ denote the mean hyperon and nucleon mass, respectively, $M_{Y}=\left(M_{1}+M_{3}\right) / 2$ and $M_{N}=\left(M_{2}+M_{4}\right) / 2$, and $m$ denotes the mass of the exchanged meson. Moreover, the approximation $1 / M_{N}^{2}+1 / M_{Y}^{2} \approx 2 /\left(M_{N} M_{Y}\right)$ is used, which is rather good since the mass differences between the baryons are not large.

\section{One-boson-exchange interactions in configuration space I}

In configuration space, the $B B$ interactions are described by potentials of the general form

$$
\begin{aligned}
V= & \left\{V_{C}(r)+V_{\sigma}(r) \boldsymbol{\sigma}_{1} \cdot \boldsymbol{\sigma}_{2}+V_{T}(r) S_{12}+V_{S O}(r) \mathbf{L} \cdot \mathbf{S}+V_{Q}(r) Q_{12}\right. \\
& \left.+V_{\mathrm{ASO}}(r) \frac{1}{2}\left(\sigma_{1}-\boldsymbol{\sigma}_{2}\right) \cdot \mathbf{L}-\frac{1}{2 M_{Y} M_{N}}\left(\nabla^{2} V^{n . l .}(r)+V^{n . l .}(r) \nabla^{2}\right)\right\} \mathcal{P}, \\
V^{n . l .}= & \left\{\varphi_{C}(r)+\varphi_{\sigma}(r) \boldsymbol{\sigma}_{1} \cdot \boldsymbol{\sigma}_{2}+\varphi_{T}(r) S_{12}\right\},
\end{aligned}
$$

where

$$
\begin{aligned}
S_{12} & =3\left(\sigma_{1} \cdot \hat{r}\right)\left(\sigma_{2} \cdot \hat{r}\right)-\left(\sigma_{1} \cdot \sigma_{2}\right), \\
Q_{12} & =\frac{1}{2}\left[\left(\sigma_{1} \cdot \mathbf{L}\right)\left(\sigma_{2} \cdot \mathbf{L}\right)+\left(\sigma_{2} \cdot \mathbf{L}\right)\left(\sigma_{1} \cdot \mathbf{L}\right)\right] .
\end{aligned}
$$

For the basic functions for the Fourier transforms with Gaussian form factors, we refer to Refs. [12,13]. For the details of the Fourier transform for the potentials with $P_{5}^{\prime}$, which occur in the case of the axial-vector mesons with $J^{P C}=1^{++}$, we refer to Ref. [1], Appendix B.

(a) Pseudoscalar-meson $K$ exchange:

$$
\begin{aligned}
V_{P S}(r) & =\frac{m}{4 \pi}\left[f_{13}^{P} f_{24}^{P}\left(\frac{m^{2}}{m_{\pi^{+}}^{2}}\right)\left(\frac{1}{3}\left(\sigma_{1} \cdot \sigma_{2}\right) \phi_{C}^{1}+S_{12} \phi_{T}^{0}\right)\right] \mathcal{P}, \\
V_{P S}^{n . l .}(r) & =-\frac{m}{4 \pi}\left[f_{13}^{P} f_{24}^{P}\left(\frac{m^{2}}{2 m_{\pi^{+}}^{2}}\right)\left(\frac{1}{3}\left(\sigma_{1} \cdot \sigma_{2}\right) \phi_{C}^{1}+S_{12} \phi_{T}^{0}\right)\right] \mathcal{P} .
\end{aligned}
$$

(b) Vector-meson $K^{*}$ exchange:

$$
\begin{aligned}
V_{V}(r)= & \frac{m}{4 \pi}\left[\left\{g_{13}^{V} g_{24}^{V}\left[\phi_{C}^{0}+\frac{m^{2}}{2 M_{Y} M_{N}} \phi_{C}^{1}\right]+\left(g_{13}^{V} f_{24}^{V}+f_{13}^{V} g_{24}^{V}\right) \frac{m^{2}}{4 \mathcal{M} \sqrt{M_{Y} M_{N}}} \phi_{C}^{1}+f_{13}^{V} f_{24}^{V} \frac{m^{4}}{16 \mathcal{M}^{2} M_{Y} M_{N}} \phi_{C}^{2}\right\}\right. \\
& +\frac{m^{2}}{6 M_{Y} M_{N}}\left\{\left[g_{13}^{V} g_{24}^{V}+\left(g_{13}^{V} f_{13}^{V}+g_{24}^{V} f_{13}^{V}\right) \frac{\sqrt{M_{Y} M_{N}}}{\mathcal{M}}+f_{13}^{V} f_{24}^{V} \frac{M_{Y} M_{N}}{\mathcal{M}^{2}}\right] \phi_{C}^{1}+f_{13}^{V} f_{24}^{V} \frac{m^{2}}{8 \mathcal{M}^{2}} \phi_{C}^{2}\right\}\left(\sigma_{1} \cdot \sigma_{2}\right) \\
& -\frac{m^{2}}{4 M_{Y} M_{N}}\left\{\left[g_{13}^{V} g_{24}^{V}+\left(g_{13}^{V} f_{24}^{V}+g_{24}^{V} f_{13}^{V}\right) \frac{\sqrt{M_{Y} M_{N}}}{\mathcal{M}}\right) \phi_{T}^{0}+f_{13}^{V} f_{24}^{V} \frac{m^{2}}{8 \mathcal{M}^{2}} \phi_{T}^{1}\right\} S_{12}
\end{aligned}
$$




$$
\begin{aligned}
& -\frac{m^{2}}{M_{Y} M_{N}}\left\{\left[\frac{3}{2} g_{13}^{V} g_{24}^{V}+\left(g_{13}^{V} f_{24}^{V}+f_{13}^{V} g_{24}^{V}\right) \frac{\sqrt{M_{Y} M_{N}}}{\mathcal{M}}\right] \phi_{S O}^{0}+\frac{3}{8} f_{13}^{V} f_{24}^{V} \frac{m^{2}}{\mathcal{M}^{2}} \phi_{S O}^{1}\right\} \mathbf{L} \cdot \mathbf{S} \\
& +\frac{m^{4}}{16 M_{Y}^{2} M_{N}^{2}}\left\{\left[g_{13}^{V} g_{24}^{V}+4\left(g_{13}^{V} f_{24}^{V}+f_{13}^{V} g_{24}^{V}\right) \frac{\sqrt{M_{Y} M_{N}}}{\mathcal{M}}+8 f_{13}^{V} f_{24}^{V} \frac{M_{Y} M_{N}}{\mathcal{M}^{2}}\right]\right\} \\
& \left.\times \frac{3}{(m r)^{2}} \phi_{T}^{0} Q_{12}+\frac{m^{2}}{M_{Y} M_{N}}\left\{\left(g_{13}^{V} f_{24}^{V}-f_{13}^{V} g_{24}^{V}\right) \frac{\sqrt{M_{Y} M_{N}}}{\mathcal{M}} \phi_{S O}^{0}\right\} \frac{1}{2}\left(\sigma_{1}-\sigma_{2}\right) \cdot \mathbf{L} \mathcal{P}_{\sigma}\right] \mathcal{P}, \\
V_{V}^{n . l .}(r)= & \frac{m}{4 \pi}\left[\frac{3}{2} g_{13}^{V} g_{24}^{V} \phi_{C}^{0}+\frac{m^{2}}{6 M_{Y} M_{N}}\left\{\left[\left(g_{13}^{V}+f_{13}^{V} \frac{\sqrt{M_{Y} M_{N}}}{\mathcal{M}}\right)\left(g_{24}^{V}+f_{24}^{V} \frac{\sqrt{M_{Y} M_{N}}}{\mathcal{M}}\right)\right] \phi_{C}^{1}\right\}\left(\sigma_{1} \cdot \boldsymbol{\sigma}_{2}\right)\right. \\
& \left.-\frac{m^{2}}{4 M_{Y} M_{N}}\left\{\left[\left(g_{13}^{V}+f_{13}^{V} \frac{\sqrt{M_{Y} M_{N}}}{\mathcal{M}}\right)\left(g_{24}^{V}+f_{24}^{V} \frac{\sqrt{M_{Y} M_{N}}}{\mathcal{M}}\right)\right] \phi_{T}^{0}\right\} S_{12}\right] .
\end{aligned}
$$

(c) Scalar-meson $\kappa$-exchange:

$$
\begin{aligned}
V_{S}(r) & =-\frac{m}{4 \pi}\left[g_{13}^{S} g_{24}^{S}\left\{\left[\phi_{C}^{0}-\frac{m^{2}}{4 M_{Y} M_{N}} \phi_{C}^{1}\right]+\frac{m^{2}}{2 M_{Y} M_{N}} \phi_{S O}^{0} \mathbf{L} \cdot \mathbf{S}+\frac{m^{4}}{16 M_{Y}^{2} M_{N}^{2}} \frac{3}{(m r)^{2}} \phi_{T}^{0} Q_{12}\right\}\right] \mathcal{P}, \\
V_{S}^{n . l .}(r) & =\frac{m}{4 \pi}\left[\frac{1}{2} g_{13}^{S} g_{24}^{S} \phi_{C}^{0}\right] \mathcal{P} .
\end{aligned}
$$

(d) Axial-vector $K_{1 A^{-}}$meson exchange $J^{P C}=1^{++}$:

$$
\begin{aligned}
V_{A}(r)= & -\frac{m}{4 \pi}\left[\left\{g_{13}^{A} g_{24}^{A}\left(\phi_{C}^{0}+\frac{2 m^{2}}{3 M_{Y} M_{N}} \phi_{C}^{1}\right)+\frac{m^{2}}{6 M_{Y} M_{N}}\left(g_{13}^{A} f_{24}^{A}+f_{13}^{A} g_{24}^{A}\right) \frac{\sqrt{M_{Y} M_{N}}}{\mathcal{M}} \phi_{C}^{1}+f_{13}^{A} f_{24}^{A} \frac{m^{4}}{12 M_{Y} M_{N} \mathcal{M}^{2}} \phi_{C}^{2}\right\}\left(\sigma_{1} \cdot \boldsymbol{\sigma}_{2}\right)\right. \\
& \left.-\frac{m^{2}}{4 M_{Y} M_{N}}\left\{\left[g_{13}^{A} g_{24}^{A}-2\left(g_{13}^{A} f_{24}^{A}+f_{13}^{A} g_{24}^{A}\right) \frac{\sqrt{M_{Y} M_{N}}}{\mathcal{M}}\right] \phi_{T}^{0}-f_{13}^{A} f_{24}^{A} \frac{m^{2}}{2 \mathcal{M}^{2}} \phi_{T}^{1}\right\} S_{12}+\frac{m^{2}}{2 M_{Y} M_{N}} g_{13}^{A} g_{24}^{A} \phi_{S O}^{0} \mathbf{L} \cdot \mathbf{S}\right] \mathcal{P}
\end{aligned}
$$

$V_{A}^{n . l .}(r)=-\frac{m}{4 \pi}\left[\frac{3}{2} g_{13}^{A} g_{24}^{A} \phi_{C}^{0}\left(\sigma_{1} \cdot \sigma_{2}\right)\right] \mathcal{P}$.

(e) Axial-vector $K_{1 B^{-}}$meson exchange $J^{P C}=1^{+-}$:

$$
\begin{aligned}
V_{B}(r) & =-\frac{m}{4 \pi} \frac{4 M_{N} M_{Y}}{m^{2}}\left[f_{13}^{B} f_{24}^{B}\left\{\frac{m^{2}}{12 M_{Y} M_{N}}\left(\phi_{C}^{1}+\frac{m^{2}}{4 M_{Y} M_{N}} \phi_{C}^{2}\right) \sigma_{1} \cdot \sigma_{2}+\frac{m^{2}}{4 M_{Y} M_{N}}\left(\phi_{T}^{0}+\frac{m^{2}}{4 M_{Y} M_{N}} \phi_{T}^{1}\right) S_{12}\right\}\right] \mathcal{P}, \\
V_{B}^{n . l .}(r) & =-\frac{m}{4 \pi} \frac{3 M_{N} M_{Y}}{2 m^{2}}\left[f_{13}^{B} f_{24}^{B}\left(\frac{1}{3}\left(\sigma_{1} \cdot \sigma_{2}\right) \phi_{C}^{1}+S_{12} \phi_{T}^{0}\right)\right] \mathcal{P} .
\end{aligned}
$$

(f) Diffractive-exchange: Since in the ESC16-model the diffractive Pomeron and Odderon exchanges are SU(3) singlets, there are no contribution to $S \neq 0$ exchange potentials.

Above, in Eqs. (4.15)-(4.20), the exchange operator is defined as

$$
\mathcal{P}=-\mathcal{P}_{x} \mathcal{P}_{\sigma}
$$

where $\mathcal{P}_{x}$ and $\mathcal{P}_{\sigma}$ are the space- and spin-exchange operators respectively. The extra $\left(-\mathcal{P}_{\sigma}\right)$ operator in Eq. (4.16) for the antisymmetric spin-orbit potential is explained in Appendix D. We note that $-\mathcal{P}_{\sigma} \mathcal{P}=\mathcal{P}_{x}$, which is well defined for the coupled singlet-triplet systems.

\section{One-boson-exchange interactions in configuration space II}

Here we give the extra potentials due to the zeroes in the scalar and axial- $A$ vector form factors:

(a) Scalar mesons:

$$
\begin{gathered}
\Delta V_{S}(r)-\frac{m}{4 \pi} \frac{m^{2}}{U^{2}}\left[g _ { 1 3 } ^ { S } g _ { 2 4 } ^ { S } \left\{\left[\phi_{C}^{1}-\frac{m^{2}}{4 M_{Y} M_{N}} \phi_{C}^{2}\right]\right.\right. \\
\left.\left.+\frac{m^{2}}{2 M_{Y} M_{N}} \phi_{S O}^{1} \mathbf{L} \cdot \mathbf{S}+\frac{m^{4}}{16 M_{Y}^{2} M_{N}^{2}} \phi_{T}^{1} Q_{12}\right\}\right] \mathcal{P} .
\end{gathered}
$$

(b) Axial mesons: The extra contribution to the potentials coming from the zero in the axial-vector meson form factor are obtained from the expression (4.11) by 
making substitutions as follows:

$$
\begin{aligned}
& \Delta V_{A}^{(1)}(r) \\
& \quad=V_{A}^{(1)}\left(\phi_{C}^{0} \rightarrow \phi_{C}^{1}, \phi_{T}^{0} \rightarrow \phi_{T}^{1}, \phi_{S O}^{0} \rightarrow \phi_{S O}^{1}\right) \frac{m^{2}}{U^{2}} .
\end{aligned}
$$

Note that we do not include the similar $\Delta V_{A}^{(2)}(r)$ since they involve $\mathbf{k}^{4}$ terms in momentum space. Then,

$$
\begin{aligned}
V_{A}^{(1)}(r) & \\
= & -\frac{g_{13}^{A} g_{24}^{A}}{4 \pi} m\left[\phi_{C}^{0}\left(\sigma_{1} \cdot \sigma_{2}\right)-\frac{1}{12 M_{Y} M_{N}}\right. \\
& \times\left(\nabla^{2} \phi_{C}^{0}+\phi_{C}^{0} \nabla^{2}\right)\left(\sigma_{1} \cdot \boldsymbol{\sigma}_{2}\right) \\
& +\frac{3 m^{2}}{4 M_{Y} M_{N}} \phi_{T}^{0} S_{12}+\frac{m^{2}}{2 M_{Y} M_{N}} \phi_{S O}^{0} \mathbf{L} \cdot \mathbf{S} \\
& \left.+\frac{m^{2}}{4 M_{Y} M_{N}} \frac{M_{N}^{2}-M_{Y}^{2}}{M_{Y} M_{N}} \phi_{S O}^{(0)} \cdot \frac{1}{2}\left(\sigma_{1}-\sigma_{2}\right) \cdot \mathbf{L}\right] \mathcal{P} .
\end{aligned}
$$

\section{E. PS-PS-exchange interactions in configuration space}

In Figs. 3 and 4 of Ref. [2], the included double pseudoscalar (PS) exchange graphs are shown schematically. Explicit expressions for $K^{i r r}(\mathrm{BW})$ and $K^{i r r}(\mathrm{TMO})$ were derived [29], where also the terminology BW and TMO is explained. The TPS potentials for nucleon-nucleon have been given in detail in Ref. [30]. The generalization to baryon-baryon is similar to that for the OBE potentials. So, we substitute $M \rightarrow$ $\sqrt{M_{Y} M_{N}}$, and include all PS-PS possibilities with coupling constants as in the OBE potentials. As compared to nucleonnucleon in Ref. [30], we have here in addition the potentials with double $K$ exchange. The masses are the physical pseudoscalar meson masses. For the intermediate two-baryon states, we take into account the effects of the different thresholds. We have not included uncorrelated PS-vector, PS-scalar, or PS-diffractive exchange. This is because the range of these potentials is similar to that of the vector, scalar, and axialvector potentials. Moreover, for potentially large potentials, in particularly those with scalar mesons involved, there will be very strong cancellations between the planar and crossed-box contributions.

\section{F. MPE-exchange interactions}

In Fig. 4 of Ref. [1], the pair graphs are shown. In this work, we include only the one-pair graphs. The argument for neglecting the two-pair graph is to avoid some double counting. Viewing the pair vertex as containing heavy-meson exchange means that the contributions from $\rho(760)$ and $\epsilon=$ $f_{0}(620)$ to the two-pair graphs are already accounted for by our treatment of the broad $\rho$ and $\epsilon$ OBE potential. The MPE potentials for nucleon-nucleon have been given in Ref. [30]. The generalization to baryon-baryon is similar to that for the TPS potentials. For the intermediate two-baryon states, we neglect the effects of the different two-baryon thresholds. The inclusion of these, although in principle possible, would complicate the computation of the potentials considerably and the influence is not expected to be significant. The generalization of the pair couplings to baryon-baryon is described in Ref. [7], Sec. III. Also here in $Y N$, we have in addition to Ref. [30] included the pair potentials with $K K, K K^{*}$, and $K \kappa$ exchange. The convention for the MPE coupling constants is the same as in Ref. [30].

\section{G. Meson-pair potentials and axial pairs (second kind, $J^{P C}=1^{+-}$)}

Recently we have completed the $1 / M, 1 / M^{2}$ corrections to the adiabatic approximation for the pair potentials. The main reason is the need for a careful evaluation of the antisymmetric spin-orbit terms for $\Lambda N$, in particular for pair interactions involving strangeness exchange like $\pi-K, \pi-K^{*}$. From this evaluation, new contributions emerged, in particular for the axial pair interactions $J^{P C}=1^{++}, 1^{+-}$, leading to a substantial improvement with regard to the experimental spin-orbit splittings [16]. In our fitting procedure for the $Y N$ data, the spin orbit plays no role. However, also new $1 / M$ corrections for the spin-spin and tensor potentials were obtained for the axial-pair interaction of the second kind, i.e., $J^{P C}=1^{+-}$. These are relevant for the fits presented in this paper and will be given in this section. Below, we give the full one-pair exchange potential as used at present, because it has not been published before. In the ESC04 models, only the leading, i.e., the $(1 / M)^{0}$ terms, were used. For the derivation of the soft-core pair-interactions, we refer the reader to Ref. [30]. Below, we report on this derivation for the axial-pair terms of the second kind. The used pair interaction Hamiltonian for, e.g., the $(\pi \omega)$ pair is

$$
\mathcal{H}_{B}=g_{(\pi \omega)} \bar{\psi} \gamma_{5} \sigma_{\mu \nu} \tau \psi \cdot \partial^{\nu}\left(\pi \phi_{\omega}^{\mu}\right) /\left(m_{\pi} \mathcal{M}\right),
$$

which gives the $B B m_{1} m_{2}$ vertex

$$
\bar{u}\left(\mathbf{p}^{\prime}\right) \Gamma_{B}^{(2)} u(\mathbf{p})=i \frac{g_{(\pi \omega)_{1}}}{m_{\pi} \mathcal{M}}\left[\left( \pm \omega_{1} \pm \omega_{2}\right) \boldsymbol{\sigma} \cdot \boldsymbol{\omega}+\boldsymbol{\sigma} \cdot \mathbf{k} \omega^{0}\right] .
$$

The full SU(3) structure is given in Ref. [7], Sec. III A. It is assumed that this pair coupling is dominated by the SU(3)octet symmetric coupling and is given by the SU(3)-octet symmetric couplings Hamiltonian in terms of SU(2)-isospin invariants and $\mathrm{SU}(3)$ isoscalar factors:

$$
\begin{aligned}
\mathcal{H}_{B_{8} V P}= & \frac{g_{B_{8} V P}}{\sqrt{6}}\left\{\frac{1}{2}\left[\left(\mathbf{B}_{1}^{\mu} \cdot \boldsymbol{\rho}_{\mu}\right) \eta_{8}+\left(\mathbf{B}_{1}^{\mu} \cdot \boldsymbol{\pi}_{\mu}\right) \phi_{8}\right]\right. \\
& +\frac{\sqrt{3}}{4}\left[\mathbf{B}_{1} \cdot\left(K^{* \dagger} \boldsymbol{\tau} K\right)+\text { H.c. }\right] \\
& +\frac{\sqrt{3}}{4}\left[\left(K_{1}^{\dagger} \boldsymbol{\tau} K^{*}\right) \cdot \boldsymbol{\pi}+\left(K_{1}^{\dagger} \boldsymbol{\tau} K\right) \cdot \boldsymbol{\rho}+\text { H.c. }\right] \\
& -\frac{1}{4}\left[\left(K_{1}^{\dagger} \cdot K^{*}\right) \eta_{8}+\left(K_{1}^{\dagger} \cdot K\right) \phi_{8}+\text { H.c. }\right] \\
& \left.+\frac{1}{2} H^{0}\left[\boldsymbol{\rho} \cdot \boldsymbol{\pi}-\frac{1}{2}\left(K^{* \dagger} \cdot K+K^{\dagger} \cdot K^{*}\right)-\phi_{8} \eta_{8}\right]\right\} .
\end{aligned}
$$

Here, $\mathbf{B}_{1} \propto\left[\bar{\psi} \gamma_{5} \boldsymbol{\tau} \sigma_{\mu \nu} \psi\right]$. For a definition of the octet fields $\eta_{8}, \phi_{8}$ in terms of the physical mesons, see Ref. [7]. From the 
pair-interaction Hamiltonian (4.27), one can easily read off the different meson pairs that occur from the $J^{P C}=1^{+-}$vertex. In Appendix $\mathrm{C}$, we give the explicit potentials generated by the pair interaction (4.27).

\section{H. Treatment meson widths}

The treatment of the broad mesons $\rho$ and $\epsilon$ is as usual in the Nijmegen models. For the $\rho$ meson, the same parameters are used in the OBE models [12,13]. (In Appendix A, the procedure of the incorporation of the large widths is reviewed.) For the $\epsilon=f_{0}(620)$ assuming $m_{\epsilon}=620 \mathrm{MeV}$ and $\Gamma_{\epsilon}=464 \mathrm{MeV}$, we use the Bryan-Gersten dipole parametrization [31], corresponding to the pole $E(\varepsilon)=(552-i$ 232) $\mathrm{MeV}$ [32]. For the choosen mass and width, they are $m_{1}=455.15919 \mathrm{MeV}, m_{2}=1158.56219 \mathrm{MeV}$, and $\beta_{1}=$ $0.28193, \beta_{2}=0.71807$. The treatment of the scalar $\kappa(861)$ as a broad meson is new. From $m_{a_{0}}(962), m_{\epsilon}(620), m_{S^{*}}(993)$, and the scalar mixing $\theta_{S}\left(44^{0}\right)$, the GMO scheme [33] gives $m_{\kappa}=861$. With $\Gamma_{\kappa}=450 \mathrm{MeV}$ good results for $Y N$ are obtained. These values for the mass and width correspond closely to the pole $E(\kappa)=(826-i 449) \mathrm{MeV}$ found in the analysis of Ref. [34].

\section{SHORT-RANGE PHENOMENOLOGY}

It is well known that the most extensive study of the baryon-baryon interactions using meson exchange has difficulties achieving sufficiently repulsive short-range interactions in two channels, namely, (i) the $\Sigma^{+} p\left(I=3 / 2,{ }^{3} S_{1}\right)$ and (ii) the $\Sigma N\left(I=1 / 2,{ }^{1} S_{0}\right)$ channel. The short-range repulsion in baryon-baryon comes in principle from two sources: (a) meson and multigluon exchange, and (b) the occurrence of forbidden states by the Pauli principle, henceforth referred to as Pauli repulsion or quark core. As for (a) in the ESC model [6,7] the short-range repulsion comes from vector-meson exchange and Pomeron-Odderon exchange (i.e., multigluon). The possibility of mechanism (b) has been explored in the quark-cluster model. See the reviews in Refs. [19,20].

Analyzing the Pauli repulsion in terms of the $\mathrm{SU}(3, F)$ irreps, we find that the forbidden $L=0 B B$ states, which are classified in $\mathrm{SU}(6, F S)$ by the [51] irrep, indeed occur dominantly in the $\operatorname{SU}(3, F)$ irreps $\{10\}$ and $\left\{8_{s}\right\}$. These $\mathrm{SU}(3)$ irreps dominate the $\Sigma^{+} p\left(I=3 / 2,{ }^{3} S_{1}\right)$ and the $\Sigma N\left(I=1 / 2,{ }^{1} S_{0}\right)$ states respectively. These facts open the possibility to incorporate the exceptionally strong Pauli repulsion for these states by enhancing the Pomeron coupling in the ESC approach to baryon-baryon. For the other $B B$ states, the [51] irrep is present also, but roughly with an equal weight as the [33] irrep. Only in a few $S=-2$ channels, e.g., $\Xi N(I=1, S=0)$, there is a stronger presence of the irrep [51]. Therefore, a slightly moderated $\mathrm{SU}(3, F)$-singlet Pomeron exchange can effectively take care of this quark-core phenomenologically, together with multigluon-exchange effects.

\section{A. Relation $\mathrm{SU}(3, F)$-irreps and $\mathrm{SU}(6, F S)$-irreps classification $Y N$ states}

In Table I, the $\mathrm{SU}(3, F)$-contents of the $N N$ and $Y N$ states are shown. In Table II, we show the weights of the
TABLE I. SU $(3, F)$ contents of the various potentials on the isospin basis.

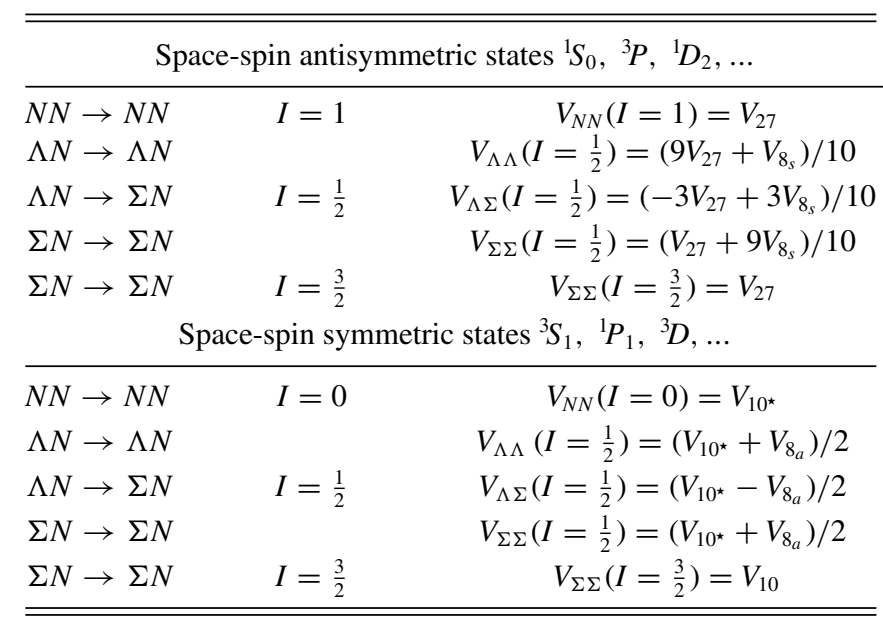

SU(6,FS) irreps. These are taken from Ref. [19], Table I, where the $\mathrm{SU}(6, F S)$ classifications are given. Analyzing now the $(\Lambda N, \Sigma N)$ system for $(S=0, I=1 / 2)$, we find from these tables

$$
\begin{aligned}
\left(\begin{array}{l}
V_{\Lambda N, \Lambda N} \\
V_{\Sigma N, \Sigma N}
\end{array}\right) & =\left(\begin{array}{cc}
\frac{1}{2} & \frac{1}{2} \\
\frac{17}{18} & \frac{1}{18}
\end{array}\right)\left(\begin{array}{l}
V_{[51]} \\
V_{[33]}
\end{array}\right) \\
& =\left(\begin{array}{cc}
\frac{9}{10} & \frac{1}{10} \\
\frac{1}{10} & \frac{9}{10}
\end{array}\right)\left(\begin{array}{l}
V_{\{27\}} \\
V_{\left\{8_{s}\right\}}
\end{array}\right) .
\end{aligned}
$$

(1) From (5.1), we obtain by simple matrix operations the relation between the $\mathrm{SU}(6, F S)$ irreps and the $\mathrm{SU}(3, F)$ irrreps, which read

$$
\left(\begin{array}{l}
V_{\{27\}} \\
V_{\left\{8_{s}\right\}}
\end{array}\right)=\left(\begin{array}{cc}
\frac{4}{9} & \frac{5}{9} \\
1 & 0
\end{array}\right)\left(\begin{array}{l}
V_{[51]} \\
V_{[33]}
\end{array}\right) .
$$

(2) Also, we can read off from the tables the following relations:

$$
\begin{aligned}
V_{N N}(I=1, S=0) & =\frac{4}{9} V_{[51]}+\frac{5}{9} V_{[33]}=V_{\{27\}} \\
V_{N N}(I=0, S=1) & =\frac{4}{9} V_{[51]}+\frac{5}{9} V_{[33]}=V_{\left\{10^{*}\right\}} \\
V_{\Lambda N}\left(I=\frac{1}{2}, S=1\right) & =\frac{1}{2} V_{[51]}+\frac{1}{2} V_{[33]}=\frac{1}{2} V_{\left\{10^{*}\right\}}+\frac{1}{2} V_{\left\{8_{a}\right\}}
\end{aligned}
$$

TABLE II. SU $(6, F S)$ contents of the various potentials on the spin-isospin basis.

\begin{tabular}{lcc}
\hline \hline & $(S, I)$ & $V=a V_{[51]}+b V_{[33]}$ \\
\hline$N N \rightarrow N N$ & $(0,1)$ & $V_{N N}=\frac{4}{9} V_{[51]}+\frac{5}{9} V_{[33]}$ \\
$N N \rightarrow N N$ & $(1,0)$ & $V_{N N}=\frac{4}{9} V_{[51]}+\frac{5}{9} V_{[33]}$ \\
$\Lambda N \rightarrow \Lambda N$ & $(0,1 / 2)$ & $V_{\Lambda \Lambda}=\frac{1}{2} V_{[51]}+\frac{1}{2} V_{[33]}$ \\
$\Lambda N \rightarrow \Lambda N$ & $(1,1 / 2)$ & $V_{\Lambda \Lambda}=\frac{1}{2} V_{[51]}+\frac{1}{2} V_{[33]}$ \\
$\Sigma N \rightarrow \Sigma N$ & $(0,1 / 2)$ & $V_{\Sigma \Sigma}=\frac{17}{18} V_{[51]}+\frac{1}{18} V_{[33]}$ \\
$\Sigma N \rightarrow \Sigma N$ & $(1,1 / 2)$ & $V_{\Sigma \Sigma}=\frac{1}{2} V_{[51]}+\frac{1}{2} V_{[33]}$ \\
$\Sigma N \rightarrow \Sigma N$ & $(0,3 / 2)$ & $V_{\Sigma \Sigma}=\frac{4}{9} V_{[51]}+\frac{5}{9} V_{[33]}$ \\
$\Sigma N \rightarrow \Sigma N$ & $(1,3 / 2)$ & $V_{\Sigma \Sigma}=\frac{8}{9} V_{[51]}+\frac{1}{9} V_{[33]}$ \\
\hline \hline
\end{tabular}


From these equations, we can solve the $\mathrm{SU}(3, F)$ irreps $\{27\},\left\{10^{*}\right\}$, and $\left\{8_{a}\right\}$ in terms of the $\mathrm{SU}(6, F S)$ irreps.

Listing the results, we now have

$$
\begin{aligned}
V_{\{27\}} & =\frac{4}{9} V_{[51]}+\frac{5}{9} V_{[33]}, \\
V_{\left\{10^{*}\right\}} & =\frac{4}{9} V_{[51]}+\frac{5}{9} V_{[33]}, \\
V_{\{10\}} & =\frac{8}{9} V_{[51]}+\frac{1}{9} V_{[33]}, \\
V_{\left\{8_{a}\right\}} & =\frac{5}{9} V_{[51]}+\frac{4}{9} V_{[33]}, \\
V_{\left\{8_{s}\right\}} & =V_{[51]} .
\end{aligned}
$$

We see from these results that the [51] irrep has a large weight in the $\{10\}$ and $\left\{8_{s}\right\}$ irrep, which gives an argument for the presence of a strong Pauli repulsion in these $\mathrm{SU}(3, F)$ irreps.

According to the study of the wide range of mesonexchange models in the past decade using the ESC approach, as a working hypothesis, we assume that the apparent lack of an exceptionally strong repulsion in the ESC model for the states in the $\mathrm{SU}(3, F)$ irreps $\{10\}$ and $\left\{8_{s}\right\}$ cannot be cured by meson exchange. The inclusion of this possible "forbidden state" effect can be done phenomenologically in the ESC approach by making an effective Pomeron potential as the sum of pure Pomeron exchange and of a Pomeron-like representation of the Pauli repulsion. As a consequence, the effective Pomeron potential gets quite strong in the $\mathrm{SU}(3, F)$ irreps $\{10\}$ and $\left\{8_{s}\right\}$. In this way, we incorporate the Pauli repulsion effect in the ESC approach in this paper.

\section{B. Parametrization quark-core effects}

Using a linear parametrization of the quark-core effects, we split the repulsive short-range Pomeron-like $N N$ potential as follows:

$$
\begin{aligned}
V_{P N N} & =\left(1-a_{\mathrm{PB}}\right) V_{P N N}+a_{\mathrm{PB}} V_{P N N} \\
& \equiv V_{N N}(\mathrm{POM})+V_{N N}(\mathrm{~PB}),
\end{aligned}
$$

where $V_{N N}(\mathrm{POM})$ represents the genuine Pomeron $N N$ potential and $V_{N N}(\mathrm{~PB})$ denotes the structural effects of the quark-core forbidden [51] configuration, i.e., a Pauli-blocking (PB) effect. So $a_{\mathrm{PB}}$ denotes the quark-core fraction of the total Pomeron-like potential. A similar relation holds for all $B B$ channels:

$$
\begin{aligned}
V_{P B B} & =\left(1-a_{\mathrm{PB}}\right) V_{P B B}+a_{\mathrm{PB}} V_{P B B} \\
& \equiv V_{B B}(\mathrm{POM})+V_{B B}(\mathrm{~PB}) .
\end{aligned}
$$

Since the Pomeron is a unitary singlet, its contribution is the same for all $B B$ channels (apart from some small baryon mass breaking effects). The PB effect for the $B B$ channels is assumed to be proportional to the relative weight of the forbidden [51] configuration compared to its weight in $N N$ :

$$
V_{B B}(\mathrm{~PB})=\left(w_{B B}[51] / w_{N N}[51]\right) V_{N N}(\mathrm{~PB}) .
$$

Then, we have

$$
V_{P B B}=\left(1-a_{\mathrm{PB}}\right) V_{P N N}+a_{\mathrm{PB}}\left(\frac{w_{B B}[51]}{w_{N N}[51]}\right) V_{P N N} .
$$

TABLE III. Effective Pomeron+PB contribution on the spin and isospin basis.

\begin{tabular}{lccc}
\hline \hline & $(S, I)$ & $V_{P B B} / V_{P N N}$ & ESC16 \\
\hline$N N \rightarrow N N$ & $(0,1)$ & 1 & 1.000 \\
$N N \rightarrow N N$ & $(1,0)$ & 1 & 1.000 \\
$\Lambda N \rightarrow \Lambda N$ & $(0,1 / 2)$ & $1+\frac{1}{8} a_{\mathrm{PB}}$ & 1.049 \\
$\Lambda N \rightarrow \Lambda N$ & $(1,1 / 2)$ & $1+\frac{1}{8} a_{\mathrm{PB}}$ & 1.049 \\
$\Sigma N \rightarrow \Sigma N$ & $(0,1 / 2)$ & $1+\frac{9}{8} a_{\mathrm{PB}}$ & 1.439 \\
$\Sigma N \rightarrow \Sigma N$ & $(1,1 / 2)$ & $1+\frac{1}{8} a_{\mathrm{PB}}$ & 1.049 \\
$\Sigma N \rightarrow \Sigma N$ & $(0,3 / 2)$ & 1 & 1.000 \\
$\Sigma N \rightarrow \Sigma N$ & $(1,3 / 2)$ & $1+a_{\mathrm{PB}}$ & 1.390 \\
\hline \hline
\end{tabular}

For example, in the SU(3) irrep $\{10\}$, e.g., the $\Sigma^{+} p\left({ }^{3} S_{1}, T=\right.$ $3 / 2$ ) channel, one has $w_{B B}[51]=8 / 9=2 w_{N N}[51]$ and therefore $V_{10}(\mathrm{~PB})=2 V_{N N}(\mathrm{~PB})$. Consequently, the total shortrange repulsive potential, i.e., the effective Pomeron, becomes $V_{10}=\left(1-a_{\mathrm{PB}}\right) V_{P N N}+2 a_{\mathrm{PB}} V_{P N N}=\left(1+a_{\mathrm{PB}}\right) V_{P N N}$. In Table III, we give the factors for the various $S=0$ and $S=-1$ $B B$ channels as well as the results in the ESC16 model.

In principle, one might choose a different mass for the quark-core repulsive potential. However, this extra parameter does not lead to better fits to $N N$ and/or $Y N$. Therefore, we keep for the Pauli blocking the same mass as the Pomeron mass. The value of the PB factor $a_{\mathrm{PB}}$ is searched in the fit to the $Y N$ data. The $S=-2$ PB effects are then also entirely determined. In the case of the models ESC08 $\mathrm{a}^{\prime}$ and ESC08b', only the channels where $w_{B B}[51]$ is conspicuously large are treated approximately this way, but with equal weights. These channels are $\Sigma^{+} p\left({ }^{3} S_{1}, T=3 / 2\right), \Sigma N\left({ }^{1} S_{0}, T=1 / 2\right)$, and $\Xi N\left({ }^{1} S_{0}, T=1\right)$. A subtle treatment of all $B B$ channels according to this linear scheme is characteristic of the ESC16 model. The parameter $a_{\mathrm{PB}}$ turns out to be about $39 \%$. This means that the quark-core repulsion is roughly $64 \%$ of the genuine Pomeron repulsion. Around $r=0$, the quark-core repulsion comes out at about $76 \mathrm{MeV}$, whereas the pure Pomeron repulsion is $118 \mathrm{MeV}$. The $\chi_{Y N}^{2}$ is not very sensitive to $a_{\mathrm{PB}}$. For $a_{\mathrm{PB}}=0.29,0.34,0,39,0.44$, we get $\chi_{Y N}^{2}=50.0,51.5,54.2,57.8$, respectively. The choice $a_{\mathrm{PB}}=$ 0.39 is a compromise between the $\chi_{Y N}^{2}$ and an unattractive two-body $U_{\Sigma}$.

\section{ESC16: FITTING $N N \oplus Y N \oplus Y Y$ DATA}

In this section, we describe mainly the recent changes in the fitting process. For details on the standard $N N \oplus Y N$ fitting, we refer readers to Ref. [14].

(i) As usual, we fit to the 1993 Nijmegen representation of the $\chi^{2}$ hypersurface of the $N N$ scattering data below $T_{\text {lab }}=350 \mathrm{MeV}[35,36]$. The $N N$ low-energy parameters are fitted along with the scattering data. In order to accommodate the differences between the ${ }^{1} S_{0}$ waves for $p p, n p$, and $n n$ in the model, we introduce some charge independence breaking by taking different electric $\rho$ couplings $g_{p p \rho} \neq g_{n p \rho} \neq g_{n n \rho}$, where $g_{n n \rho}$ is considered to be the SU(3)-octet coupling. With this phenomenological device, we fit the difference 
TABLE IV. ESC16 SU(3) coupling constants, $F /(F+D)$ ratios, and mixing angles. The values with asterisks $(*)$ have are theoretical input or determined by the fitting and the constraint from the $Y N$ analysis.

\begin{tabular}{lcrrcl}
\hline \hline Mesons & & $\{1\}$ & $\{8\}$ & $\mathrm{F} /(\mathrm{F}+\mathrm{D})$ & Angles $(\mathrm{deg})$ \\
\hline Pseudoscalar & $\mathrm{f}$ & 0.3389 & 0.2684 & $\alpha_{P}=0.365^{*}$ & $\theta_{P}=-11.4^{*}$ \\
Vector & $\mathrm{g}$ & 3.1983 & 0.5793 & $\alpha_{V}^{e}=1.0^{*}$ & $\theta_{V}=39.1^{*}$ \\
& $\mathrm{f}$ & -2.2644 & 3.7791 & $\alpha_{V}^{m}=0.4655$ & \\
Axial(A) & $\mathrm{g}$ & -0.8826 & -0.8172 & $\alpha_{A}=0.3830$ & $\theta_{A}=50.0^{*}$ \\
& $\mathrm{f}$ & -6.2681 & -1.6521 & $\alpha_{A}^{p}=0.3830^{*}$ & \\
Axial(B) & $\mathrm{f}$ & -0.9635 & -2.2598 & $\alpha_{B}=0.4^{*}$ & $\theta_{B}=35.26^{*}$ \\
Scalar & $\mathrm{g}$ & 3.2369 & 0.5393 & $\alpha_{S}=1.0^{*}$ & $\theta_{S}=44.0^{*}$ \\
Diffractive & $g_{P}$ & 2.7191 & & & $a_{\mathrm{PB}}=0.39$ \\
& $g_{O}$ & 4.1637 & & & \\
& $f_{O}$ & -3.8859 & & & \\
\hline \hline
\end{tabular}

between the ${ }^{1} S_{0}(p p)$ and ${ }^{1} S_{0}(n p)$ phases, and the different $N N$ scattering lengths and effective ranges very well. We have found $g_{p p \rho}=0.5932, g_{n p \rho}=0.5427$, which are not far from $g_{n n \rho}=0.5793$ (cf. Table IV).

(ii) Simultaneously we fit to $52 Y N$ scattering data. These data consist of the usual set of 35 low-energy $Y N$ data, as used in Refs. $[7,13,14]$, plus three total $\Sigma^{+} p$ cross sections from the recent KEK experiment E289 [37] and some $\Lambda p$ elastic and inelastic data [38] and $\Sigma^{-} p$ elastic data at higher energies [39]. For the total $\Sigma^{+} p$ and $\Sigma^{-} p$ elastic cross sections, we have performed the same redefinition as Eq. (6.3) of Ref. [14].

\section{A. Incorporation hypernuclei information}

A novel feature in the simultaneous fitting procedure is the inclusion of constraints from information derived from hypernuclei and hypernuclear matter. For the $\Lambda N$ interaction, this means not only (a) the usual absence of bound states but also (b) the requirement of a sizable spin splitting, leading to $U_{\sigma \sigma}>1$ (cf. Sec. IX A). (c) Because of the experimental absence of $\Sigma$ hypernuclei, we require the total single-particle $\Sigma$ potential in nuclear matter $U_{\Sigma}$ to be overall repulsive. In the $S=-2$ channels, there are two clear experimental indications: (d) from the analysis of the Nagara event [40] of the double- $\Lambda$ hypernucleus ${ }_{\Lambda \Lambda}^{6} \mathrm{He}$ it appears that the forces in the $\Lambda \Lambda\left({ }^{1} S_{0}\right)$ channel are weakly attractive, indicated by a scattering length $a_{\Lambda \Lambda}\left({ }^{1} S_{0}\right) \sim-(0.5-1.0) \mathrm{fm}$ [41]. This evidence has been incorporated in the fit in the form of pseudodata for the $\left({ }^{1} S_{0}\right) \Lambda \Lambda$ scattering length $a_{\Lambda \Lambda}=-0.8 \pm(0.2-0.4)$, with the error depending on the desired impact in the fitting process. (e) Experimentally, the $\Xi$-nucleus interaction seems to be attractive from analyses of events with twin- $\Lambda$ hypernuclei in emulsion data, where the initial $\Xi^{-}$energies were determined after $\Xi^{-} p-\Lambda \Lambda$ conversion in nuclei. The $\Xi$ nucleus interaction can be described well with a Wood-Saxon potential with a depth of $\approx 14 \mathrm{MeV}$ [42]. On the other hand, the $\Xi^{-} p$ scattering seems experimentally too small to support a sizable $U_{\Xi}$ well depth. (In Ref. [5], the tensor interaction from the pairs was enlarged, giving a large well depth having a $\Xi N\left({ }^{3} S_{1}, T=1\right)$ bound state, but this gives cross sections that are too large.) Details will be presented in a forthcoming paper on the strangeness $S=-2$ channels. The fit has resulted in an excellent simultaneous $N N \oplus Y N \oplus Y Y$ fit. We obtained for the $N N$ data $\chi^{2} / N N_{\text {data }}=1.10$ with also very good results for the $N N$ low-energy parameters: the deuteron binding energy and the $p p, n p$, and $n n$ scattering lengths and effective ranges. For the $Y N$ data, $\chi^{2} / Y N_{\text {data }}=1.04$. The ESC16 fits were achieved with only physical meson-coupling parameters, which are partial-wave independent. The quality of the $N N$ fit is at par with models of Reid-like potentials like the Nijm93, Nijm I, and Nijm II, which are effective $N N$ potentials with some meson parameters adjusted per partial wave [43,44]. Since the ESC16 model is an extension of the ESC04 model, it is not surprising that in the simultaneous $N N, Y N$, and $Y Y$ fit the OBE couplings could be kept in line with the naive predictions of the QPC model $[6,10]$. Just as in ESC04, most of the $F /(F+D)$ ratios are fixed by QPC, for both the OBE and MPE couplings. Once more, we stress the fact that in the simultaneous fit of the $N N, Y N$, and $Y Y$ data, a single set of parameters was used. Of course, the accurate and very numerous $N N$ data put strong constraints on the parameters. However, the $Y N$ data, plus the constraints for the $Y N$ and $Y Y$ channels, are also quite relevant for the set of parameters finally obtained. In particular, certain fitted $F /(F+D)$ ratios are obviously influenced by the $Y N$ data.

\section{B. Coupling parameters and $N N \oplus Y N \oplus Y Y$ fit}

For the diffractive $0^{++}$exchanges, we restrict ourselves to the SU(3)-singlet part, henceforth referred to as "Pomeron." The possible $J=0$ part of the tensor-meson exchange $[12,13]$ is not considered. The mass parameter of the Pomeron is fitted to be $m_{P}=212.05 \mathrm{MeV}$. The diffractive $1^{--}$exchange Odderon is also an $\mathrm{SU}(3)$ singlet with a fitted mass $m_{O}=$ $268.82 \mathrm{MeV}$.

Summarizing the fitted parameters in ESC16, we have the following:

(1) Meson couplings: $f_{N N \pi}, f_{N N \eta^{\prime}}, g_{N N \rho}, g_{N N \omega}, f_{N N \rho}$, $f_{N N \omega}, g_{N N a_{0}}, g_{N N \epsilon}, \quad g_{N N a_{1}}, f_{N N a_{1}}, g_{N N f_{1}^{\prime}}, f_{N N f_{1}^{\prime}}, f_{N N b_{1}}$, $f_{N N h_{1}^{\prime}}$.

(2) Pair couplings: $g_{N N(\pi \pi)_{1}}, f_{N N(\pi \pi)_{1}}, g_{N N(\pi \rho)_{1}}, g_{N N \pi \omega}$, $g_{N N \pi \eta}, g_{N N \pi \epsilon}$.

(3) Diffractive couplings and masses: $g_{N N P}, g_{N N O}$, $f_{N N O}, a_{\mathrm{PB}}, m_{P}, m_{O}$.

(4) $F /(F+D)$ ratios: $\alpha_{V}^{m}, \alpha_{A}$.

(5) Cutoff masses: $\Lambda_{8}^{P}=\Lambda_{1}^{P}=\Lambda_{8}^{B}=\Lambda_{1}^{B}, \Lambda_{8}^{V}, \Lambda_{1}^{V}, \Lambda_{8}^{S}$, $\Lambda_{1}^{S}, \Lambda_{8}^{A}=\Lambda_{1}^{A}$.

These are in total 34 physical parameters: (i) 14 meson couplings, (ii) $2 F /(F+D)$ ratios, (iii) 4 diffractive couplings and 2 mass parameters, (iv) 6 meson-pair couplings, and (v) 6 cutoff mass parameters.

As compared to the ESC04 model, we have added in ESC16 the following fitting parameters: (i) the derivative axial couplings $f_{N N a_{1}}, f_{N N f_{1}^{\prime}}$, (ii) the $1^{+-}$axial couplings $f_{N N b_{1}}, f_{N N h_{1}^{\prime}}$, (iii) the Odderon couplings $g_{N N O}, f_{N N O}$, and mass $m_{O}$, and (iv) the Pomeron Pauli-blocking parameter $a_{\mathrm{PB}}$, i.e., 8 new physical parameters. All new parameters have been explained above. They introduce new dynamical refinements and effects into the model, which have resulted in a quality 
TABLE V. Coupling constants for model ESC16, divided by $\sqrt{4 \pi} . M$ refers to the meson. The coupling constants are listed in the order pseudoscalar, vector $(g$ and $f$ ), axial vector $\mathrm{A}(g$ and $f)$, scalar, axial vector $\mathrm{B}$, and diffractive.

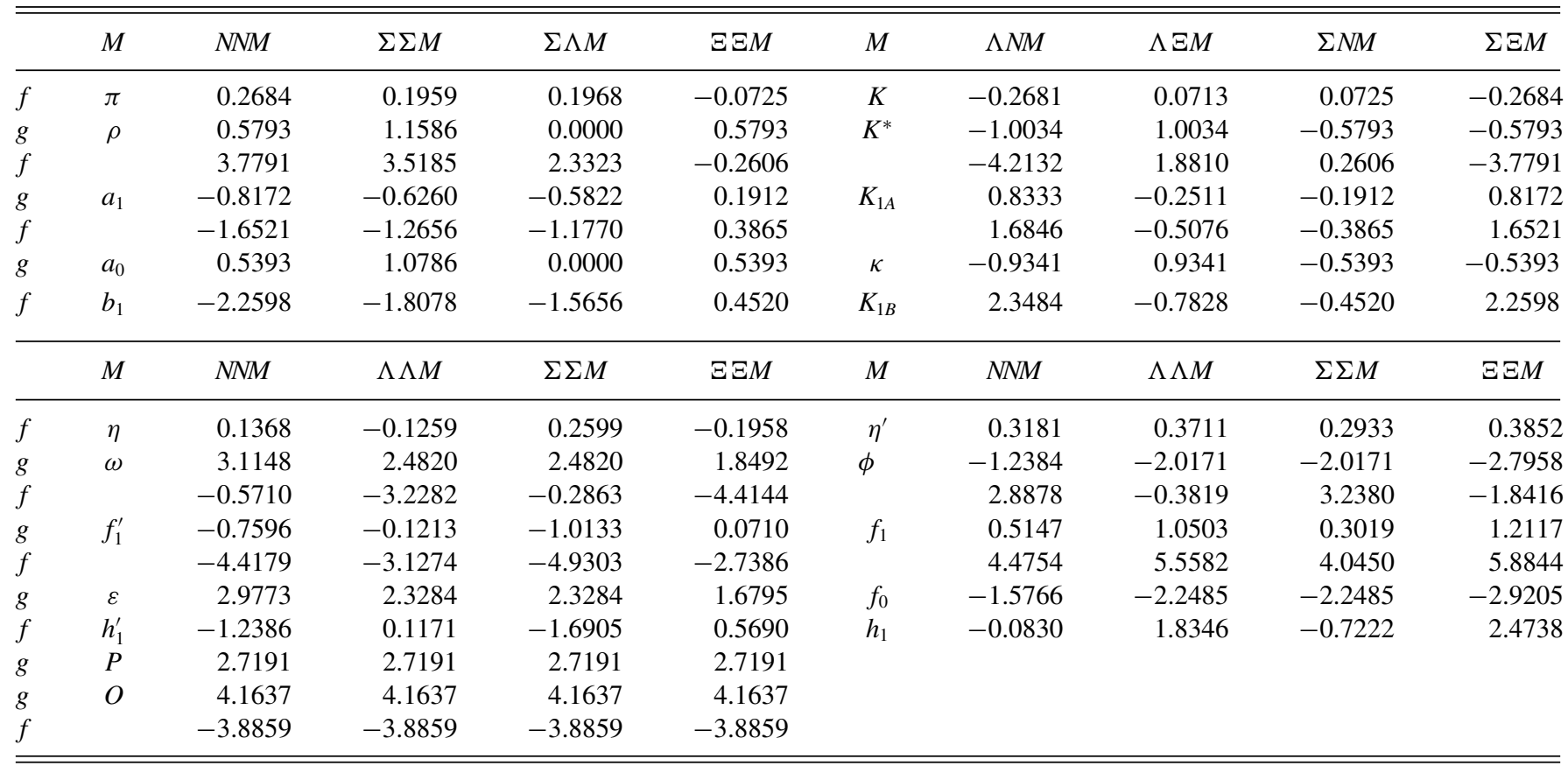

of the combined $N N \oplus Y N \oplus Y Y$ fit for the $N N$ phases equal to those of a purely $N N$ fit. Some other parameters have been set, e.g., many $F /(F+D)$ ratios (see below) and a few cutoff parameters.

The pair coupling $g_{N N(\pi \pi)_{0}}$ is set to be zero, which is motivated in the Nijmegen soft-core models in view of the fact that in $\pi N$ it is constrained by chiral symmetry. In the fitting process, we look for solutions which have meson couplings which are reasonably close to the naive predictions of the QPC model. This is also the case for the $F /(F+D)$ ratios, both for meson and for pair couplings. During the fitting, we experienced a rather shallow dependence on the $F /(F+D)$ ratio $\alpha^{P}$ for the pseudoscalar octet. In fact, we could obtain very good $Y N$ and $N N$ fits in a values range $0.33-0.40$. Therefore, we have fixed it at the value $\alpha_{P}=0.365$ obtained from the Cabibbo theory of semileptonic decay of baryons [45]. Furthermore, the meson-pair couplings turn out to come out rather close to predictions based on the heavymeson-saturation model. So, the fit parameters are (i) physical parameters, i.e., they can be checked in other reactions, and (ii) many are constraints in the QPC model.

In this work, like in the ESC04 models [6,7], the form factors depend on the SU(3) assignment of the mesons, In principle, we introduce form factor masses $\Lambda_{8}$ and $\Lambda_{1}$ for the $\{8\}$ and $\{1\}$ members of each meson nonet, respectively. Moreover, for the $I=0$ mesons, we assign the $\{1\}$ cutoff to the dominant singlet meson and the $\{8\}$ cutoff to the dominant octet meson, as if there were no meson mixing. For example, we assign $\Lambda_{1}$ to $\eta^{\prime}, \omega, \epsilon$, and $\Lambda_{8}$ to $\eta, \phi, S^{*}$, etc. Notice that the strange octet-mesons $K$ are given the same $\{8\}$ form factors as their nonstrange companions. For the cutoff masses $\Lambda$, we used as free search parameters $\Lambda_{8}^{P}=\Lambda_{1}^{P}$ for the pseudoscalar mesons, $\Lambda_{8}^{V}$ and $\Lambda_{1}^{V}$ for the vector mesons, and $\Lambda_{8}^{S}$ and $\Lambda_{1}^{S}$ for the scalar mesons. Furthermore, we finally used $\Lambda_{8}^{A}=\Lambda_{1}^{A}$ for the axial mesons with $J^{P C}=1^{++}$. For the axial mesons with $J^{P C}=1^{+-}$(B mesons), the cutoff masses have been set equal to those of the pseudoscalar mesons $\Lambda_{8}^{B}=\Lambda_{8}^{P}$ and $\Lambda_{1}^{B}=\Lambda_{1}^{P}$. Some of the previous $\{8\}$ and $\{1\}$ form factors have been chosen to be equal as a consequence of the impossibility to distinguish them in the fitting process.

Similar to ESC04, we introduce a zero in the form factors of mesons, which are $P$-wave bound states in a $q \bar{q}$ picture. These are the scalar mesons $\left({ }^{3} P_{0}\right)$ and the axial vector $\left({ }^{3} P_{1}\right)$ mesons. Like in ESC04, we use a fixed zero by taking $U=$ $750 \mathrm{MeV}$ in Eqs. (4.22) and (4.24).

\section{COUPLING CONSTANTS, $F /(F+D)$ RATIOS, AND MIXING ANGLES}

Like in ESC04, we constrained the OBE couplings by the naive predictions of the QPC model [9]. We kept during the searches all OBE couplings in the neighborhood of these predictions but less tight than in ESC04. The same holds for the searched $\alpha=F /(F+D)$ ratios, i.e., for the BBM couplings and the $B B$-pair couplings. In fact, only two meson-coupling $F /(F+D)$ ratios were allowed to vary during the searches: $\alpha_{V}^{m}$ for the vector mesons and $\alpha_{A}$ for the axial-vector mesons. As mentioned above, $\alpha_{P}$ was kept to the value $\alpha_{P}=0.365$. Furthermore, we kept $\alpha_{V}^{E}=1$ as in all our previous work and also $\alpha_{S}=1.0$, i.e., equal to the QPC value. Furthermore, $\alpha_{B}=0.4$. For the fitted ESC16 $N N$ meson couplings and cutoff masses we refer to Table III of Ref. [1].

The mixing angles for the various meson nonets are discussed in Ref. [1]. The used values can be found in Table IV. Here we discuss only aspects specific for the $Y N$ channels. In Table IV, the ESC16 SU(3) singlet and octet couplings 
TABLE VI. Pair-meson coupling constants employed in the ESC16 MPE potentials. Coupling constants are at $\mathbf{k}^{2}=0$. The $F /(F+D)$ ratio are QPC predictions, except that $\alpha_{(\pi \omega)}=\alpha_{P}$, which is very close to $\mathrm{QPC}$.

\begin{tabular}{lccrc}
\hline \hline$J^{P C}$ & SU(3) irrep & $(\alpha \beta)$ & $g / 4 \pi$ & $F /(F+D)$ \\
\hline $0^{++}$ & $\{1\}$ & $g(\pi \pi)_{0}$ & & \\
$0^{++}$ & $\{1\}$ & $g(\sigma \sigma)$ & & \\
$0^{++}$ & $\{8\}_{s}$ & $g(\pi \eta)$ & -0.6894 & 1.000 \\
$1^{--}$ & $\{8\}_{a}$ & $g(\pi \pi)_{1}$ & 0.2519 & 1.000 \\
& & $f(\pi \pi)_{1}$ & -1.7762 & 0.400 \\
$1^{++}$ & $\{8\}_{a}$ & $g(\pi \rho)_{1}$ & 5.7017 & 0.400 \\
$1^{++}$ & $\{8\}_{a}$ & $g(\pi \sigma)$ & -0.3899 & 0.400 \\
$1^{++}$ & $\{8\}_{a}$ & $g(\pi P)$ & & \\
$1^{+-}$ & $\{8\}_{s}$ & $g(\pi \omega)$ & -0.3287 & 0.365 \\
\hline \hline
\end{tabular}

$g / \sqrt{4 \pi}$, the $F /(F+D)$ ratios, and the used mixing angles are listed.

\section{A. Coupling and SU(3) MPE parameters}

In Table $\mathrm{V}$, we list the couplings of the physical mesons to the nucleons $(Y=1)$ and to the hyperons with $Y=0$ or $Y=-1$. These were calculated using unbroken SU(3) symmetry. Next to the values in the table, we have incorporated, like in the ESC04 model [7], charge symmetry breaking (CSB) between $\Lambda p$ and $\Lambda n$ with nonzero $\Lambda$ couplings of the $I=1$ mesons and $I=1$ pairs due to $\Lambda-\Sigma^{0}$ mixing.

In Table VI, we present the fitted pair couplings for the MPE potentials. We recall that only one-pair graphs are included, in order to avoid double counting; see Ref. [1]. The $F /(F+D)$ ratios are all fixed, assuming heavy-boson dominance of the pair vertices. The ratios are taken from the QPC model for $q \bar{q}$ systems with the same quantum numbers as the dominating boson. Only the ratio in the system with the pseudoscalar quantum numbers deviates slightly from QPC, since it has been set equal to the value of $\alpha_{P}=0.365$. The BB-pair couplings are calculated, assuming unbroken SU(3) symmetry, from the $N N$-pair coupling and the $F /(F+D)$ ratio using $\mathrm{SU}(3)$.

Unlike in Ref. [30], we did not fix pair couplings using a theoretical model, based on heavy-meson saturation and chiral symmetry. So, in addition to the 14 coupling parameters used in Ref. [30], we now have six pair-coupling fit parameters. In Table VI, the fitted pair couplings are given, and Appendix B gives the SU(3) structure of the pair couplings. As noted in Ref. [1], the $(\pi \pi)$ coupling gets a nonzero contribution from the $\left\{8_{s}\right\}$ pairs, giving $g_{(\pi \pi)_{0}}=-0.69 / 2=-0.35$, which is opposite to that of Ref. [30]. Also, the $f_{(\pi \pi)_{1}}$ pair coupling has an opposite sign as compared to Ref. [30]. In a model with a more complex and realistic meson dynamics [46], this coupling is predicted as found in the present ESC fit. The $(\pi \rho)_{1}$ coupling agrees nicely with $A_{1}$ saturation; see Ref. [30]. The pair couplings are used in a phenomelogical way in the ESC approach. They are in general not yet quantitatively understood and certainly deserve more study in the future.

The ESC model described here is fully consistent with SU(3) symmetry using a straightforward extension of the $N N$ model to $Y N$ and $Y Y$. For example, $g_{(\pi \rho)_{1}}=g_{A_{8} V P}$, and besides
TABLE VII. $U_{\Sigma}$ in $\mathrm{MeV}$ and $Y N \chi^{2}$ as a function of $a_{\mathrm{PB}}$.

\begin{tabular}{lcccc}
\hline \hline$a_{\mathrm{PB}}$ & $U_{\Sigma}$ & $\chi_{\Lambda p}^{2}$ & $\chi_{\Sigma^{+} p}^{2}$ & $\chi_{Y N}^{2}$ \\
\hline 0.29 & -7.9 & 10.0 & 14.7 & 50.0 \\
0.34 & -5.5 & 10.4 & 15.9 & 51.5 \\
0.39 & -3.3 & 11.6 & 17.3 & 54.2 \\
0.44 & -1.1 & 13.8 & 18.7 & 57.8 \\
0.49 & +0.8 & 16.7 & 20.4 & 61.6 \\
\hline \hline
\end{tabular}

$(\pi \rho)$ pairs one sees also that $K K^{*}(I=1)$ and $K K^{*}(I=0)$ pairs contribute to the $N N$ potentials. All $F /(F+D)$ ratios are taken fixed with heavy-meson saturation in mind. The approximation we have made in this paper is to neglect the baryon mass differences; i.e., we put $m_{\Lambda}=m_{\Sigma}=m_{N}$ in the calculation of the MPE potentials. This because we have not yet worked out the formulas for the inclusion of these mass differences, which is straightforward in principle.

\section{B. Parameters and hyperon-nucleon fit}

In this paper, all "best" low-energy $Y N$ data are included in the fitting. This is a selected set of 35 low-energy $Y N$ data, the same set has been used in Refs. [13,14]. To these, we added (i) 3 total $\Sigma^{+} p$ cross sections from the KEK experiment E289 [37], (ii) 7 elastic and 4 inelastic $\Lambda p$ cross sections from Berkeley [38], and (iii) 3 elastic $\Sigma^{-} p$ cross sections [39]. In Sec. VIII, all these data are presented together with the results. Next to these, we added pseudodata for the $\Lambda p$ scattering lengths in order to ensure that the $\Lambda p\left({ }^{1} S_{0}\right)$ forces are stronger than the $\Lambda p\left({ }^{3} S_{1}\right)$. Technically favored values of the $s$-wave scattering lengths for $\Lambda N$ were imposed as pseudodata during the fitting procedures, in order to get a proper spin splitting for the $\Lambda N$ interaction in hypernuclei. In nuclear matter, this would imply $U_{\sigma \sigma}>1$, where

$$
U_{\sigma \sigma}=\left[U_{\Lambda}\left({ }^{3} S_{1}\right)-3 U_{\Lambda}\left({ }^{1} S_{0}\right)\right] / 12 .
$$

In ESC16, with the treatment of the broad $\kappa(861)$ the $S$-wave scattering lenghts have become about equal $a_{s} \approx a_{t}$, leading to $U_{\sigma \sigma}<1$.

We added pseudodata for the $\Sigma^{+} p\left({ }^{3} S_{1}\right)$ scattering length with the goal to reach enough repulsion in this wave in order to have $U_{\Sigma}>0$. For the pseudodata in the $S=-2$ channels, we refer to Sec. VI. In the final stages of the fitting process, all pseudodata were turned off. In fm,

$$
\begin{gathered}
\hat{a}_{\Lambda p}\left({ }^{1} S_{0}\right)=-2.60 \pm(0.10-0.20), \\
\hat{a}_{\Lambda p}\left({ }^{3} S_{1}\right)=-1.60 \pm(0.10-0.20), \\
\hat{a}_{\Sigma^{+} p}\left({ }^{3} S_{1}\right)=+0.65 \pm(0.10-0.20),
\end{gathered}
$$

Also, during the fitting process, checks were done to prevent the occurrence of bound $\Lambda p$ states. Parameters, typically strongly influenced by the $Y N$ data, are as follows:

(1) $F /(F+D)$ parameters: $\alpha_{V}^{m}$ and to a less extent $\alpha_{A}$. For the sensitivity of $\alpha_{P}$, see Sec. VII.

(2) Pauli-blocking fraction parameter $a_{\mathrm{PB}}$.

The dependence of $a_{\mathrm{PB}}$ in the fit to $Y N$ and $Y Y$ is rather shallow in a range $0.20-0.40$. In Table VII, the dependence of 

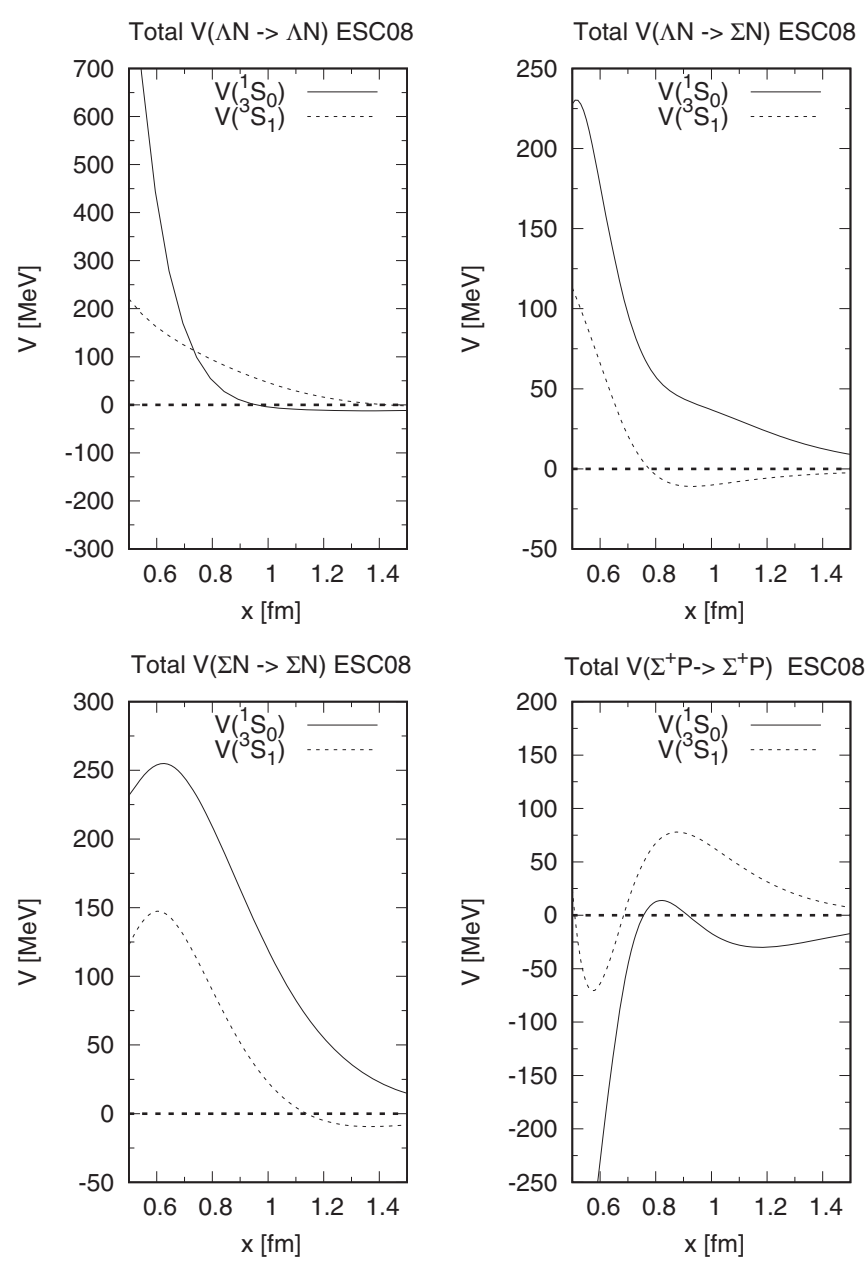

FIG. 1. Total potentials in the partial waves ${ }^{1} S_{0}$ and ${ }^{3} S_{1}$, for $I=$ $1 / 2$ and $I=3 / 2$ states.

the $\chi^{2}$ of the $52 Y N$ data, and the $U_{\Sigma}$ is illustrated. A minimal value for $\chi^{2}$ leads to $a_{\mathrm{PB}}=0.29$, whereas a positive value of $U_{\Sigma}$ requires $a_{\mathrm{PB}}>0.49$. As a compromise, we have chosen $a_{\mathrm{PB}}=0.39$.

Since all couplings and SU(3) parameters are completely fixed, the $S=-2$ (and $-3,-4$ ) results of ESC16 are completely determined. Finally, we want to mention that in the fitting process we have, if necessary, accounted for the vast difference in quality of the data. The abundance of the 4313 precise $N N$ data is to be contrasted to the 52 less precise $Y N$ data. In the simultaneous fit, we require for both the $N N$ and for the $Y N$ that the quality of the partial fit is comparable, i.e., $\chi^{2} / N N_{\text {data }} \approx \chi^{2} / Y N_{\text {data }}$. If necessary, we add weight factors to the partial sums in the total $\chi^{2}$. It turned out that in the last stages of the fitting process, the weight factors are equal.

\section{Hyperon-nucleon potentials}

In this section, we use FORTRAN code ESC2016/ HNPOTESC16 [47]. In Fig. 1, we plot the total potentials for the $S$-wave channels $\Lambda N \rightarrow \Lambda N, \Lambda N \rightarrow \Sigma N$, and $\Sigma N \rightarrow \Sigma N$. Note for the soft-core model the typical structure of the $\Sigma^{+} p\left({ }^{3} S_{1}\right)$ potential. Most contributions to the spin-spin potentials are proportional to $\mathbf{k}^{2}$, and hence have zero volume integral. This causes the attraction in the inner region.

Figures for the OBE, TME, and MPE contributions are similar to those for the ESC04 model and have been displayed in Ref. [7]; we refer the interested reader to this reference and likewise for the contributions of the various types of mesons to the OBE potentials and the different kinds of pair potentials to MPE.

\section{ESC16-MODEL AND YN RESULTS}

\section{A. Hyperon-nucleon $(S=-1)$ cross sections and phases}

The used $Y N$ scattering data from Refs. [48-53] in the combined $N N$ and $Y N$ fit are shown in Table VIII. The $N N$ interactions put very strong constraints on most of the parameters, and so we are left with only a limited set of parameters which have some freedom to steer the $Y N$ channels as compared to the $N N$ channels.

The aim of the present study was to construct a realistic potential model for baryon-baryon systems with parameters that are optimal theoretically but at the same time describe the baryon-baryon scattering data very satisfactorily.

This model can then be used with a great deal of confidence in calculations of hypernuclei and in their predictions for the $S=-2,-3$, and -4 sectors. Especially for the latter application, these models will be the first models for the $S=-2,-3,-4$ sectors to have their theoretical foundation in the $N N$ and $Y N$ sectors.

The $\chi^{2}$ on the $52 Y N$ scattering data for the ESC16 model is given in Table VIII. The $\Lambda N$ total cross sections have been calculated with $L \leqslant 2$ and the $\Sigma N$ total cross sections with $L \leqslant 1$. For the definition of the capture ratio at rest, given in the last row of the table, see, e.g., Ref. [14]. This capture ratio turns out to be rather constant in the momentum range from 100 to $170 \mathrm{MeV} / c$. Obviously, for very low momenta, the cross sections are almost completely dominated by $s$ waves, and so the capture ratio in flight converges to the capture ratio at rest. For more details on the evaluation of these observables, we refer to earlier Nijmegen work on this subject, see e.g. [25].

The $\Sigma^{+} p$ nuclear-bar phase shifts as a function of energy are given in Table IX. Notice that the ${ }^{3} S_{1}$ phase shows repulsion.

The $\Lambda N$ nuclear-bar phase shifts as a function of energy are given in Table X. In Fig. 2, the $\Lambda p$ total cross sections are shown for ESC16 together with the data. At the $\Sigma N$ threshold, the cross section shows a sizable cusp with a large $D$-wave nuclear-bar phase shift $\delta\left({ }^{3} D_{1}\right)=69.10^{\circ}$. This signals the fact that in the $\Sigma N\left({ }^{3} S_{1}, I=1 / 2\right)$ state there is a strong attraction, with presumably a deuteron-like virtual bound state on the unphysical sheet. Also, in Fig. 2 we show the cross sections in the effective range approximation, dashed lines I and II. Line II is including the shape parameter in the effective range expansion. The two-term effective range expansion with the $a$ and $r$ parameters describes the $s$-wave phases well up to $p_{\Lambda} \approx 400 \mathrm{MeV} / \mathrm{c}$.

In Table XI, the low-energy parameters for $\Lambda p$ and $\Lambda n$ are shown. The singlet and triplet parameters are displayed with the $\Lambda \Sigma^{0}$ mixing turned on for pseudoscalar, vector, scalar, 
TABLE VIII. Comparison of the calculated ESC16 and experimental values for the $52 Y N$ data that were included in the fit. The superscipts $R H$ and $M$ denote, respectively, the Rehovoth-Heidelberg [48] and Maryland data [49]. Also included are (i) $3 \Sigma^{+} p$ cross sections at $p_{\text {lab }}=$ 400, 500, $650 \mathrm{MeV}$ from Ref. [37], (ii) $\Lambda p$ cross sections from Ref. [38]: 7 elastic between $350 \leqslant p_{\text {lab }} \leqslant 950$ and 4 inelastic with $p_{\text {lab }}=$ $667,750,850,950 \mathrm{MeV}$, and (iii) 3 elastic $\Sigma^{-} p$ cross sections at $p_{\text {lab }}=450,550,650 \mathrm{MeV}$ from Ref. [39]. The laboratory momenta are in $\mathrm{MeV} / \mathrm{c}$, and the total cross sections in $\mathrm{mb}$. The total $\chi^{2}=54.2$.

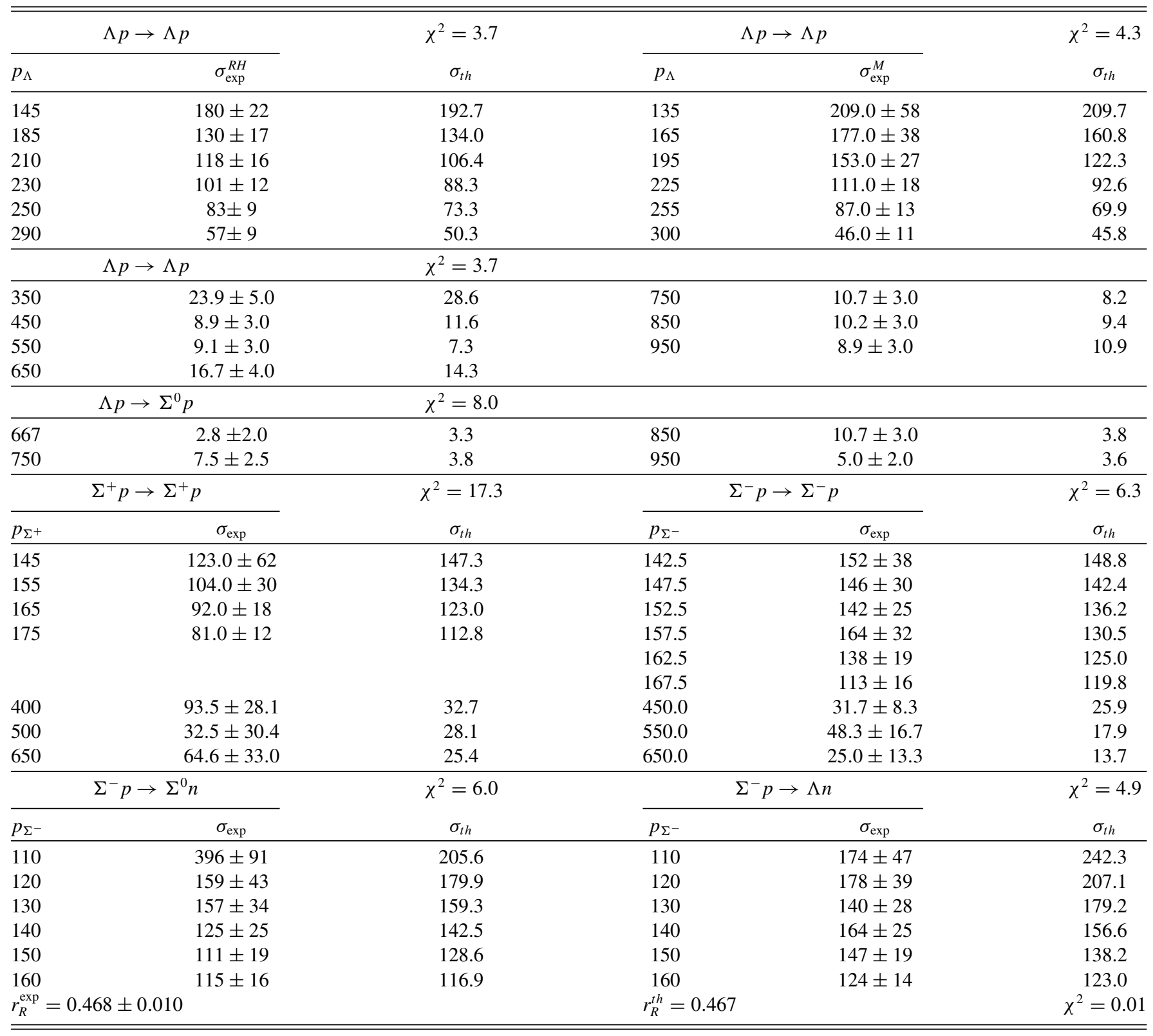

and meson pairs-, and ps-ps exchanges. Notice that the effect for the scalar mesons of the $\Lambda \Sigma^{0}$ mixing is zero because $\alpha_{s}=1.0$. It is clear from these tables that the total effect of the $\Lambda \Sigma^{0}$ mixing is about given by pseudoscalar and vector exchanges. The differences in the scattering lengths are

$$
\begin{aligned}
& \Delta a_{s}=a_{s}(\Lambda p)-a_{s}(\Lambda n)=+0.08 \mathrm{fm} \\
& \Delta a_{t}=a_{t}(\Lambda p)-a_{t}(\Lambda n)=-0.04 \mathrm{fm}
\end{aligned}
$$

These differences are comparable to those for the softcore OBE models $[13,14]$ and therefore predict a too small binding energy difference in the $A=4$ hypernuclei, which is $\Delta B_{\Lambda}(\exp )=B_{\Lambda}\left({ }_{\Lambda}^{4} \mathrm{He}\right)-B_{\Lambda}\left({ }_{\Lambda}^{4} \mathrm{H}\right)=(0.29 \pm 0.06) \mathrm{MeV}$. This in contrast to the hard-core (HC) model D, which has a much larger $\Delta a_{t}$ [24]. It appeared that CSB via meson mixing, like $\pi^{0}-\eta, \rho^{0}-\omega$, etc., is small and does not improve the CSB for ESC16, which is understandable in view of the large cancellations. However, as a consequence of the ESC models, there is a three-body force produced by the MPE interactions, which are fixed by the $B B$ fit. Therefore, the CSB in the $\Lambda N N$ potential may improve the CSB predictions significantly.

In Table XII, we list the $\Sigma^{+} p$ scattering lengths and effective ranges. Here, $\left(a_{s}, r_{s}\right)$ are these quantities for $\Sigma^{+} p\left({ }^{1} S_{0}\right)$ and $\left(a_{t}, r_{t}\right)$ for $\Sigma^{+} p\left({ }^{3} S_{1}\right)$. 
TABLE IX. ESC16 nuclear-bar $\Sigma^{+} p$ phases in degrees.

\begin{tabular}{|c|c|c|c|c|c|c|c|c|c|c|}
\hline$p_{\Sigma^{+}}$ & 100 & 200 & 300 & 400 & 500 & 600 & 700 & 800 & 900 & 1000 \\
\hline$T_{\mathrm{lab}}$ & 4.2 & 16.7 & 37.3 & 65.5 & 100.8 & 142.8 & 190.7 & 244.0 & 302.1 & 364.5 \\
\hline${ }^{1} S_{0}$ & 35.10 & 41.06 & 35.49 & 27.54 & 19.12 & 10.81 & 2.80 & -4.80 & -11.99 & -18.76 \\
\hline$\epsilon_{1}$ & -0.37 & -1.81 & -3.33 & -4.43 & -5.04 & -5.24 & -5.14 & -4.86 & -4.48 & -4.07 \\
\hline${ }^{3} P_{0}$ & 0.96 & 4.90 & 8.42 & 9.12 & 7.19 & 3.59 & -0.92 & -5.81 & -10.81 & -15.73 \\
\hline${ }^{1} P_{1}$ & 0.43 & 2.33 & 4.84 & 7.05 & 8.33 & 8.45 & 7.46 & 5.55 & 2.94 & -0.17 \\
\hline$\epsilon_{2}$ & -0.03 & -0.37 & -1.05 & -1.81 & -2.44 & -2.85 & -3.02 & -2.98 & -2.78 & -2.49 \\
\hline${ }^{3} D_{1}$ & 0.02 & 0.30 & 0.84 & 1.32 & 1.35 & 0.69 & -0.70 & -2.75 & -5.29 & -8.21 \\
\hline${ }^{1} D_{2}$ & 0.02 & 0.31 & 0.97 & 2.00 & 3.38 & 4.99 & 6.61 & 8.01 & 8.97 & 9.33 \\
\hline${ }^{3} D_{2}$ & -0.03 & -0.45 & -1.30 & -2.35 & -3.53 & -4.87 & -6.42 & -8.15 & -10.04 & -12.04 \\
\hline${ }^{3} D_{3}$ & 0.00 & 0.05 & 0.26 & 0.66 & 1.14 & 1.59 & 1.93 & 2.20 & 2.45 & 2.70 \\
\hline
\end{tabular}

Notice that the difference between $a_{s}$ in ESC08a" and ESC16. This is mainly a consequence of the inclusion of the nonlocal tensor force in $\Sigma^{+} p$ like in $p p$. This means that there is still less room for variations in the ${ }^{3} S_{1}$ wave because of the cross-section fit. Because in $\mathrm{SU}(3)$ the ${ }^{1} S_{0}$ wave is strongly constrained by $p p$, since the ${ }^{1} S_{0}$ states in $N N$ and $\Sigma^{+} p$ are both in the $\{27\}$ irrep. Therefore, much extra repulsion in the triplet wave is impossible.

In Figs. 3 and 4, the elastic and inelastic cross sections are shown, respectively.

\section{B. Potentials in SU(3) irreps}

In Fig. 5, the potentials $V_{\{\mu\}}[\mathrm{GeV}]$ in the $\mathrm{SU}(3)$ representations for $B B$ channels are shown. The solid curves are ESC16 including SU(3) breaking and the dashed ones are the SU(3)symmetric curves. In the latter, average masses are used, for the baryons the $\Lambda$ mass, and for the SU(3) nonets $400 \mathrm{MeV}$ for the pseudoscalar and $800 \mathrm{MeV}$ for the vector, scalar, axialvector nonets. The cutoff masses for pseudoscalar, vector, and axial vector have been set equal to the octet ones, i.e., $\Lambda_{1}^{P}=\Lambda_{*}^{P}$, etc. For the scalar nonet, we have taken $\Lambda_{8}^{S}=\Lambda_{1}^{S}$.

TABLE X. ESC16 nuclear-bar $\Lambda p$ phases in degrees.

\begin{tabular}{lrrrrrrr}
\hline \hline$p_{\Lambda}$ & 100 & 200 & 300 & 400 & 500 & 600 & \multicolumn{1}{c}{633.0} \\
\hline$T_{\text {lab }}$ & \multicolumn{1}{c}{4.5} & 17.8 & \multicolumn{1}{c}{39.6} & \multicolumn{1}{c}{69.5} & 106.9 & 151.1 & 167.3 \\
${ }^{1} S_{0}$ & 20.21 & 25.86 & 23.13 & 17.24 & 10.34 & 3.40 & 1.43 \\
${ }^{3} S_{1}$ & 20.25 & 26.62 & 24.84 & 19.99 & 14.17 & 8.51 & 7.46 \\
$\epsilon_{1}$ & 0.04 & 0.16 & 0.23 & 0.21 & 0.29 & 2.01 & 9.16 \\
${ }^{3} P_{0}$ & 0.02 & 0.08 & -0.19 & -1.34 & -3.54 & -6.51 & -7.52 \\
${ }^{1} P_{1}$ & -0.07 & -0.55 & -1.78 & -3.88 & -6.67 & -9.80 & -10.82 \\
${ }^{3} P_{1}$ & 0.00 & -0.10 & -0.60 & -1.70 & -3.25 & -4.67 & -4.71 \\
${ }^{3} P_{2}$ & 0.11 & 0.70 & 1.74 & 2.86 & 3.73 & 4.25 & 4.34 \\
$\epsilon_{2}$ & 0.00 & -0.00 & -0.04 & -0.15 & -0.31 & -0.50 & -0.57 \\
${ }^{3} D_{1}$ & 0.00 & 0.07 & 0.48 & 1.82 & 5.42 & 18.93 & 59.97 \\
${ }^{1} D_{2}$ & 0.00 & 0.06 & 0.37 & 1.12 & 2.36 & 3.96 & 4.53 \\
${ }^{3} D_{2}$ & 0.00 & 0.08 & 0.42 & 1.17 & 2.31 & 3.68 & 4.16 \\
${ }^{3} D_{3}$ & 0.00 & 0.05 & 0.27 & 0.76 & 1.52 & 2.41 & 2.71 \\
\hline \hline
\end{tabular}

Figure 5 shows that, in particular for $r \leqslant 0.5 \mathrm{fm}$, the SU(3) breaking is rather large.

The curves resemble qualitatively those obtained in lattice QCD, except for the $\{1\}$ irrep [54]. In the ESC model, the

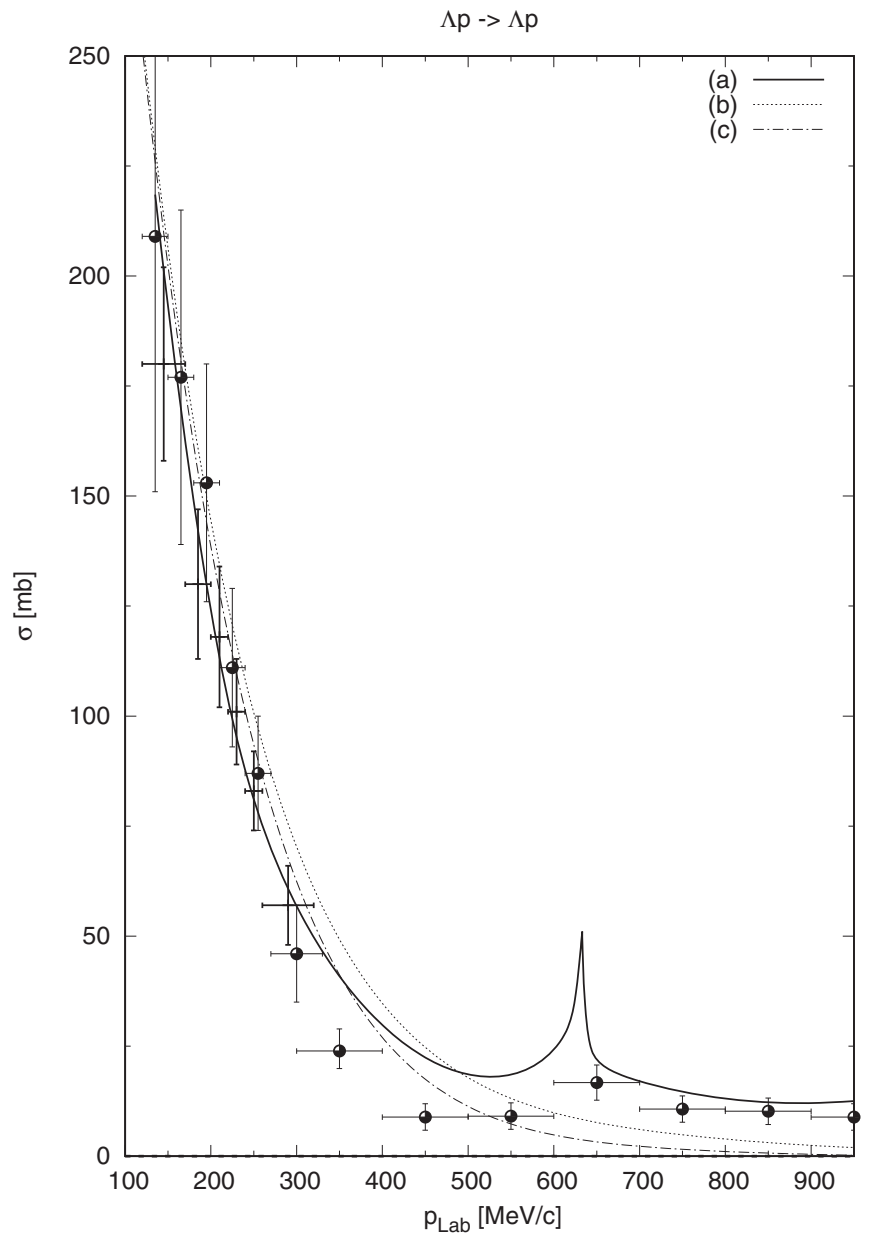

FIG. 2. Model fits total cross sections $\Lambda p$, and RehovothHeidelberg, Maryland, and Berkeley data. (a) ESC16. [(b), (c)] Effective range approximations I and II. 
TABLE XI. Comparison $\Lambda p$ and $\Lambda n$ scattering lengths and effective ranges in fm for different Nijmegen models.

\begin{tabular}{|c|c|c|c|c|c|c|c|c|}
\hline \multirow[t]{2}{*}{ Model } & \multicolumn{2}{|c|}{$\Lambda p$} & \multicolumn{2}{|c|}{$\Lambda n$} & \multicolumn{2}{|c|}{$\Lambda p$} & \multicolumn{2}{|c|}{$\Lambda n$} \\
\hline & $a_{s}$ & $a_{t}$ & $a_{s}$ & $a_{t}$ & $r_{s}$ & $r_{t}$ & $r_{s}$ & $r_{t}$ \\
\hline ESC16 & -1.88 & -1.86 & -1.96 & -1.84 & 3.58 & 3.37 & 3.65 & 3.33 \\
\hline NSC97e & -2.10 & -1.86 & -2.24 & -1.83 & 3.19 & 3.19 & 3.24 & 3.14 \\
\hline NSC97f & -2.51 & -1.75 & -2.68 & -1.67 & 3.03 & 3.32 & 3.07 & 3.34 \\
\hline NSC89 & -2.73 & -1.48 & -2.86 & -1.24 & 2.87 & 3.04 & 2.91 & 3.33 \\
\hline HC-D & -1.77 & -2.06 & -2.03 & -1.84 & 3.78 & 3.18 & 3.66 & 3.32 \\
\hline
\end{tabular}

behavior is typical for potentials with a strong spin-spin part, because the spin-spin potentials from pseudoscalar and vector exchange have zero volume integral forcing them to change sign for $r \approx 0.5 \mathrm{fm}$.

The similarity between the meson exchange and QCDlattice potentials shows that with the ESC realization of the program starting from the nuclear force, using $\mathrm{SU}(3, F)$ symmetry and the QM, a realistic generalization to the $B B$ force is achieved.

\section{ANALYSES WITH $G$-MATRIX INTERACTIONS}

The $G$-matrix theory gives a good starting point for studies of hyperonic many-body systems on the basis of free-space $Y N$ interaction models [55-57]. Here, the correlations induced by hyperonic coupling interactions such as $\Lambda N-\Sigma N$ ones are renormalized into single-channel $G$ matrices. These $G$-matrix interactions are considered as effective interactions used in models of hypernuclei. Thus, the hypernuclear phenomena and the underlying $Y N$ interaction models are linked through the $Y N G$-matrix interactions, and the hypernuclear information gives feedback to the interaction models. Here, the properties of $\Lambda N$ and $\Sigma N$ sectors of ESC16 in nuclear medium are studied on the basis of the $G$-matrix theory.

In Refs. [58-60], the three-body interaction is added on ESC16, composed of the multipomeron exchange repulsive potential (MPP) and the phenomenological three-baryon attraction (TBA). The effective two-body potential derived from MPP is given as

$V_{\mathrm{MPP}}(r ; \rho)=g_{P}^{(3)}\left(g_{P}\right)^{3} \frac{\rho}{\mathcal{M}^{5}} \frac{1}{4 \pi} \frac{4}{\sqrt{\pi}}\left(\frac{m_{P}}{\sqrt{2}}\right)^{3} \exp \left(-\frac{1}{2} m_{P}^{2} r^{2}\right)$,

where $g_{P}^{(3)}$ is the triple-Pomeron coupling, and the Pomeron mass $m_{P}$ and the two-body Pomeron coupling $g_{P}$ are fitted to the $N N$ data. In a similar way, one can obtain an effective two-body potential with a quartic Pomeron coupling $g_{P}^{(4)}$. TBA

TABLE XII. $\Sigma^{+} p$ scattering lengths and effective ranges in $\mathrm{fm}$.

\begin{tabular}{lcccc}
\hline \hline Model & $a_{s}$ & $a_{t}$ & $r_{s}$ & $r_{t}$ \\
\hline ESC16 & -4.30 & +0.57 & 3.25 & -3.11 \\
ESC08a" & -3.85 & +0.62 & 3.40 & -2.13 \\
ESC04d & -3.43 & +0.217 & 3.98 & -28.94 \\
\hline \hline
\end{tabular}
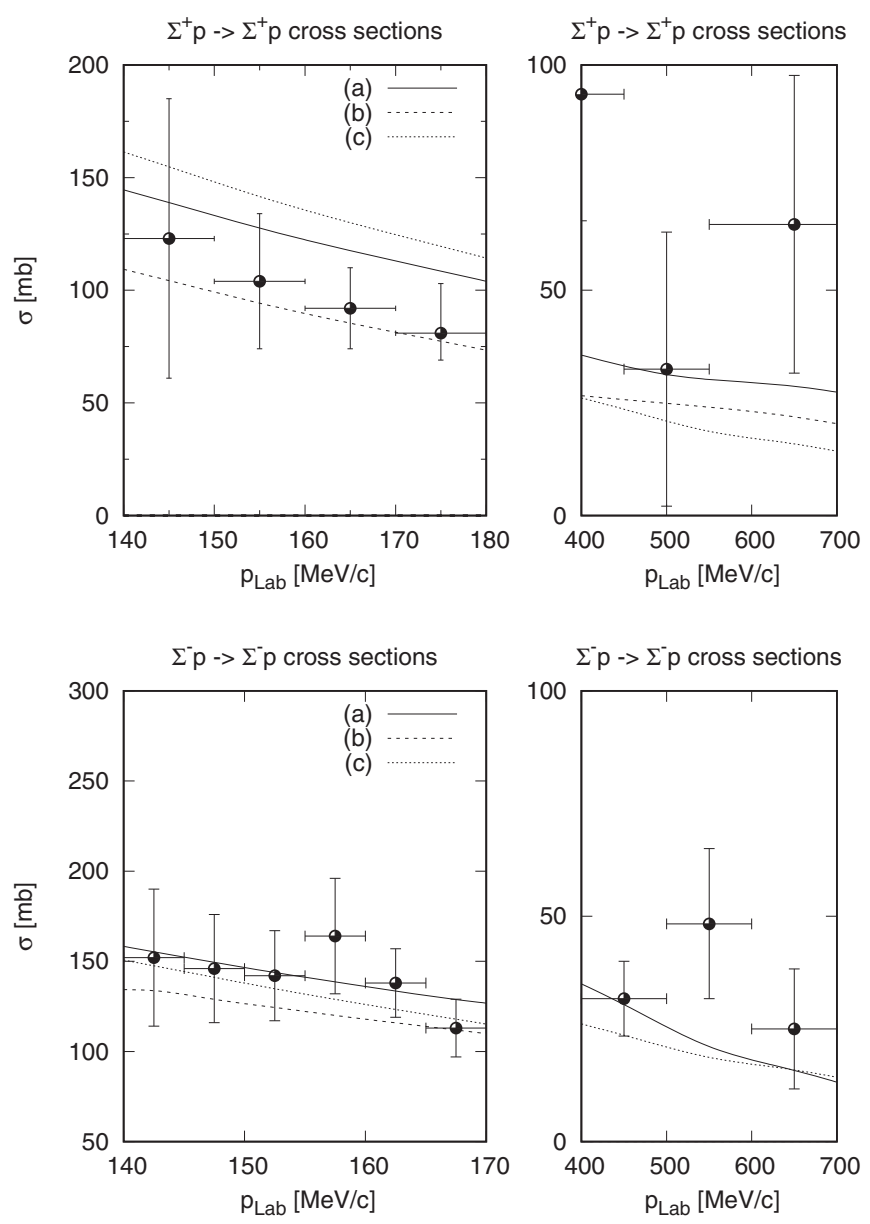

FIG. 3. Model fits total elastic cross sections $\Sigma^{ \pm} p$ and RehovothHeidelberg and KEK data. Left panels (a) ESC16, (b) ESC04d, and (c) NSC89. Right panels are the same.

also is given by a density-dependent two-body potential

$V_{\mathrm{TBA}}(r ; \rho)=V_{\mathrm{TBA}}^{0} \exp \left[-(r / 2.0)^{2}\right] \rho \exp (-\eta \rho)\left(1+P_{r}\right) / 2$,

$P_{r}$ being a space-exchange operator. The values of $g_{P}^{(3)}, g_{P}^{(4)}$, $V_{\mathrm{TBA}}^{0}$, and $\eta$ in $N N$ channels are adjusted to reproduce the angular distribution of ${ }^{16} \mathrm{O}+{ }^{16} \mathrm{O}$ elastic scattering at $E / A=$ $70 \mathrm{MeV}$ with use of the $G$-matrix folding potential, and values of the saturation density and the energy per nucleon there in nuclear matter [58-60]. We adopt here the parameter set $\left(g_{P}^{(3)}=5.25, g_{P}^{(4)}=87.0\right)$ that gives rise to the stiff equation of state of neutron matter with a maximum mass $2 M_{\odot}$ for a neutron star [58,59]. Other sets, like $\mathrm{MPa}$ and $\mathrm{MPa}^{+}$in Ref. [60], lead to similar results in the normal density region; differences appear only in the high-density region.

MPP works universally in all baryon-baryon channels according to its definition. Assuming here that TBA works also in $\Lambda N$ channels, the parameters are adjusted to reproduce well the energy spectra of $\Lambda$ hypernuclei. We take $V_{\mathrm{TBA}}^{0}=$ $-16.0 \mathrm{MeV}$, being more attractive than $V_{\mathrm{TBA}}^{0}=-8.0 \mathrm{MeV}$ in $N N$ channels, and the same value of $\eta=4.0 \mathrm{fm}^{3}$ for 

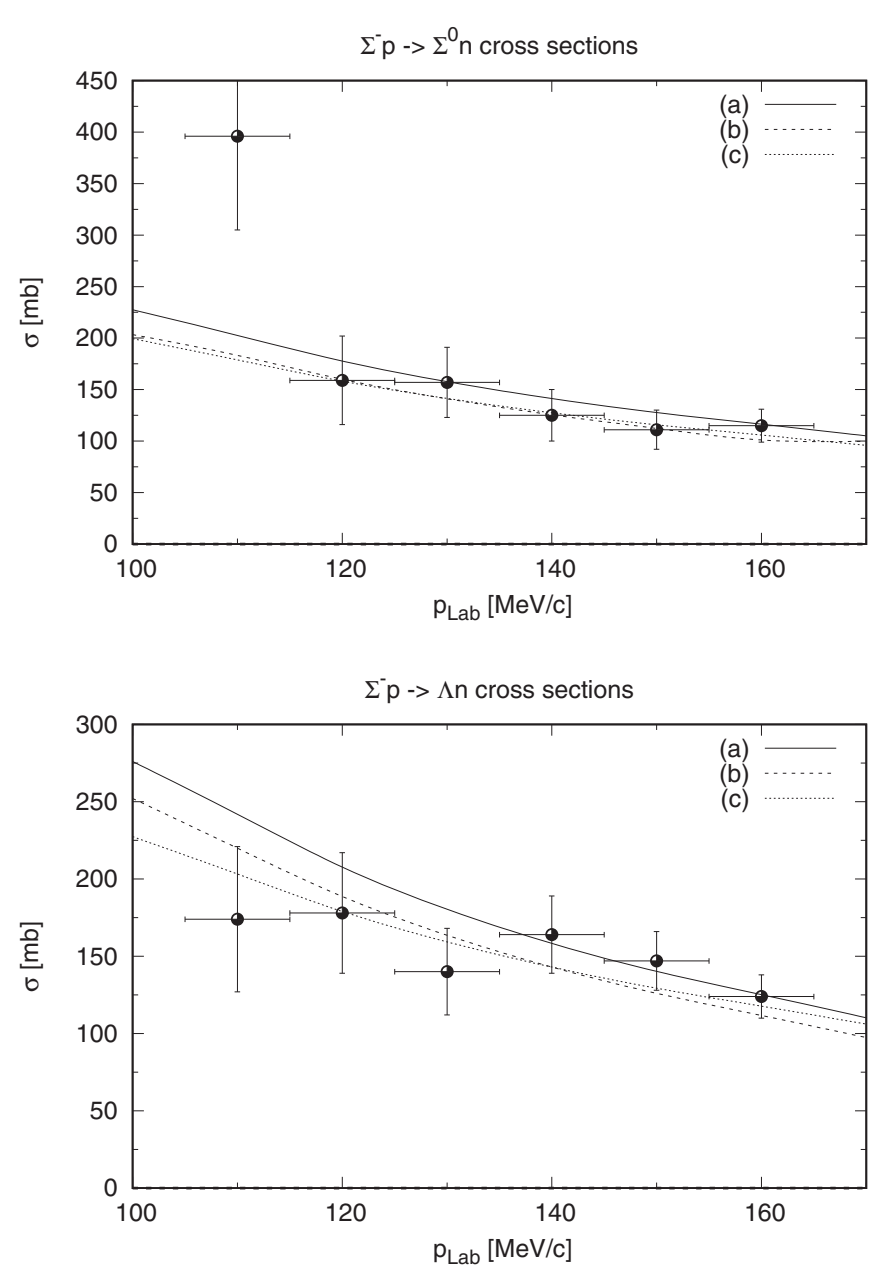

FIG. 4. Model fits total inelastic cross sections $\Sigma^{-} p \rightarrow$ $\Sigma^{0} n, \Lambda n$, and Rehovoth-Heidelberg data. (a) ESC16, (b) ESC04d, and (c) NSC89. Right panels are the same.

simplicity. Hereafter, the interaction ESC16+MPP+TBA is denoted as $\mathrm{ESC} 16^{+}$.

We start from the channel-coupled $G$-matrix equation for the baryon pair $B_{1} B_{2}$ in nuclear matter [55], where $B_{1} B_{2}=$ $\Lambda N$ and $\Sigma N$,

$$
G_{c c_{0}}=v_{c c_{0}}+\sum_{c^{\prime}} v_{c c^{\prime}} \frac{Q_{y^{\prime}}}{\omega-\epsilon_{B_{1}^{\prime}}-\epsilon_{B_{2}^{\prime}}+\Delta_{y y^{\prime}}} G_{c^{\prime} c_{0}},
$$

where $c$ denotes a $Y N$ relative state $(y, T, L, S, J)$ with $y=$ $\left(B_{1}, B_{2}\right) . S$ and $T$ are spin and isospin quantum numbers, respectively. Orbital and total angular momenta are denoted by $L$ and $J$, respectively, with $\mathbf{J}=\mathbf{L}+\mathbf{S}$. Then, a two-particle state is represented as ${ }^{2 S+1} L_{J}$. In Eq. (9.3), $\omega$ gives the starting energy in the starting channel $c_{0} . \Delta_{y y^{\prime}}=M_{B_{1}}+M_{B_{2}}-$ $M_{B_{1}^{\prime}}-M_{B_{2}^{\prime}}$ denotes the mass difference between two-baryon channels. The Pauli operator $Q_{y}$ acts on intermediate nucleon states in a channel $y=\left(B_{1}, B_{2}\right)=(\Lambda N, \Sigma N)$. We adopt here the continuous (CON) choice for intermediate single particle potentials in the $G$-matrix equation. The $G$-matrix equation (9.3) is represented in the coordinate space, whose solutions give $G$-matrix interactions. The hyperon single particle (s.p.) energy $\epsilon_{Y}$ in nuclear matter is given by

$$
\epsilon_{Y}\left(k_{Y}\right)=\frac{\hbar^{2} k_{Y}^{2}}{2 M_{Y}}+U_{Y}\left(k_{Y}\right),
$$

where $k_{Y}$ is the hyperon momentum. The potential energy $U_{Y}$ is obtained self-consistently in terms of the $G$ matrix as

$$
U_{Y}\left(k_{Y}\right)=\sum_{\left|\mathbf{k}_{N}\right|}\left\langle\mathbf{k}_{Y} \mathbf{k}_{N}\left|G_{Y N}\left(\omega=\epsilon_{Y}\left(k_{Y}\right)+\epsilon_{N}\left(k_{N}\right)\right)\right| \mathbf{k}_{Y} \mathbf{k}_{N}\right\rangle .
$$

\section{A. $\Lambda N$ G matrix}

Let us calculate $\Lambda$ binding energies in nuclear matter. In Table XIII, we show the potential energies $U_{\Lambda}\left(\rho_{0}\right)$ for a zeromomentum $\Lambda$ and their partial-wave contributions in ${ }^{2 S+1} L_{J}$ states at normal density $\rho_{0}\left(k_{F}=1.35 \mathrm{fm}^{-1}\right)$ in the CON choice, where a statistical factor $(2 J+1)$ is included in each contribution in ${ }^{2 S+1} L_{J}$ state. The value of $U_{\Lambda}$ for $\mathrm{ESC} 16^{+}$is rather less attractive than that for ESC16, because repulsive contributions of MPP are canceled partially by attractive TBA contribution. Here, the value of $V_{\mathrm{TBA}}^{0}$ is chosen so as to reproduce $B_{\Lambda}$ values of observed $\Lambda$ hypernuclei, as shown in next subsection. The contributions to $U_{\Lambda}$ from $S$ state spinspin components can be seen qualitatively in values of $U_{\sigma \sigma}=$ $\left[U_{\Lambda}\left({ }^{3} S_{1}\right)-3 U_{\Lambda}\left({ }^{1} S_{0}\right)\right] / 12$. These values of $U_{\sigma \sigma}$ also are given in Table XIII. In the same treatment, we obtain $U_{\sigma \sigma}=1.54$ and $0.92 \mathrm{MeV}$ for NSC97f and NSC97e, respectively. Various analyses suggest that the reasonable value of $U_{\sigma \sigma}$ is between these values [57]. The $U_{\sigma \sigma}$ values for $\mathrm{ESC} 16 / \mathrm{c}^{+}$seem to be slightly too small compared to this value.

For applications to various hypernuclear problems, it is convenient to construct $k_{F}$-dependent effective local potentials $\mathcal{G}\left(k_{F} ; r\right)$ simulating the $G$ matrices in coordinate space, called YNG. Here we parametrize them in a three-range Gaussian form:

$$
\mathcal{G}\left(k_{F}, r\right)=\sum_{i=1}^{3}\left(a_{i}+b_{i} k_{F}+c_{i} k_{F}^{2}\right) \exp \left(-r^{2} / \beta_{i}^{2}\right) .
$$

The parameters $\left(a_{i}, b_{i}, c_{i}\right)$ are determined so as to simulate the calculated $G$ matrix for each ${ }^{2 S+1} L_{J}$ state. The procedures to fit the parameters are given in Ref. [57]. The obtained parameters for ESC16 are shown in Table XIV. For $\mathrm{ESC}^{+} 6^{+}$, contributions from MPP+TBA are represented by modifying the second-range parts of $\mathcal{G}\left(k_{F}, r\right)$ for ESC16 by $\Delta \mathcal{G}\left(k_{F}, r\right)=\left(a+b k_{F}+c k_{F}^{2}\right) \exp -(r / 0.9)^{2}$. The parameters for $\Delta \mathcal{G}\left(k_{F}, r\right)$ are given in Table XV. Then, the $G$-matrix interaction for $\mathrm{ESC}^{+} 6^{+}$is given by $\mathcal{G}\left(k_{F}, r\right)+\Delta \mathcal{G}\left(k_{F}, r\right)$.

Here, it is worthwhile to comment about a qualitative feature of $\Delta \mathcal{G}\left(k_{F}, r\right)$. The MPP contributions increase rapidly with matter density: In high- (low-) density region, they are very large (small), and they are cancelled considerably by TBA in the normal-density region. Then, net contributions of MPP+TBA given by $\Delta \mathcal{G}\left(k_{F}, r\right)$ are attractive for smaller values of $k_{F}$ than $1.35 \mathrm{fm}^{-1}$.

The solved $G$ matrices include not only $\Lambda N-\Lambda N$ diagonal parts but also $\Lambda N-\Sigma N$ coupling parts, and it is possible to extract such coupling parts to treat $\Lambda N-\Sigma N$ mixing problems. 

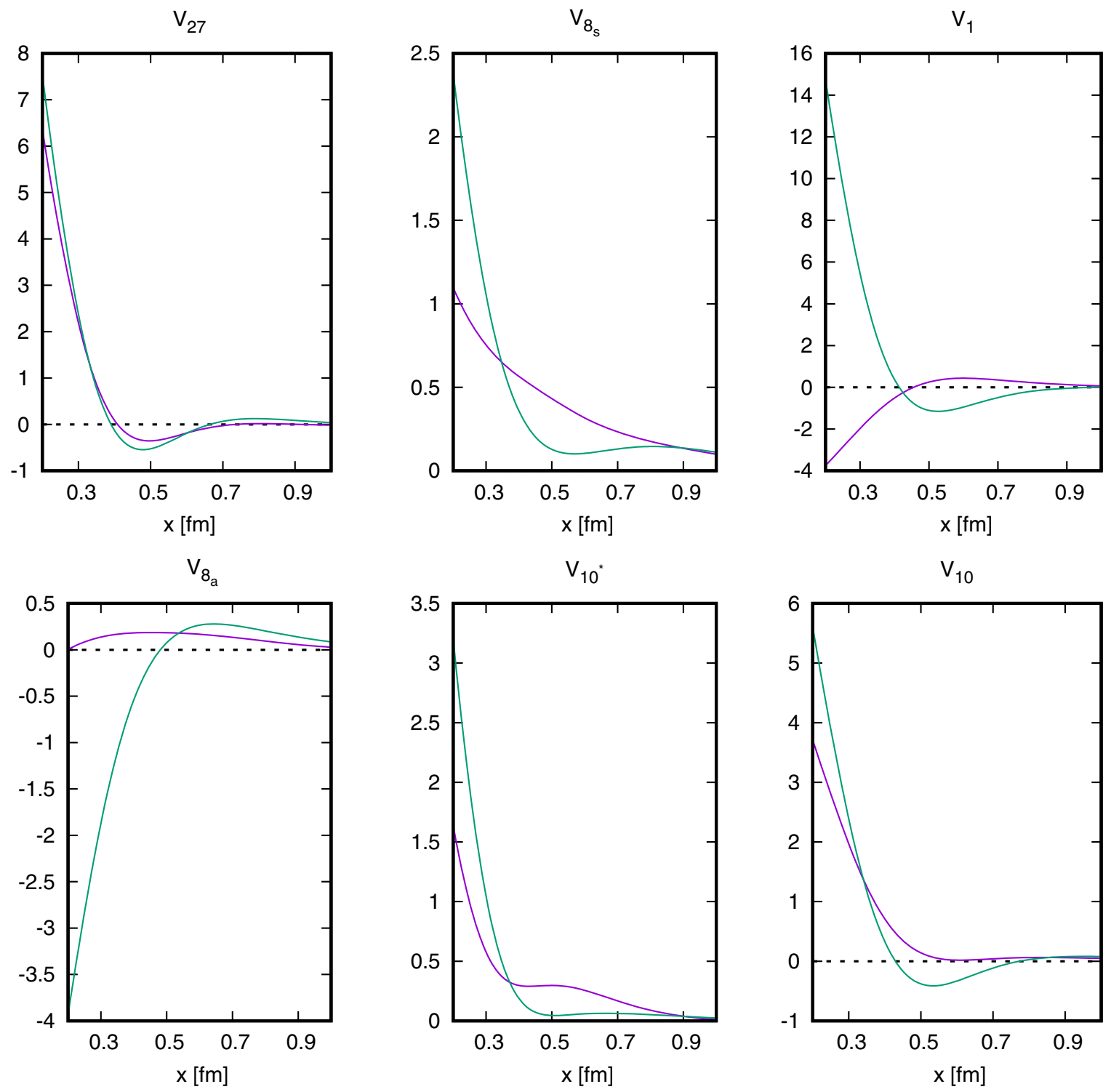

FIG. 5. Potentials in the SU(3) irreps. The solid and dashed curves are potentials with and without SU(3) symmetry breaking respectively. The units of the vertical axes are in $\mathrm{GeV}$.

The $\Lambda N-\Sigma N$ coupling interaction is determined so that its matrix elements in $k$ space simulate the corresponding $G$ matrix elements and its radial form tend to that of the bare interaction in the outermost region. In Table XVI (Table XVII), the parameters of the central (tensor) parts of $\Lambda N-\Sigma N$ and

TABLE XIII. Values of $U_{\Lambda}\left(\rho_{0}\right)$ and partial wave contributions in ${ }^{2 S+1} L_{J}$ states from the $G$-matrix calculations (in $\mathrm{MeV}$ ). The value specified by $D$ gives the sum of ${ }^{2 S+1} D_{J}$ contributions. Contributions from $S$-state spin-spin interactions are given by $U_{\sigma \sigma}=\left[U_{\Lambda}\left({ }^{3} S_{1}\right)-\right.$ $\left.3 U_{\Lambda}\left({ }^{1} S_{0}\right)\right] / 12$.

\begin{tabular}{lccccccccc}
\hline \hline & ${ }^{1} S_{0}$ & ${ }^{3} S_{1}$ & ${ }^{1} P_{1}$ & ${ }^{3} P_{0}$ & ${ }^{3} P_{1}$ & ${ }^{3} P_{2}$ & $D$ & $U_{\Lambda}$ & $U_{\sigma \sigma}$ \\
\hline ESC16 & -13.3 & -30.0 & 2.3 & 0.1 & 1.1 & -2.3 & -1.6 & -43.7 & 0.83 \\
ESC16 $^{+}$ & -12.3 & -27.4 & 2.9 & 0.3 & 1.7 & -1.2 & -1.9 & -37.9 & 0.79 \\
\hline \hline
\end{tabular}

TABLE XIV. Parameters of YNG-ESC16 continuous choice: $\mathcal{G}\left(k_{F} ; r\right)=\sum_{i=1}^{3}\left(a_{i}+b_{i} k_{F}+c_{i} k_{F}^{2}\right) \exp -\left(r / \beta_{i}\right)^{2}$.

\begin{tabular}{ccccc}
\hline \hline & $\beta_{i}$ & 0.50 & 0.90 & 2.00 \\
\hline \multirow{3}{*}{${ }^{1} E$} & $\mathrm{a}$ & -3548 & 413.2 & -1.787 \\
& $\mathrm{~b}$ & 7135 & -1087 & 0.0 \\
& $\mathrm{c}$ & -2723 & 428.5 & 0.0 \\
${ }^{3} E$ & $\mathrm{a}$ & -2820 & 325.1 & -1.372 \\
& $\mathrm{~b}$ & 5888 & -909.3 & 0.0 \\
& $\mathrm{c}$ & -2434 & 397.6 & 0.0 \\
${ }^{1} O$ & $\mathrm{a}$ & 1635 & 88.84 & -.9019 \\
& $\mathrm{~b}$ & -338.7 & 45.01 & 0.0 \\
& $\mathrm{c}$ & 138.2 & -2.532 & 0.0 \\
${ }^{3} O$ & $\mathrm{a}$ & 2283 & -223.6 & -1.070 \\
& $\mathrm{~b}$ & -2359 & 204.7 & 0.0 \\
& $\mathrm{c}$ & 823.6 & -45.50 & 0.0 \\
\hline \hline
\end{tabular}


TABLE XV. Parameters of the additional interaction in YNG$\mathrm{ESC}_{16}{ }^{+}: \Delta \mathcal{G}\left(k_{F} ; r\right)=\left(a+b k_{F}+c k_{F}^{2}\right) \exp -(r / 0.9)^{2}$.

\begin{tabular}{lrrrr}
\hline \hline & \multicolumn{1}{c}{${ }^{1} E$} & \multicolumn{1}{c}{${ }^{3} E$} & \multicolumn{1}{c}{${ }^{1} O$} & \multicolumn{1}{c}{${ }^{3} O$} \\
\hline $\mathrm{a}$ & 18.23 & 16.54 & 27.78 & 26.20 \\
$\mathrm{~b}$ & -45.62 & -42.85 & -78.02 & -75.33 \\
$\mathrm{c}$ & 27.46 & 25.80 & 69.16 & 70.44 \\
\hline \hline
\end{tabular}

$\Sigma N-\Sigma N$ interactions in $S$ states are given in a three-range Gaussian ( $r^{2}$-Gaussian) form. Here, the $k_{F}$ dependences are represented in the same form as the above diagonal parts. These coupling interactions can be used for $\Lambda N-\Sigma N$ mixing problems together with the $\Lambda N-\Lambda N$ diagonal interactions in Table XIV.

In terms of the $G$ matrices $\mathcal{G}_{L L^{\prime}}^{J S}(r)$ with $S=1$, the symmetric spin-orbit (SLS) interactions are given by the linear combination $\mathcal{G}_{\mathrm{SLS}}(r)=\left(-2 \mathcal{G}_{11}^{01}-3 \mathcal{G}_{11}^{11}+5 \mathcal{G}_{11}^{21}\right)(r) / 12$. The antisymmetric spin-orbit (ALS) $G$-matrix interaction $\mathcal{G}_{\text {ALS }}$ between ${ }^{3} P_{1}$ and ${ }^{1} P_{1}$ states is given so that its matrix elements in $k$ space simulate the corresponding $G$-matrix elements $\left\langle{ }^{3} P_{1}\right|$ $G\left|{ }^{1} P_{1}\right\rangle$. Because $\left\langle{ }^{3} P_{1}|G|{ }^{1} P_{1}\right\rangle$ and $\left\langle{ }^{1} P_{1}|G|{ }^{3} P_{1}\right\rangle$ are different from each other, we derive $\mathcal{G}_{\mathrm{ALS}}$ from their averaged values. The SLS and ALS $G$-matrix interactions obtained as a function of $k_{F}$ are represented in three-range Gaussian forms, the parameters of which are given for ESC16 in Table XVIII.

In order to compare clearly the SLS and ALS components, it is convenient to derive the strengths of the $\Lambda l-s$ potentials in hypernuclei. In the same way as in Refs. [7,14], the expression can be derived with the Scheerbaum approximation [61] as $U_{\Lambda}^{l s}(r)=K_{\Lambda} \frac{1}{r} \frac{d \rho}{d r} \mathbf{L} \cdot \mathbf{S}$. The values of $K_{\Lambda}$ can be calculated with use of $\mathcal{G}_{\mathrm{SLS}}(r)$ and $\mathcal{G}_{\mathrm{ALS}}(r)$ : The obtained value at $k_{F}=$ $1.0 \mathrm{fm}^{-1}$ is $3.8 \mathrm{MeV}, \mathrm{fm}^{5}$. This value is smaller than those for not only NSC97e/f but also ESC08a/b [57].

\section{B. $\Lambda$ hypernuclei by $\boldsymbol{G}$-matrix folding potentials}

The YNG $\Lambda N G$-matrix interaction given by Table XIV is expressed as $\mathcal{G}_{( \pm)}^{S}(r)$, with $S$ and $( \pm)$ denoting spin and

TABLE XVI. Central coupling parts of $G$-matrix interactions for ESC16, represented in a Gaussian form $\sum_{i=1}^{3}\left(a_{i}+b_{i} k_{F}+\right.$ $\left.c_{i} k_{F}^{2}\right) \exp \left[-\left(r / \beta_{i}\right)^{2}\right]$.

\begin{tabular}{lcccc}
\hline \hline & $\beta_{i}$ & 0.50 & 0.90 & 2.00 \\
\hline \multirow{2}{*}{$\Lambda N-\Sigma N{ }^{1} S_{0}$} & $\mathrm{a}$ & 5254 & -796.7 & 8.509 \\
& $\mathrm{~b}$ & -8049 & 1302 & 0.0 \\
& $\mathrm{c}$ & 3126 & -497.9 & 0.0 \\
$\Sigma N-\Sigma N{ }^{1} S_{0}$ & $\mathrm{a}$ & -365.9 & 167.5 & 8.606 \\
& $\mathrm{~b}$ & 881.7 & -72.20 & 0.0 \\
& $\mathrm{c}$ & -354.3 & 60.39 & 0.0 \\
$\Lambda N-\Sigma N{ }^{3} S_{1}$ & $\mathrm{a}$ & -2868 & 393.9 & -2.740 \\
& $\mathrm{~b}$ & 4683 & -729.1 & 0.0 \\
& $\mathrm{c}$ & -1978 & 320.5 & 0.0 \\
$\Sigma N-\Sigma N{ }^{3} S_{1}$ & $\mathrm{a}$ & 773.5 & -156.2 & -4.313 \\
& $\mathrm{~b}$ & 159.0 & -10.37 & 0.0 \\
& $\mathrm{c}$ & -172.2 & 31.04 & 0.0 \\
\hline \hline
\end{tabular}

TABLE XVII. Tensor coupling parts of $G$-matrix interactions for ESC16, represented in a $r^{2}$-Gaussian form $\sum_{i=1}^{3}\left(a_{i}+b_{i} k_{F}+\right.$ $\left.c_{i} k_{F}^{2}\right) r^{2} \exp \left[-\left(r / \beta_{i}\right)^{2}\right]$.

\begin{tabular}{ccccc}
\hline \hline & $\beta_{i}$ & 0.50 & 0.90 & 2.00 \\
\hline \multirow{4}{*}{$\Lambda N-\Sigma N{ }^{3} S_{1}$} & $\mathrm{a}$ & -44610 & 547.5 & -.7435 \\
& $\mathrm{~b}$ & 69890 & -1018 & 0.0 \\
& $\mathrm{c}$ & -26870 & 389.0 & 0.0 \\
$\Lambda N-\Lambda N{ }^{3} S_{1}$ & $\mathrm{a}$ & -2476 & 25.46 & -.0220 \\
& $\mathrm{~b}$ & 3179 & -28.42 & 0.0 \\
& $\mathrm{c}$ & -677.6 & -.5974 & 0.0 \\
\hline \hline
\end{tabular}

party quantum numbers, respectively. A $\Lambda$-nucleus potential in a finite system is derived from this $\Lambda N$ interaction by the expression

$$
\begin{aligned}
U_{\Lambda}\left(\mathbf{r}, \mathbf{r}^{\prime}\right) & =U_{d r}+U_{e x} \\
U_{d r} & =\delta\left(\mathbf{r}-\mathbf{r}^{\prime}\right) \int d \mathbf{r}^{\prime \prime} \rho\left(\mathbf{r}^{\prime \prime}\right) V_{d r}\left(\left|\mathbf{r}-\mathbf{r}^{\prime \prime}\right| ; k_{F}\right) \\
U_{e x} & =\rho\left(\mathbf{r}, \mathbf{r}^{\prime}\right) V_{e x}\left(\left|\mathbf{r}-\mathbf{r}^{\prime}\right| ; k_{F}\right) \\
\left(\begin{array}{l}
V_{d r} \\
V_{e x}
\end{array}\right) & =\frac{1}{4} \sum_{S=0,1}(2 S+1)\left[\mathcal{G}_{( \pm)}^{S} \pm \mathcal{G}_{(\mp)}^{S}\right]
\end{aligned}
$$

Here, densities $\rho(r)$ and mixed densities $\rho\left(r, r^{\prime}\right)$ are obtained from spherical Skyrme-HF wave functions.

An important problem is how to treat $k_{F}$ values included in $G$-matrix interactions. We use here the following averageddensity approximation (ADA), where an averaged value $\langle\rho\rangle$ is calculated by $\left\langle\phi_{\Lambda}(r)|\rho(r)| \phi_{\Lambda}(r)\right\rangle$ for each $\Lambda$ state $\phi_{\Lambda}(r)$, and $\left\langle k_{F}\right\rangle$ is obtained by $\left(1.5 \pi^{2}\langle\rho\rangle\right)^{1 / 3}$.

Let us calculate the energy spectra of $\Lambda$ hypernuclei systematically $\left({ }_{\Lambda}^{13} \mathrm{C},{ }_{\Lambda}^{16} \mathrm{O},{ }_{\Lambda}^{28} \mathrm{Si},{ }_{\Lambda}^{51} \mathrm{~V},{ }_{\Lambda}^{89} \mathrm{Y},{ }_{\Lambda}^{139} \mathrm{La},{ }_{\Lambda}^{208} \mathrm{~Pb}\right)$. In Fig. 6, the calculated values are compared with the experimental values marked by open circles, with the horizontal axis being given as $A^{-2 / 3}$, where solid and dashed curves are for YNG$\mathrm{ESC}_{16}{ }^{+}$and NG-ESC16, respectively. Here, the experimental data are shifted by $0.5 \mathrm{MeV}$ from the values given in Ref. [16], which has been recently proposed according to the improved calibration [62]. The $G$-matrix interaction for ESC16 is found to be overbound experimental values of $B_{\Lambda}$. In $\mathrm{ESC} 16^{+}$values of $V_{\mathrm{TBA}}^{0}=-16.0 \mathrm{MeV}$ with $\eta=4.0 \mathrm{fm}^{3}$ are chosen so that the value of $B_{\Lambda}\left({ }_{\Lambda}^{89} Y\right)$ is reproduced well and the global fitting

TABLE XVIII. Parameters of SLS and ALS $G$-matrix interactions represented by three-range Gaussian forms $\mathcal{G}\left(r ; k_{F}\right)=\sum_{i}\left(a_{i}+\right.$ $\left.b_{i} k_{F}+c_{i} k_{F}^{2}\right) \exp \left[-\left(r / \beta_{i}\right)^{2}\right]$ in the cases of ESC16.

\begin{tabular}{ccccc}
\hline \hline & $\beta_{i}$ & 0.40 & 0.80 & 1.20 \\
\hline \multirow{3}{*}{ SLS } & $\mathrm{a}$ & -11820 & 355.7 & -1.541 \\
& $\mathrm{~b}$ & 23600 & -810.3 & 0.0 \\
& $\mathrm{c}$ & -9796 & 325.2 & 0.0 \\
\multirow{3}{*}{ ALS } & $\mathrm{a}$ & 1809 & 1.423 & .7805 \\
& $\mathrm{~b}$ & -1547 & 37.07 & 0.0 \\
& $\mathrm{c}$ & 578.0 & -15.73 & 0.0 \\
\hline \hline
\end{tabular}




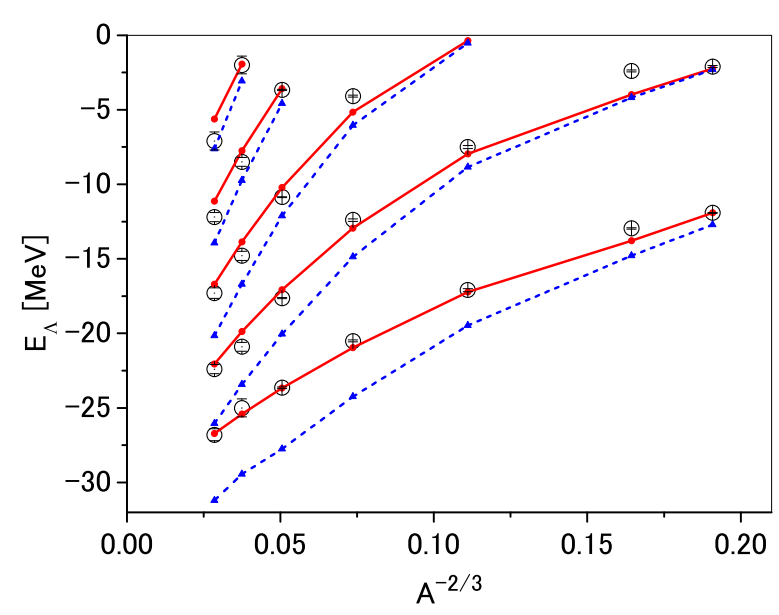

FIG. 6. Energy spectra of ${ }_{\Lambda}^{13} \mathrm{C},{ }_{\Lambda}^{28} \mathrm{Si},{ }_{\Lambda}^{51} \mathrm{~V},{ }_{\Lambda}^{89} \mathrm{Y},{ }_{\Lambda}^{139} \mathrm{La}$, and ${ }_{\Lambda}^{208} \mathrm{~Pb}$ are given as a function of $A^{-2 / 3}$, where $A$ is mass numbers of core nuclei. Solid (dashed) lines show calculated values by the $G$-matrix folding model derived from ESC $16^{+}$(ESC16). Open circles and error bars denote the experimental values taken from Ref. [16].

of $B_{\Lambda}$ values is nicely improved in comparison with that for ESC16.

The difference between $\mathrm{ESC} 16^{+}$and $\mathrm{ESC} 16$ is due to the extra terms $\Delta \mathcal{G}\left(k_{F}, r\right)$ originated from MPP+TBA. In particular, MPP plays an essential role to reproduce the nuclear saturation property and the stiffness of the EoS of neutron-star matter [58-60]. Then, it is very important that $\mathrm{ESC} 16^{+}$gives better fitting than ESC16: The density-dependent attraction $\Delta \mathcal{G}\left(k_{F}, r\right)$ in a low-density region works to reproduce better the energy spectra of heavy systems and $B_{\Lambda}$ values of light systems. In the high-density region, this extra term is dominated by MPP and leads to the stiff EoS of the hyperon-mixed neutron-star matter $[59,60]$. The present result suggests that such an effect of MPP+TBA is based on terrestrial data of $B_{\Lambda}$ values.

Finally, we comment that the $\Lambda$ s.p. energies in finite systems are not related simply to the $U_{\Lambda}\left(\rho_{0}\right)$ values given in Table XIII. The $U_{\Lambda}\left(\rho_{0}\right)$ values of $-43.7 \mathrm{MeV}(-37.9 \mathrm{MeV})$ for $\mathrm{ESC} 16\left(\mathrm{ESC} 16^{+}\right)$are very attractive compared to the value of $-30 \mathrm{MeV}$, which is the depth $U_{\mathrm{WS}}$ of the $\Lambda$ WoodsSaxon (WS) potential suitable to the data of $\Lambda$ hypernuclei [63]. However, it is misleading to compare the $U_{\Lambda}\left(\rho_{0}\right)$ value directly to the $U_{\mathrm{WS}}$ one. The $\Lambda$-nucleus folding potential depends not only on the strengths of $\Lambda N G$ matrices but also on their $k_{F}$ dependences. Then, it is only of qualitative meaning to consider the depth $U_{\mathrm{Ws}}$ of the phenomenological Woods-Saxon potential of $\Lambda$ as the $\Lambda$ potential depth in nuclear matter.

\section{C. $\Sigma N G$ matrix}

Here, we study here $\Sigma$ binding energies in nuclear matter by solving the $\Sigma N$ starting channel $G$-matrix equation. The universal repulsion MPP has to work also in $\Sigma N$ channels. Then, the problem is how to choose the phenomenological TBA part on the basis of experimental information. The positive values of $U_{\Sigma}\left(\rho_{0}\right)$ can be compared roughly with the repulsive component of the $\Sigma$ nuclear potential obtained from analyzing strong-interaction shifts and widths in $\Sigma^{-}$atoms [64]. The size of repulsion is model dependent, giving rise to the estimation of $30 \pm 20 \mathrm{MeV}$. Another experimental information for the repulsive $\Sigma$-nucleus potentials are given by the observed $\left(\pi^{-}, K^{-}\right)$spectra $[23,65,66]$. In Ref. [65], they performed the DWIA analysis for the data of ${ }^{28} \mathrm{Si}\left(\pi^{-}, K^{-}\right)$ reaction, where some $\Sigma N$ interaction models were studied. The experimental spectrum was reproduced nicely by the $\Sigma$ nucleus potential obtained from $G$ matrices for the Nijmegen model $\mathrm{F}$ [67] with the local density approximation, where the value of $U_{\Sigma}\left(\rho_{0}\right)$ was $24 \mathrm{MeV}$. Considering that the experimentally suggested values of $U_{\Sigma}\left(\rho_{0}\right)$ are strongly repulsive, we take $V_{\mathrm{TBA}}^{0}=0.0 \mathrm{MeV}$ : ESC16 ${ }^{+}$in $\Sigma N$ channels is given by ESC16+MPP without TBA.

In Table XIX, we show the potential energies $U_{\Sigma}\left(\rho_{0}\right)$ for a zero-momentum $\Sigma$ and their partial-wave contributions in $\left({ }^{2 S+1} L_{J}, T\right)$ states for ESC16 and ESC16 ${ }^{+}$. It should be noted here that the strongly repulsive contributions in ${ }^{3} S_{1} T=$ $3 / 2$ and ${ }^{1} S_{0} T=1 / 2$ states are due to the Pauli-forbidden effects in these states, taken into account by strengthening the Pomeron coupling in the ESC16 modeling.

In the left (right) panel of Fig. 7, $U_{\Sigma}$ values (their $S$-state contributions) are drawn as a function of $k_{F}$ for ESC16 and $\mathrm{ESC} 6^{+}$by dashed and solid curves, respectively. It is demonstrated that the repulsive $U_{\Sigma}$ values are due to $T=3 / 2{ }^{3} S_{1}$ and $T=1 / 2{ }^{1} S_{0}$ contributions, and the repulsions are enhanced by the MPP contributions.

The value of $U_{\Sigma}$ is sensitive to the Pauli-repulsion parameter $a_{\mathrm{PB}}$. Though $a_{\mathrm{PB}}=0.39$ is taken in ESC16, a larger value of $a_{\mathrm{PB}}$ gives rise to a more repulsive value of $U_{\Sigma}$. Taking $a_{\mathrm{PB}}=0.59$, we obtain $U_{\Sigma}\left(\rho_{0}\right)=7.4 \mathrm{MeV}$ and $20.3 \mathrm{MeV}$ for ESC16 and $\mathrm{ESC}_{16}{ }^{+}$, respectively. As found in Table VII, such a high value for the Pauli-blocking repulsion gives $\Sigma^{+} p$ cross sections that are too high. In order to obtain strongly repulsive values of $U_{\Sigma}$ without overestimating $\Sigma^{+} p$ cross sections, it might be necessary to introduce further many-body repulsions.

\section{DISCUSSION, CONCLUSIONS, AND OUTLOOK}

We have again shown in this paper that the ESC approach to the nuclear force problem is able to make a connection

TABLE XIX. Values of $U_{\Sigma}\left(\rho_{0}\right)$ at normal density and partial wave contributions in $\left({ }^{2 S+1} L_{J}, T\right)$ states for ESC16/c $($ in MeV).

\begin{tabular}{lccccccccc}
\hline \hline Model & $T$ & ${ }^{1} S_{0}$ & ${ }^{3} S_{1}$ & ${ }^{1} P_{1}$ & ${ }^{3} P_{0}$ & ${ }^{3} P_{1}$ & ${ }^{3} P_{2}$ & $U_{\Sigma}$ \\
\hline \multirow{2}{*}{ ESC16 } & $1 / 2$ & 10.2 & -24.7 & 1.9 & 2.1 & -5.3 & -0.2 & -0.6 \\
& $3 / 2$ & -13.1 & 29.5 & -3.5 & -2.1 & 5.3 & -2.6 & -0.2 \\
\multirow{2}{*}{ ESC16 $^{+}$} & $1 / 2$ & 10.8 & -20.7 & 2.1 & 2.2 & -5.0 & 0.2 & -0.5 & -3.3 \\
& $3 / 2$ & -11.4 & 33.3 & -3.0 & -2.0 & 5.6 & -1.8 & -0.0 & +9.9 \\
\hline \hline
\end{tabular}



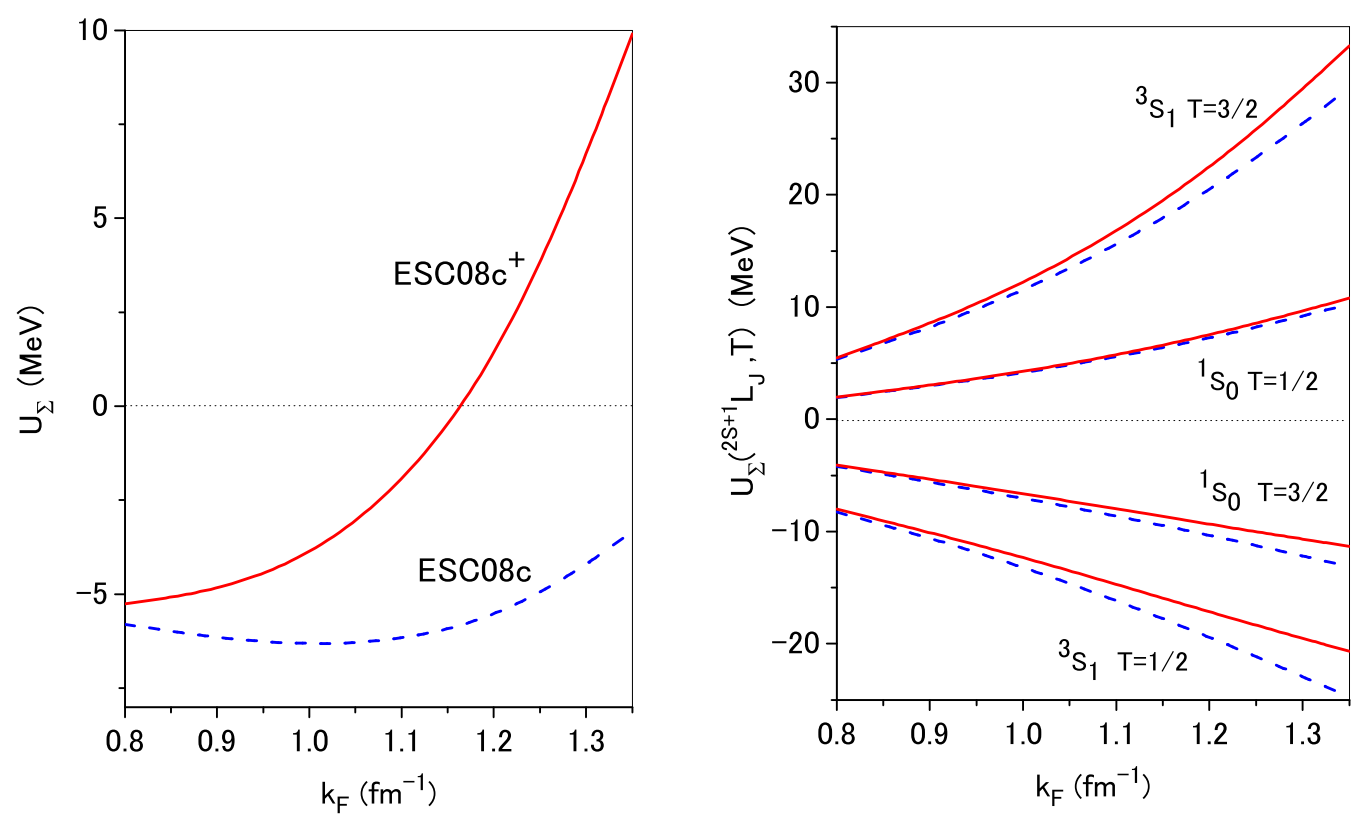

FIG. 7. In the left (right) panel, the values of $U_{\Sigma}$ (partial-wave contributions) are drawn as a function of $k_{F}$ by dashed and solid curves for $\mathrm{ESC} 16$ and $\mathrm{ESC} 16^{+}$, respectively.

between on the one hand the presently available baryonbaryon data and on the other hand the underlying quark structure of the baryons and mesons. Namely, a very successful description of both the $N N$ - and $Y N$-scattering data is obtained with meson-baryon coupling parameters which are almost all explained by the QPC model, and at the same time in obedience to the strong constraint of no bound states in the $S=-1$ systems. Therefore, the ESC16 model of this paper is an important further step in the determination of the baryon-baryon interactions for low-energy scattering and the description of hypernuclei in the context of broken SU(3) symmetry. The values for many parameters, which in previous work were considered to be free to a large extent, are now limited strongly and made consistent with the present theoretical view on low-energy hadron physics. This is in particular the case for the $F /(F+D)$ ratios of the MPE interactions. These ratios for the vector and scalar mesons are rather close to the QPC-model predictions.

In analyzing the effect of the Pauli-blocking repulsion, the conclusion is that from the standpoint of the $B B$ scattering data fitting such a repulsion is not strong. This conclusion is in line with arguments from strong-coupling QCD (SCQCD). Namely, it has been argued in Ref. [68] that quark-exchange effects are small.

The $G$-matrix results show that basic features of hypernuclear data are reproduced nicely by ESC16, improving the weak points of the soft-core OBE models NSC89 [13], NSC97 [14], and ESC04- [6-8]. The ESC16 model is superior for hypernuclear data and many aspects of the effective (two-body) interactions in hypernuclei can be obtained using the ESC16 model. For example, this is the case for the well depth $U_{\Sigma}$.

Experience has shown that a good fit to the scattering data not necessarily means success in the $G$-matrix results. To explain this, one can think of two reasons: (i) the $G$-matrix results are sensitive to the two-body interactions below 1 $\mathrm{fm}$, whereas the present $Y N$-scattering data are not, and (ii) other than two-body forces play an important role. However, since the $N N \oplus Y N$ fit is so much superior for ESC16 than for OBE models, we are inclined to look for solutions to the remaining problems outside the two-body forces. A natural possibility is the presence of three-body forces (TBF) in hypernuclei which can be viewed as generating effective twobody forces, which could solve the well-depth issues. In the case of the $\Delta B_{\Lambda \Lambda}$ also TBF could be operating. This calls for an evaluation of the TBF's $N N N, \Lambda N N, \Sigma N N, \Lambda \Lambda N$, etc., for the soft-core ESC model, consistent with its two-body forces.

The $\Lambda N p$ waves seem to be better, which is the result of the truly simultaneous $N N \oplus Y N$ fitting. This is also reflected in the better $K_{\Lambda}$ value, making the well-known small spinorbit splitting smaller.

In the course of the development of the ESC model for baryon-baryon, up to and including ESC06 [69], solving all problems for $N N$ and $Y N$ was tried, both for scattering and hypernuclear well depths, by keeping the potentials restricted to meson exchange. For that purpose, in ESC06 a superextended ESC approach was studied by including the second generation of the mesons, i.e., the heavy pseudoscalar, vector, and scalar meson nonets. In the quark model, they would correspond to the first radially excited $q \bar{q}$ states, with masses in the range $1 \mathrm{GeV} / \mathrm{c}^{2}<m<1.7 \mathrm{GeV} / \mathrm{c}^{2}$. With this extension, it is possible to produce extra repulsion in the $\Sigma^{+} p\left({ }^{3} S_{1}\right)$, but correlated with this was an extremely strong attraction in the $\Sigma^{+} p\left({ }^{1} P_{1}\right)$ partial wave. Although the ESC06 approach is not ruled out by the data, we think that the solutions presented with ESC16 are much superior. In the future, such a superextended ESC16 model may be explored. For example, 
the axial-vector and heavy pseudoscalar $[\pi(1300)]$ meson sectors can be studied more closely.

\section{ACKNOWLEDGMENT}

We wish to thank E. Hiyama, K. Itonaga, T. Motoba, and H.-J. Schulze for many stimulating discussions.

\section{APPENDIX A: TREATMENT WIDTHS SCALAR AND VECTOR MESONS}

The effects of the large width of the vector $\rho(760)$ meson and the scalar $\epsilon(620)$ and $\kappa(861)$ mesons are taken into account via a generalization of the narrow (i.e., stable) meson propagator through the Källen-Lehman representation. For a narrow meson, the propagator is $\left(k_{0}=0\right)$

$$
\Delta\left(\mathbf{k}^{2}\right)=\frac{1}{\mathbf{k}^{2}+m^{2}-i \delta}=\int_{m_{0}^{2}}^{\infty} d m^{\prime 2} \frac{\rho\left(m^{\prime 2}\right)}{\mathbf{k}^{2}+m^{\prime 2}-i \delta},
$$

with the spectral function $\rho\left(m^{\prime 2}\right)=\delta\left(m^{\prime 2}-m^{2}\right)$. Here, $m_{0}^{2}$ denotes the branch point of $\Delta\left(k^{2}\right)$ in the complex $k^{2}$ plane. For the broad mesons, we use the spectral functions [70,71]

$$
\rho\left(m^{\prime 2}\right)=\frac{1}{\pi} \frac{\gamma\left(m^{\prime 2}-m_{0}^{2}\right)^{n+1 / 2} \theta\left(m^{\prime 2}-m_{0}^{2}\right)}{\left(m^{\prime 2}-m^{2}\right)^{2}+\gamma^{2}\left(\frac{m^{\prime 2}}{m^{2}}\right)^{2 n}\left(m^{\prime 2}-m_{0}^{2}\right)^{2 n+1}},
$$

with $n=1$ for the $\rho$ and $n=0$ for $\epsilon$ and $\kappa$. Furthermore, $m_{0}^{2}=4 m_{\pi}^{2}$ and $m_{0}^{2}=\left(m_{\pi}+m_{K}\right)^{2}$ for respectively the $\rho, \epsilon$ and $\kappa(861)$. The $\gamma$ entity contains the meson width $\Gamma$ and is defined as

$$
\gamma=m \Gamma /\left(m^{2}-m_{0}^{2}\right)^{n+1 / 2} .
$$

Substituting (A2) into (A1) gives for $n=0$

$$
\Delta\left(\mathbf{k}^{2}\right)=\left[\mathbf{k}^{2}+m^{2}+\gamma\left(\frac{\mathbf{k}^{2}}{m^{2}}\right)^{n}\left(\mathbf{k}^{2}+m_{0}^{2}\right)^{n+1 / 2}\right]^{-1} .
$$

This formula is approximately also correct for $n=1$ for not too large $\mathbf{k}^{2}$ (see Refs. [71,72]). Note the following properties of (A4):

(i) It has a cut in the complex $\mathbf{k}^{2}$ plane with a branch point at the $(\pi \pi)$ or $(\pi K)$ threshold, connected to the decay of these mesons. Moreover, it has the proper theshold behavior at these thresholds.

(ii) It shows the correct Breit-Wigner form in the neighborhood of $\mathbf{k}^{2}=m^{2}$, with a width $\Gamma$.

After Fourier transformation to configuration space of (A1) with the spectral function $\rho\left(\mathrm{m}^{\prime 2}\right.$, one obtains for these broad mesons a (contineous) superposition of Yukawa potentials wit the mass distribution $2 m^{\prime} \rho\left(m^{\prime 2}\right)$. For the purpose of practical calculations, these potentials are approximated by the sum of two Yukawa potentials from two effective narrow mesons ("dipole" fit):

$$
\int_{m_{0}^{2}}^{\infty} d m^{\prime 2} \rho\left(m^{\prime 2}\right) \frac{e^{-m^{\prime} r}}{r} \approx \beta_{1} \frac{e^{-m_{1} r}}{r}+\beta_{2} \frac{e^{-m_{2} r}}{r} .
$$

TABLE XX. Values for the dipole approximation for the broad $\rho(760, \Gamma=120), \epsilon(620, \Gamma=464)$, and $\kappa(861, \Gamma=450)$. Masses and widths are in $\mathrm{MeV}$.

\begin{tabular}{cccccr}
\hline \hline Meson & $n$ & $\beta_{1}$ & $m_{1}$ & \multicolumn{1}{c}{$\beta_{2}$} & \multicolumn{1}{c}{$m_{2}$} \\
\hline$\rho(760)$ & 1 & 0.19068 & 647.436 & 0.79649 & 898.117 \\
$\epsilon(620)$ & 0 & 0.28193 & 455.159 & 0.718071 & 1158.562 \\
$\kappa(861)$ & 0 & 0.47824 & 813.628 & 0.521761 & 1947.286 \\
\hline \hline
\end{tabular}

Here, $m_{0}=2 m_{\pi}$ and $m_{0}=\left(m_{\pi}+m_{K}\right)$ in the case of the $(\rho, \varepsilon)$ and $\kappa$ respectively.

To determine the $\left(\beta_{1,2}, m_{1,2}\right)$ parameters, a possibility is to fit the left- and right-hand sides of (A5) for a certain range of $r$ values. We follow the Bryan-Gersten analytical procedure given in Ref. [70], which runs as follows: One writes $\left(k^{2} \equiv\right.$ $\left.\mathbf{k}^{2}\right)$

$$
\Delta\left(k^{2}\right) \approx \Delta_{\text {dip }}\left(k^{2}\right)=\frac{\beta_{1}}{k^{2}+m_{1}^{2}}+\frac{\beta_{2}}{k^{2}+m_{2}^{2}}
$$

and requires that $\Delta_{\text {dip }}=\Delta$ is satisfied for these four conditions: (1) $k^{2} \rightarrow \infty$, (2) $k^{2}=0$, (3) $k^{2}=m^{2}$, and (4) the derivatives match at $k^{2}=0$, i.e., $d \Delta /\left.d k^{2}\right|_{k^{2}=0}=$ $d \Delta_{\text {dip }} /\left.d k^{2}\right|_{k^{2}=0}$. The solution of these conditions determines the dipole parameters. The results are given in Table XX.

\section{APPENDIX B: MPE INTERACTIONS AND SU(3)}

Below, $\sigma, \mathbf{a}_{0}, \mathbf{A}_{1}, \ldots$ are shorthand notation for, respectively, the baryon $\mathrm{SU}(3)$-singlet and $\mathrm{SU}(3)$-octet densities $\bar{\psi} \psi, \bar{\psi} \lambda \psi, \bar{\psi} \gamma_{5} \gamma_{\mu} \lambda \psi, \ldots$. Here, $\lambda_{i}, \quad i=0,1, \ldots, 8$ are the Gell-Mann SU(3) matrices.

For the pseudoscalar, vector, scalar, and axial-vector mesons, the SU(3) octet and singlet states appearing in the meson pairs, denoted by the subscript 8 (respectively, 1), are in terms of the physical ones defined as follows:

(i) Pseudoscalar mesons:

$$
\begin{aligned}
& \eta_{1}=\cos \theta_{P} \eta^{\prime}-\sin \theta_{P} \eta, \\
& \eta_{8}=\sin \theta_{P} \eta^{\prime}+\cos \theta_{P} \eta .
\end{aligned}
$$

Here, $\eta^{\prime}$ and $\eta$ are the physical pseudoscalar mesons $\eta(957)$ respectively $\eta(548)$.

(ii) Vector mesons:

$$
\begin{aligned}
& \phi_{1}=\cos \theta_{V} \omega-\sin \theta_{V} \phi, \\
& \phi_{8}=\sin \theta_{V} \omega+\cos \theta_{V} \phi .
\end{aligned}
$$

Here, $\phi$ and $\omega$ are the physical vector mesons $\phi(1019)$ respectively $\omega(783)$.

Then, one has the following SU(3)-invariant pairinteraction Hamiltonians: 
(1) $J^{P C}=0^{+-}: \mathrm{SU}(3)$-singlet couplings $S_{\beta}^{\alpha}=\delta_{\beta}^{\alpha} \sigma / \sqrt{3}$,

$$
\mathcal{H}_{S_{1} P P}=\frac{g_{S_{1} P P}}{\sqrt{3}}\left\{\pi \cdot \pi+2 K^{\dagger} K+\eta_{8} \eta_{8}\right\} \sigma .
$$

(2) $J^{P C}=0^{++}: \mathrm{SU}(3)$-octet symmetric couplings I, $S_{\beta}^{\alpha}=\left(S_{8}\right)_{\beta}^{\alpha} \Rightarrow(1 / 4) \operatorname{Tr}\left\{S[P, P]_{+}\right\}$,

$$
\begin{aligned}
\mathcal{H}_{S_{8} P P}= & \frac{g_{S_{8} P P}}{\sqrt{6}}\left\{\left(\mathbf{a}_{0} \cdot \boldsymbol{\pi}\right) \eta_{8}+\frac{\sqrt{3}}{2} \mathbf{a}_{0} \cdot\left(K^{\dagger} \boldsymbol{\tau} K\right)+\frac{\sqrt{3}}{2}\left\{\left(K_{0}^{\dagger} \boldsymbol{\tau} K\right) \cdot \boldsymbol{\pi}+\text { H.c. }\right\}\right. \\
& \left.-\frac{1}{2}\left\{\left(K_{0}^{\dagger} K\right) \eta_{8}+\text { H.c. }\right\}+\frac{1}{2} f_{0}\left(\boldsymbol{\pi} \cdot \boldsymbol{\pi}-K^{\dagger} K-\eta_{8} \eta_{8}\right)\right\} .
\end{aligned}
$$

(3) $J^{P C}=1^{+-}: \mathrm{SU}(3)$-octet symmetric couplings II, $S_{\beta}^{\alpha}=\left(B_{8}\right)_{\beta}^{\alpha} \Rightarrow(1 / 4) \operatorname{Tr}\left\{B^{\mu}\left[V_{\mu}, P\right]_{+}\right\}$,

$$
\begin{aligned}
\mathcal{H}_{B_{8} V P}= & \frac{g_{B_{8} V P}}{\sqrt{6}}\left\{\frac{1}{2}\left[\left(\mathbf{B}_{1}^{\mu} \cdot \boldsymbol{\rho}_{\mu}\right) \eta_{8}+\left(\mathbf{B}_{1}^{\mu} \cdot \boldsymbol{\pi}_{\mu}\right) \phi_{8}\right]+\frac{\sqrt{3}}{4}\left[\mathbf{B}_{1} \cdot\left(K^{* \dagger} \boldsymbol{\tau} K\right)+\text { H.c. }\right]\right. \\
& +\frac{\sqrt{3}}{4}\left[\left(K_{1}^{\dagger} \boldsymbol{\tau} K^{*}\right) \cdot \boldsymbol{\pi}+\left(K_{1}^{\dagger} \boldsymbol{\tau} K\right) \cdot \boldsymbol{\rho}+\text { H.c. }\right]-\frac{1}{4}\left[\left(K_{1}^{\dagger} \cdot K^{*}\right) \eta_{8}+\left(K_{1}^{\dagger} \cdot K\right) \phi_{8}+\text { H.c. }\right] \\
& \left.+\frac{1}{2} H^{0}\left[\boldsymbol{\rho} \cdot \boldsymbol{\pi}-\frac{1}{2}\left(K^{* \dagger} \cdot K+K^{\dagger} \cdot K^{*}\right)-\phi_{8} \eta_{8}\right]\right\} .
\end{aligned}
$$

(4) $J^{P C}=1^{--}: \mathrm{SU}(3)$-octet asymmetric couplings I, $A_{\beta}^{\alpha}=\left(V_{8}\right)_{\beta}^{\alpha} \Rightarrow(-i / \sqrt{2}) \operatorname{Tr}\left\{V^{\mu}\left[P, \partial_{\mu} P\right]_{-}\right\}$,

$$
\begin{aligned}
\mathcal{H}_{V_{8} P P}= & g_{A_{8} P P}\left\{\frac{1}{2} \boldsymbol{\rho}_{\mu} \cdot \boldsymbol{\pi} \times \overleftrightarrow{\partial^{\mu}} \boldsymbol{\pi}+\frac{i}{2} \boldsymbol{\rho}_{\mu} \cdot\left(K^{\dagger} \boldsymbol{\tau} \overleftrightarrow{\partial^{\mu}} K\right)+\frac{i}{2}\left(K_{\mu}^{* \dagger} \boldsymbol{\tau}\left(K \stackrel{\leftrightarrow}{\partial^{\mu}} \boldsymbol{\pi}\right)-\text { H.c. }\right)+i \frac{\sqrt{3}}{2}\left(K_{\mu}^{* \dagger}\right.\right. \\
& \left.\left.\times\left(K \cdot \overleftrightarrow{\partial^{\mu}} \eta_{8}\right)-\text { H.c. }\right)+\frac{i}{2} \sqrt{3} \phi_{\mu}\left(K^{\dagger} \stackrel{\leftrightarrow}{\partial^{\mu}} K\right)\right\} .
\end{aligned}
$$

(5) $J^{P C}=1^{++} \mathrm{SU}(3)$-octet asymmetric couplings II, $A_{\beta}^{\alpha}=\left(A_{8}\right)_{\beta}^{\alpha} \Rightarrow(-i / \sqrt{2}) \operatorname{Tr}\left\{A^{\mu}\left[P, V_{\mu}\right]_{-}\right\}$:

$$
\begin{aligned}
\mathcal{H}_{A_{8} V P}= & g_{A_{8} V P}\left\{\mathbf{A}_{1} \cdot \boldsymbol{\pi} \times \boldsymbol{\rho}+\frac{i}{2} \mathbf{A}_{1} \cdot\left[\left(K^{\dagger} \boldsymbol{\tau} K^{*}\right)-\left(K^{* \dagger} \boldsymbol{\tau} K\right)\right]-\frac{i}{2}\left(\left[\left(K^{\dagger} \boldsymbol{\tau} K_{A}\right) \cdot \boldsymbol{\rho}+\left(K_{A}^{\dagger} \boldsymbol{\tau} K^{*}\right) \cdot \boldsymbol{\pi}\right]-\text { H.c. }\right)\right. \\
& \left.-i \frac{\sqrt{3}}{2}\left(\left[\left(K^{\dagger} \cdot K_{A}\right) \phi_{8}+\left(K_{A}^{\dagger} \cdot K^{*}\right) \eta_{8}\right]-\text { H.c. }\right)+\frac{i}{2} \sqrt{3} f_{1}\left[K^{\dagger} \cdot K^{*}-K^{* \dagger} \cdot K\right]\right\} .
\end{aligned}
$$

The relation with the pair-couplings used in this paper and Ref. [1], see also Ref. [30], is $g_{S_{1} P P} / \sqrt{3}=g_{(\pi \pi)_{0}} / m_{\pi}, g_{A_{8} V P}=$ $g_{(\pi \rho)_{1}} / m_{\pi}$.

\section{APPENDIX C: $J^{P C}=1^{+-}$AXIAL-PAIR POTENTIALS}

In this Appendix, we document the $J^{P C}=1^{+-}$axial $(\pi \omega)$ 1-pair potentials, which have not been reported elsewhere yet. The involved meson pairs can be read off from the SU(2) structure of the interaction Hamiltonian (4.27).

Below, we denote the type of potentials by writing $V_{\sigma+T}^{(n)}$, where $n=0,1$ refers to the $(1 / M)$ order and the subscript $\sigma+T$ indicates that only the spin-spin and tensor contributions are given here and not the spin-orbit potentials.

\section{$N N$ potentials, $(S=0, I=1)$ exchange, and $\left(\pi \omega_{1}\right)$}

To be specific, consider $(\pi \omega)_{1}$ exchange for $N N$ and elastic $\Sigma N$ potentials. One obtains the following:

(1) The leading, i.e., $(1 / M)^{0}$, terms in momentum and configuration space are

$$
\begin{gathered}
\widetilde{V}_{\sigma+T}^{(0)}(\mathbf{q}, \mathbf{k})=+g_{(\pi \omega)_{1} ; N N} f_{N N \pi} G_{N N \omega}\left(\sigma_{1} \cdot \mathbf{k} \sigma_{2} \cdot \mathbf{k}_{1}+\sigma_{1} \cdot \mathbf{k}_{1} \boldsymbol{\sigma}_{2} \cdot \mathbf{k}\right) \frac{1}{\omega_{1}^{2} \omega_{2}^{2}} \frac{1}{m_{\pi}^{2} \mathcal{M}}, \\
V_{\sigma+T}^{(0)}(r)=-2 g_{(\pi \omega ; N N)} f_{N N \pi} G_{N N \omega}\left[F_{B, \sigma}^{(0)}(r) \sigma_{1} \cdot \sigma_{2}+F_{B, T}^{(0)}(r) S_{12}\right] \frac{1}{m_{\pi}^{2} \mathcal{M}},
\end{gathered}
$$


where

$$
F_{B, \sigma}^{(0)}(r)=\frac{1}{3}\left(\frac{2}{r} F^{\prime} G+F^{\prime} G^{\prime}+F^{\prime \prime} G\right), \quad F_{B, T}^{(0)}(r)=\frac{1}{3}\left(-\frac{1}{r} F^{\prime} G+F^{\prime} G^{\prime}+F^{\prime \prime} G\right) .
$$

Above, $\omega_{1}=\sqrt{\mathbf{k}_{1}^{2}+m_{\pi}^{2}}$ and $\omega_{2}=\sqrt{\mathbf{k}_{2}^{2}+m_{\omega}^{2}}$. For the Fourier transforms of the momentum pair-exchange potentials with Gaussian form factors, we refer to the basic papers [30]. The superscript for the functions $F_{B, \sigma, T}$ refers to the denominators $1 /\left(\omega_{1}^{2} \omega_{2}^{2}\right)$ in Eq. (C1). For these denominators, in the notation of Ref. [30], the functions $F$ and $G$ are

$$
F(r)=I_{2}\left(r, m_{\pi}, \Lambda_{\pi}\right), \quad G(r)=I_{2}\left(r, m_{\omega}, \Lambda_{\omega}\right) .
$$

Similar formulas apply to, e.g., $\Sigma N$ potentials, and also to $\left(K^{*} K\right)_{1}$ pair exchange.

(2) The nonleading, i.e., $(1 / M)$, terms, are

$$
\begin{aligned}
\widetilde{V}_{\sigma+T}^{(1)}(\mathbf{q}, \mathbf{k}) & =-g_{(\pi \omega)_{1} ; N N} f_{N N \pi} G_{N N \omega} \frac{1}{2 M_{N}}\left(\sigma_{1} \cdot \mathbf{k} \sigma_{2} \cdot \mathbf{k}_{2}+\sigma_{1} \cdot \mathbf{k}_{2} \boldsymbol{\sigma}_{2} \cdot \mathbf{k}\right) \frac{1}{\omega_{1} \omega_{2}\left(\omega_{1}+\omega_{2}\right)} \frac{1}{m_{\pi}^{2} \mathcal{M}}, \\
V_{\sigma+T}^{(1)}(r) & =+2 g_{(\pi \omega)_{1} ; N N} f_{N N \pi} G_{N N \omega} \frac{m_{\pi}}{2 M_{N}}\left[F_{B, \sigma}^{(1)}(r) \sigma_{1} \cdot \boldsymbol{\sigma}_{2}+F_{B, T}^{(1)}(r) S_{12}\right] \frac{1}{m_{\pi}^{3} \mathcal{M}}
\end{aligned}
$$

where now superscript for the functions $F_{B, \sigma, T}^{(1)}$ refers to the denominators $1 /\left[\omega_{1} \omega_{2}\left(\omega_{1}+\omega_{2}\right)\right]$ in Eq. (C4). For this denominator, the basic Fourier transform is [30]

$$
F_{B}^{(1)}(r)=\frac{2}{\pi} \int_{0}^{\infty} d \lambda F(\Lambda, r) G(\lambda, r)
$$

where the functions $F$ and $G$ are

$$
F(r)=I_{2}\left[r, m_{\pi}(\lambda), \Lambda_{\pi}\right], \quad G(r)=I_{2}\left[r, m_{\omega}(\lambda), \Lambda_{\omega}\right],
$$

with the understanding that under the $\lambda$ integral in Eq. (C5) there occur the combinations

$$
F_{B, \sigma}^{(1)}(r)=\frac{1}{3}\left(\frac{2}{r} F G^{\prime}+F^{\prime} G^{\prime}+F G^{\prime \prime}\right), \quad F_{B, T}^{(1)}(r)=\frac{1}{3}\left(-\frac{1}{r} F G^{\prime}+F^{\prime} G^{\prime}+F G^{\prime \prime}\right) .
$$

(3) The symmetric spin-orbit $(1 / M)^{2}$ terms, are

$$
\begin{aligned}
\widetilde{V}_{\mathrm{SLS}}^{(2)}(\mathbf{q}, \mathbf{k}) & =-g_{(\pi \omega)_{1} ; N N} f_{N N \pi} G_{N N \omega} \frac{1}{M_{N}^{2}} \frac{i}{2}\left(\sigma_{1}+\boldsymbol{\sigma}_{2}\right) \cdot \mathbf{q} \cdot \mathbf{k}_{2} \cdot \frac{1}{\omega_{2}^{2}} \\
V_{\mathrm{SLS}}^{(2)}(r) & =-g_{(\pi \omega)_{1} ; N N} f_{N N \pi} G_{N N \omega} \frac{1}{m_{\pi}^{2} M_{N}^{2}} I_{0}\left(m_{\pi}, r\right)\left(-\frac{1}{r} \frac{d}{d r} I_{2}\left(m_{\omega}, \Lambda_{V}, r\right)\right) \mathbf{L} \cdot \mathbf{S}
\end{aligned}
$$

where

$$
I_{0}\left(\Lambda_{P}, r\right)=\frac{1}{4 \pi} \frac{1}{2 \sqrt{\pi}}\left(\frac{\Lambda_{P}}{m_{\pi}}\right)^{3} \exp \left(-\frac{1}{4} \Lambda_{P}^{2} r^{2}\right) .
$$

We note that important contributions to the antisymmetric spin-orbit potentials are proportional to $\left(1 / M_{N}-1 / M_{Y}\right) \sim$ $1 / M^{2}$. Also, spin-orbit potentials from OBE are order $1 / M^{2}$. Therefore, we included this SLS potential in the ESC16 model.

\section{$Y N$ potentials, $(S=0, I=0)$ exchange, and $(\pi \rho)_{0}$}

The above potentials also occur in $Y N$ and $Y Y$ channels, of course. In this subsection, we give as an illustration only the $1 / M$ contribution for the spin-spin and tensor. Again, to be specific, now we consider $(\pi \rho)_{0}$ exchange for $\Lambda N$ potentials. We obtain

$$
\begin{aligned}
\widetilde{V}_{\sigma+T}^{(1)}(\mathbf{q}, \mathbf{k})= & -2 g_{\Lambda \Lambda ;(\pi \rho)_{0}} f_{N N \pi} G_{N N \rho} \frac{1}{2 M_{N}}\left[\sigma_{1} \cdot \mathbf{k} \boldsymbol{\sigma}_{2} \cdot \mathbf{k}_{2}\right] \frac{1}{\omega_{1} \omega_{2}\left(\omega_{1}+\omega_{2}\right)} \\
& -2 g_{N N ;(\pi \rho)_{0}} f_{\Lambda \Sigma \pi} G_{\Lambda \Sigma \rho} \frac{1}{M_{\Lambda}+M_{\Sigma}}\left[\sigma_{1} \cdot \mathbf{k}_{2} \boldsymbol{\sigma}_{2} \cdot \mathbf{k}\right] \frac{1}{\omega_{1} \omega_{2}\left(\omega_{1}+\omega_{2}\right)}
\end{aligned}
$$


In configuration space, we get

$$
\begin{aligned}
V_{\sigma+T}^{(1)}(r)= & +2 g_{\Lambda \Lambda ;(\pi \rho)_{0}} f_{N N \pi} G_{N N \rho} \frac{1}{2 M_{N}}\left[G_{B, \sigma}^{(1)}(r) \sigma_{1} \cdot \sigma_{2}+G_{B, T}^{(1)}(r) S_{12}\right] \\
& +2 g_{N N ;(\pi \rho)_{0}} f_{\Lambda \Sigma \pi} G_{\Lambda \Sigma \rho} \frac{1}{M_{\Lambda}+M_{\Sigma}}\left[G_{B, \sigma}^{(1)}(r) \sigma_{1} \cdot \sigma_{2}+G_{B, T}^{(1)}(r) S_{12}\right]
\end{aligned}
$$

where

$$
\begin{aligned}
& G_{B, \sigma}^{(1)}(r)=\frac{1}{3}\left(\frac{2}{r} F_{\pi} \otimes F_{\omega}^{\prime}+F_{\pi}^{\prime} \otimes F_{\omega}^{\prime}+F_{\pi} \otimes F_{\omega}^{\prime \prime}\right), \\
& G_{B, T}^{(1)}(r)=\frac{1}{3}\left(-\frac{1}{r} F_{\pi} \otimes F_{\omega}^{\prime}+F_{\pi}^{\prime} \otimes F_{\omega}^{\prime}+F \otimes_{\pi} F_{\omega}^{\prime \prime}\right) .
\end{aligned}
$$

Here, again the superscript on the $G$ functions refers to the denominator in momentum space. For the denominators in Eq. (C10), the functions $F \otimes g$ are given by [30]

$$
F_{\alpha} \otimes F_{\beta}(r)=\frac{2}{\pi} \int_{0}^{\infty} d \lambda F_{\alpha}(\lambda, r) F_{\beta}(\lambda, r)
$$

where

$$
F_{\alpha}(\lambda, r)=e^{-\lambda^{2} / \Lambda_{\alpha}^{2}} I_{2}\left(\sqrt{m_{\alpha}^{2}+\lambda^{2}}, r\right)
$$

$Y N$ potentials, $(S= \pm 1, I=1 / 2)$ exchange, and $\left(\pi K^{*}\right)_{1 / 2}$

Again, to be specific, consider $\left(\pi K^{*}\right)_{1 / 2}$ exchange for $\Lambda N$ potentials. One obtains the leading, i.e., $(1 / M)^{0}$, potentials

$$
\begin{aligned}
\widetilde{V}_{\sigma+T}^{(0)}(\mathbf{q}, \mathbf{k})= & +g_{\left(\pi K^{*}\right) ; \Lambda N} f_{N N \pi} G_{N \Lambda K^{*}}\left(\boldsymbol{\sigma}_{1} \cdot \mathbf{k} \boldsymbol{\sigma}_{2} \cdot \mathbf{k}_{1}+\boldsymbol{\sigma}_{1} \cdot \mathbf{k}_{1} \boldsymbol{\sigma}_{2} \cdot \mathbf{k}\right) \frac{1}{\omega_{1}^{2} \omega_{2}^{2}} \\
& +g_{\left(\pi K^{*}\right) ; \Lambda N} f_{\Lambda \Sigma \pi} G_{N \Sigma K^{*}}\left(\boldsymbol{\sigma}_{1} \cdot \mathbf{k} \boldsymbol{\sigma}_{2} \cdot \mathbf{k}_{1}+\boldsymbol{\sigma}_{1} \cdot \mathbf{k}_{1} \boldsymbol{\sigma}_{2} \cdot \mathbf{k}\right) \frac{1}{\omega_{1}^{2} \omega_{2}^{2}} .
\end{aligned}
$$

The configuration space potentials are

$$
V_{\sigma+T}^{(0)}(r)=-2 g_{\left(\pi K^{*}\right) ; \Lambda N} f_{N N \pi} G_{N \Lambda K^{*}}\left(F_{B, \sigma}^{(0)}(r) \sigma_{1} \cdot \sigma_{2}+F_{B, T}^{(0)} S_{12}\right] \mathcal{P}_{f}-2 g_{\left(\pi K^{*}\right) ; \Lambda N} f_{\Lambda \Sigma \pi} G_{N \Sigma K^{*}}\left(F_{B, \sigma}^{(0)}(r) \sigma_{1} \cdot \sigma_{2}+F_{B, T}^{(0)}(r) S_{12}\right] \mathcal{P}_{f}
$$

The nonleading, i.e., $(1 / M)^{1}$, potentials are

$$
\begin{aligned}
\widetilde{V}_{\sigma+T}^{(1)}(\mathbf{q}, \mathbf{k})= & -g_{\left(\pi K^{*}\right), \Lambda N} f_{N N \pi} G_{N \Lambda K^{*}} \frac{1}{2 M_{N}}\left[\left(\sigma_{1} \cdot \mathbf{k} \boldsymbol{\sigma}_{2} \cdot \mathbf{k}_{2}+\boldsymbol{\sigma}_{1} \cdot \mathbf{k}_{2} \boldsymbol{\sigma}_{2} \cdot \mathbf{k}\right)\right] \frac{1}{\omega_{1} \omega_{2}\left(\omega_{1}+\omega_{2}\right)} \\
& -g_{\left(\pi K^{*}\right) ; \Lambda N} f_{\Lambda \Sigma \pi} G_{N \Sigma K^{*}} \frac{1}{M_{\Lambda}+M_{\Sigma}}\left(\boldsymbol{\sigma}_{1} \cdot \mathbf{k} \boldsymbol{\sigma}_{2} \cdot \mathbf{k}_{2}+\boldsymbol{\sigma}_{1} \cdot \mathbf{k}_{2} \boldsymbol{\sigma}_{2} \cdot \mathbf{k}\right) \frac{1}{\omega_{1} \omega_{2}\left(\omega_{1}+\omega_{2}\right)}
\end{aligned}
$$

The configuration space potentials are

$$
\begin{aligned}
V_{\sigma+T}^{(1)}(r)= & +2 g_{\left(\pi K^{*}\right) ; \Lambda N} f_{N N \pi} G_{N \Lambda K^{*}} \frac{m_{\pi}}{2 M_{N}}\left(G_{B, \sigma}^{(1)}(r) \sigma_{1} \cdot \sigma_{2}+G_{B, T}^{(1)}(r) S_{12}\right] \mathcal{P}_{f} \\
& +2 g_{\left(\pi K^{*}\right) ; \Lambda N} f_{\Lambda \Sigma \pi} G_{N \Sigma K^{*}} \frac{m_{\pi}}{M_{\Lambda}+M_{\Sigma}}\left(G_{B, \sigma}^{(1)}(r) \sigma_{1} \cdot \sigma_{2}+G_{B, T}^{(1)}(r) S_{12}\right] \mathcal{P}_{f} .
\end{aligned}
$$

Above, $\mathcal{P}_{f}$ is the flavor-exchange operator, discussed in Refs. $[13,24]$. In addition, we have to multiply these potentials with the isoscalar factors appearing in the Hamiltonian (4.26). For example, for $K-\rho$ and $K-\phi$ pairs, this factor is $+\sqrt{3} / 4$, respectively, $-1 / 4$.

\section{APPENDIX D: EXCHANGE POTENTIALS}

In this section, we follow our multichannel description formalism in the treatment of the exchange potentials [8].

In the case of the antisymmetric spin orbit, the exchange potential requires some attention, because of its special fea- tures. The potentials in configuration space are described in Pauli-spinor space as follows:

$$
\begin{aligned}
V= & V_{C}+V_{\sigma} \boldsymbol{\sigma}_{1} \cdot \boldsymbol{\sigma}_{2}+V_{T} S_{12}+V_{\mathrm{SLS}} \mathbf{L} \cdot \mathbf{S}_{+} \\
& +V_{\mathrm{ALS}} \mathbf{L} \cdot \mathbf{S}_{-}+V_{Q} Q_{12} .
\end{aligned}
$$

Here, the matrix elements of the spin operators are defined as follows:

$$
\left(\chi_{m^{\prime}}^{\dagger}(\Lambda) \chi_{n^{\prime}}^{\dagger}(N)\left|\sigma_{1} \cdot \sigma_{2}\right| \chi_{m}^{\dagger}(\Lambda) \chi_{n}^{\dagger}(N)\right) \equiv\left(\chi_{m^{\prime}}^{\dagger}(\Lambda)\left|\sigma_{1}\right| \chi_{m}^{\dagger}(\Lambda)\right)
$$




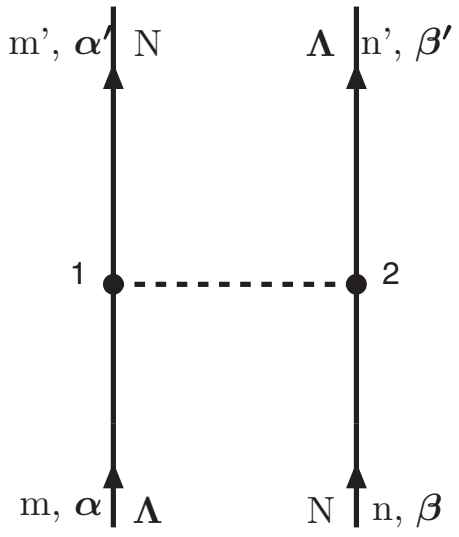

FIG. 8. Particle and spin exchange for $\Lambda N$.

$\left(\chi_{n^{\prime}}^{\dagger}(N)\left|\sigma_{1}\right| \chi_{n}^{\dagger}(N)\right)$, and similarly for the $\mathrm{SU}(2)$ and $\mathrm{SU}(3)$ operator matrix elements. In Fig. 8 , the labels $\left(m, n, m^{\prime}, n^{\prime}\right)$ refer to the spin and the labels $\left(\alpha, \beta, \alpha^{\prime}, \beta^{\prime}\right)$ refer to unitary spin, like SU(2) or SU(3). The momenta on line 1 are $\mathbf{p}$ and $\mathbf{p}^{\prime}$ for respectively the initial and final states. Likewise, the momenta on line 2 are $-\mathbf{p}$ and $-\mathbf{p}^{\prime}$ for respectively the initial and final states.

In Fig. 8, we encounter the matrix elements

$$
\begin{aligned}
\left(\sigma_{1}\right)_{m^{\prime}, m} & =\left(\chi_{m^{\prime}}^{\dagger}(N)\left|\sigma_{1}\right| \chi_{m}^{\dagger}(\Lambda)\right), \\
\left(\sigma_{2}\right)_{n^{\prime}, n} & =\left(\chi_{n^{\prime}}^{\dagger}(\Lambda)\left|\sigma_{2}\right| \chi_{n}^{\dagger}(N)\right) .
\end{aligned}
$$

\section{Spin-exchange potentials}

In order to project the strangeness exchange potentials in Fig. 9 on the forms in Eq. (D1), we have to rewrite these matrix elements in terms of those occurring in Eq. (D2). This

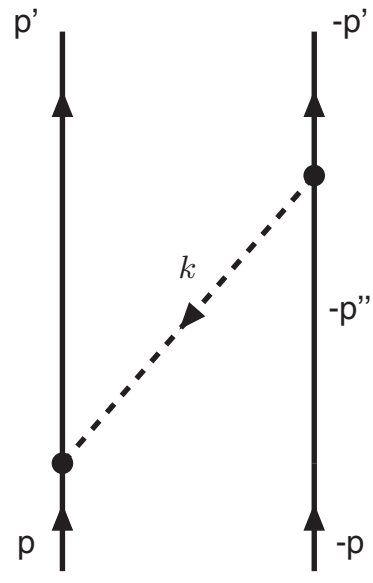

(a)

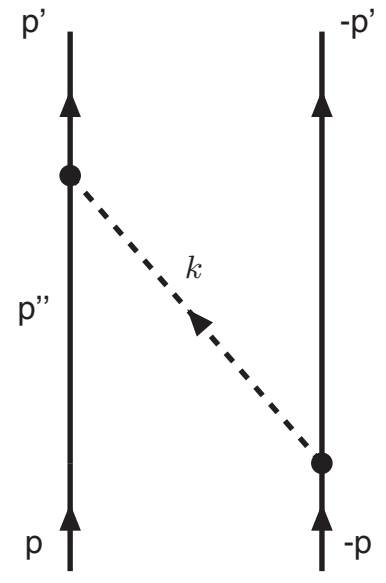

(b)
FIG. 9. $K$ and $K^{*}$ exchange time-ordered graphs (a) and (b). can be done using the spin-exchange operator $P_{\sigma}$ :

$$
P_{\sigma}=\frac{1}{2}\left(1+\sigma_{1} \cdot \sigma_{2}\right) \text {. }
$$

Properties of this operator are

$$
\begin{aligned}
P_{\sigma} \dagger & =P_{\sigma}, \quad P_{\sigma}^{2}=1, \\
P_{\sigma} \chi_{1, m} \chi_{2, n} & =\chi_{1, n} \chi_{2, m}, \\
P_{\sigma} \sigma_{1, k} P_{\sigma} & =\sigma_{2, k}, \\
P_{\sigma} \sigma_{2, k} P_{\sigma} & =\sigma_{1, k} .
\end{aligned}
$$

Similar properties hold for the flavor-exchange operator $P_{f}$, but now for the $S U(2)$ isospin operators $\tau_{k}$, or the $S U(3)$ octet operators $\lambda_{k}$.

In the following, we make only explicit the spin labels, but similar operations apply to the SU(2) or SU(3) labels.

Using this spin-exchange operator, we find that

$$
\begin{aligned}
& {\left[\chi_{1, m^{\prime}}^{\dagger}(N) \chi_{2, n^{\prime}}^{\dagger}(\Lambda)\left|\sigma_{1} \otimes 1_{2}-1_{1} \otimes \sigma_{2}\right| \chi_{1, m}^{\dagger}(\Lambda) \chi_{2, n}^{\dagger}(N)\right]} \\
& \quad=\left[\chi_{2, n^{\prime}}^{\dagger}(N) \chi_{1, m^{\prime}}^{\dagger}(\Lambda)\left|P_{\sigma}^{\dagger}\left(\sigma_{1} \otimes 1_{2}-1_{1} \otimes \sigma_{2}\right) P_{\sigma} P_{\sigma}\right| \chi_{1, m}^{\dagger}(\Lambda) \chi_{2, n}^{\dagger}(N)\right] \\
& \quad=-\left[\chi_{1, m^{\prime}}^{\dagger}(\Lambda) \chi_{1, n^{\prime}}^{\dagger}(N)\left|\left(\sigma_{1} \otimes 1_{2}-1_{1} \otimes \sigma_{2}\right) P_{\sigma}\right| \chi_{1, m}^{\dagger}(\Lambda) \chi_{2, n}^{\dagger}(N)\right] .
\end{aligned}
$$

Above, we added the subscripts 1 and 2 to indicate explicitly the baryon line that is involved.

\section{Spin- and strangeness-exchange potentials}

In addition to the spin exchange, we also have the flavor-exchange operator $P_{f}$ active here. So, in total we have to apply $-P_{\sigma} P_{f}=P_{x}$, i.e., the space-exchange operator. This latter relation follows from the antisymmetry of the two-baryon states, which implies that only states with $P_{f} P_{\sigma} P_{x}=-1$ are physical. All this implies the following:

(1) For the ALS-potential derived in $K$ exchange, one has in Eq. (D1), considering both spin and flavor exchange, the operator

$$
\mathrm{ALS} \Rightarrow \frac{1}{2}\left(\sigma_{1}-\sigma_{2}\right) \cdot \mathbf{L} P_{x}
$$

(2) For the SLS potential derived in $K$ exchange, one has in Eq. (D1), considering both spin and flavor exchange, the operator $P_{f} P_{\sigma}$, but since

$$
\sigma_{1} \cdot \sigma_{2} \sigma_{1, k}=\sigma_{2, k}+i \epsilon_{k l m} \sigma_{1, l} \sigma_{2, m}, \quad \sigma_{1} \cdot \sigma_{2} \sigma_{2, k}=\sigma_{1, k}+i \epsilon_{k l m} \sigma_{2, l} \sigma_{1, m},
$$

one derives easily that

$$
P_{\sigma}\left(\sigma_{1}+\sigma_{2}\right) \cdot \mathbf{L}=\left(\sigma_{1}+\sigma_{2}\right) \cdot \mathbf{L}
$$


and therefore, similar to (D6) we have, with the inclusion of the flavor labels,

$$
\begin{aligned}
& {\left[\chi_{1, m^{\prime} \alpha^{\prime}}^{\dagger}(N) \chi_{2, n^{\prime} \beta^{\prime}}^{\dagger}(\Lambda)\left|\sigma_{1} \otimes 1_{2}+1_{1} \otimes \sigma_{2}\right| \chi_{1, m \alpha}^{\dagger}(\Lambda) \chi_{2, n \beta}^{\dagger}(N)\right]} \\
& \quad=\left[\chi_{2, n^{\prime} \beta^{\prime}}^{\dagger}(N) \chi_{1, m^{\prime} \alpha^{\prime}}^{\dagger}(\Lambda)\left|P_{f}^{\dagger} P_{\sigma}^{\dagger}\left(\sigma_{1} \otimes 1_{2}+1_{1} \otimes \sigma_{2}\right)\right| \chi_{1, m \alpha}^{\dagger}(\Lambda) \chi_{2, n \beta}^{\dagger}(N)\right] \\
& \quad=\left[\chi_{1, m^{\prime} \alpha^{\prime}}^{\dagger}(\Lambda) \chi_{1, n^{\prime} \beta^{\prime}}^{\dagger}(N)\left|\left(\sigma_{1} \otimes 1_{2}+1_{1} \otimes \sigma_{2}\right) P_{f}\right| \chi_{1, m \alpha}^{\dagger}(\Lambda) \chi_{2, n \beta}^{\dagger}(N)\right] .
\end{aligned}
$$

So, for the SLS potential derived in $K$ exchange, one has in Eq. (D1), considering both spin and flavor exchange, the operator

$$
\mathrm{SLS} \Rightarrow \frac{1}{2}\left(\sigma_{1}+\sigma_{2}\right) \cdot \mathbf{L} P_{f}
$$

This treatment for the SLS potential also applies to the central, spin-spin, tensor, and quadratic spin-orbit potentials as well, of course.

We conclude this section by noticing that we have found, using our multichannel setup, the same prescriptions for the treatment of the flavor-exchange potentials as in Ref. [24]. For the treatment of the ALS potential for $S= \pm 1$ exchange, our prescription here is more clear. For example, in the case of the coupled ${ }^{1} P_{1}{ }^{-3} P_{1}$ system, our prescription is unambiguous and given by the $P_{x}$ operator, which is the same for both partial waves coupled in this case.

\section{APPENDIX E: DERIVATION ALS POTENTIALS FOR STRANGE-MESON EXCHANGES}

The contributions to the $P_{8}$-spinor invariant, see Ref. [25],

$$
P_{8}=2\left(\sigma_{1} \cdot \mathbf{q} \sigma_{2} \cdot \mathbf{k}-\sigma_{1} \cdot \mathbf{k} \sigma_{2} \cdot \mathbf{q}\right),
$$

for $\left(K, K^{*}\right)$ exchange were given by Brown, Downs, and Iddings (BDI) [17]. Here, we derive these for $\left(K, K^{*}\right)$, and in particular for the pseudoscalar $K$ within the pseudoscalar (PS) and pseudovector (PV) theory.

\section{1. $K$-exchange ALS potential (PS-PV Theory)}

We derive the $K$-exchange potential using the PV theory and show that we get the BDI answer for the antisymmetric spin-orbit potential (ALS). For graph (a) we get from the vertices the matrix element

$$
\begin{aligned}
\text { (a) } & :-\frac{f_{P}^{2}}{m_{\pi}^{2}}\left[\sigma_{1} \cdot \mathbf{k}+\frac{2 \omega}{M_{\Lambda}+M_{N}} \boldsymbol{\sigma}_{1} \cdot \mathbf{q}\right]\left[-\boldsymbol{\sigma}_{2} \cdot \mathbf{k}+\frac{2 \omega}{M_{\Lambda}+M_{N}} \boldsymbol{\sigma}_{2} \cdot \mathbf{q}\right] \frac{1}{2 \omega} \frac{-1}{\omega-a} \\
& =-\frac{f_{P}^{2}}{m_{\pi}^{2}}\left[\boldsymbol{\sigma}_{1} \cdot \mathbf{k} \boldsymbol{\sigma}_{2} \cdot \mathbf{k}-\frac{2 \omega}{M_{\Lambda}+M_{N}}\left(\boldsymbol{\sigma}_{1} \cdot \mathbf{k} \boldsymbol{\sigma}_{2} \cdot \mathbf{q}-\boldsymbol{\sigma}_{1} \cdot \mathbf{q} \boldsymbol{\sigma}_{2} \cdot \mathbf{k}\right)\right] \frac{1}{2 \omega(\omega-a)}, \\
\text { (b) } & :-\frac{f_{P}^{2}}{m_{\pi}^{2}}\left[\sigma_{1} \cdot \mathbf{k}-\frac{2 \omega}{M_{\Lambda}+M_{N}} \boldsymbol{\sigma}_{1} \cdot \mathbf{q}\right]\left[-\boldsymbol{\sigma}_{2} \cdot \mathbf{k}-\frac{2 \omega}{M_{\Lambda}+M_{N}} \boldsymbol{\sigma}_{2} \cdot \mathbf{q}\right] \frac{1}{2 \omega} \frac{-1}{\omega+a} \\
& =-\frac{f_{P}^{2}}{m_{\pi}^{2}}\left[\sigma_{1} \cdot \mathbf{k} \boldsymbol{\sigma}_{2} \cdot \mathbf{k}+\frac{2 \omega}{M_{\Lambda}+M_{N}}\left(\sigma_{1} \cdot \mathbf{k} \boldsymbol{\sigma}_{2} \cdot \mathbf{q}-\boldsymbol{\sigma}_{1} \cdot \mathbf{q} \boldsymbol{\sigma}_{2} \cdot \mathbf{k}\right)\right] \frac{1}{2 \omega(\omega+a)},
\end{aligned}
$$

where $a=M_{\Lambda}-M_{N}$. Summing these contributions gives ${ }^{3}$

$$
\begin{aligned}
\widetilde{V}_{K}(\mathbf{q}, \mathbf{k}) & =-\frac{f_{P}^{2}}{m_{\pi}^{2}}\left[\frac{1}{2 \omega}\left\{\frac{1}{\omega-a}+\frac{1}{\omega+a}\right\} \sigma_{1} \cdot \mathbf{k} \sigma_{2} \cdot \mathbf{k}+\frac{1}{M_{\Lambda}+M_{N}}\left\{\frac{1}{\omega-a}-\frac{1}{\omega+a}\right\}\left(\sigma_{1} \cdot \mathbf{k} \sigma_{2} \cdot \mathbf{q}-\sigma_{1} \cdot \mathbf{q} \sigma_{2} \cdot \mathbf{k}\right)\right] \mathcal{P}_{f} \\
& =-\frac{f_{P}^{2}}{m_{\pi}^{2}}\left[\sigma_{1} \cdot \mathbf{k} \sigma_{2} \cdot \mathbf{k}-2 \frac{M_{\Lambda}-M_{N}}{M_{\Lambda}+M_{N}}\left(\sigma_{1} \cdot \mathbf{k} \sigma_{2} \cdot \mathbf{q}-\sigma_{1} \cdot \mathbf{q} \sigma_{2} \cdot \mathbf{k}\right)\right] \mathcal{P}_{f} \frac{1}{\omega^{2}-a^{2}}
\end{aligned}
$$

We notice that this result corresponds with the answer in the PS-PS theory, all this in the approximation $\left(M_{\Lambda}+M_{N}\right)^{-1}=$ $\left(1 / M_{\Lambda}+1 / M_{N}\right) / 4$. Now, using the definitions in Refs. [13,25], we have

$$
P_{8}=2\left(\sigma_{1} \cdot \mathbf{q} \sigma_{2} \cdot \mathbf{k}-\sigma_{1} \cdot \mathbf{k} \sigma_{2} \cdot \mathbf{q}\right), \quad P_{6}=(i / 2)\left(\sigma_{1}-\sigma_{2}\right) \cdot \mathbf{n}, \quad \mathbf{n}=\mathbf{p} \times \mathbf{p}^{\prime}=\mathbf{q} \times \mathbf{k},
$$

with the relation [17] $P_{8}=-\left(1+\sigma_{1} \cdot \sigma_{2}\right), P_{6}=2 \mathcal{P}_{x} \mathcal{P}_{f} P_{6}$. This leads to the following expression:

$$
\widetilde{V}_{K}(\mathbf{q}, \mathbf{k})=-\frac{f_{P}^{2}}{m_{\pi}^{2}}\left[\sigma_{1} \cdot \mathbf{k} \sigma_{2} \cdot \mathbf{k}+2 \frac{M_{\Lambda}-M_{N}}{M_{\Lambda}+M_{N}} \cdot(i / 2)\left(\sigma_{1}-\sigma_{2}\right) \cdot \mathbf{n} \mathcal{P}_{x} \mathcal{P}_{f}\right] \mathcal{P}_{f} \frac{1}{\omega^{2}-a^{2}}
$$

\footnotetext{
${ }^{3}$ The $\mathcal{P}$ operators occur in the transition to configuration space. In this Appendix, in contrast to elsewhere in this paper, we include the $\mathcal{P}$ operators in the momentum-space formulas only as a reminder.
} 


\section{2. $K^{*}$-exchange ALS potential}

Upon inspection, we find that the only contribution to the $P_{8}$ invariant is given by

$$
\begin{aligned}
\widetilde{V}_{K^{*}}(\mathbf{q}, \mathbf{k}) \approx & \frac{1}{4} \frac{G_{13} G_{24}}{\omega^{2}-a^{2}} \sigma_{1}\left(\frac{\mathbf{p}}{M_{N}}-\frac{\mathbf{p}^{\prime}}{M_{\Lambda}}\right) \sigma_{2}\left(\frac{\mathbf{p}}{M_{\Lambda}}-\frac{\mathbf{p}^{\prime}}{M_{N}}\right) \mathcal{P}_{f} \\
= & \frac{1}{4} \frac{G_{13} G_{24}}{\omega^{2}-a^{2}}\left[\sigma_{1}\left\{\left(\frac{1}{M_{N}}-\frac{1}{M_{\Lambda}}\right) \mathbf{q}-\frac{1}{2}\left(\frac{1}{M_{N}}+\frac{1}{M_{\Lambda}}\right) \mathbf{k}\right\} \sigma_{2}\left\{\left(\frac{1}{M_{\Lambda}}-\frac{1}{M_{M}}\right) \mathbf{q}-\frac{1}{2}\left(\frac{1}{M_{\Lambda}}+\frac{1}{M_{N}}\right) \mathbf{k}\right\} \mathcal{P}_{f}\right. \\
= & \frac{1}{4} \frac{G_{13} G_{24}}{\omega^{2}-a^{2}}\left[\frac{1}{4}\left(\frac{1}{M_{N}}+\frac{1}{M_{\Lambda}}\right)^{2} \sigma_{1} \cdot \mathbf{k} \sigma_{2} \cdot \mathbf{k}-\left(\frac{1}{M_{N}}-\frac{1}{M_{\Lambda}}\right)^{2} \sigma_{1} \cdot \mathbf{q} \sigma_{2} \cdot \mathbf{q}\right. \\
& \left.-\frac{1}{2}\left(\frac{1}{M_{N}^{2}}-\frac{1}{M_{\Lambda}^{2}}\right)\left(\sigma_{1} \cdot \mathbf{q} \sigma_{2} \cdot \mathbf{k}-\sigma_{1} \cdot \mathbf{k} \sigma_{2} \cdot \mathbf{q}\right)\right] \mathcal{P}_{f}
\end{aligned}
$$

which gives the antisymmetric spin-orbit potential

$$
\widetilde{V}_{K^{*}}(\mathbf{q}, \mathbf{k})=\frac{1}{4} \frac{G_{13} G_{24}}{\omega^{2}-a^{2}}\left(\frac{1}{M_{N}^{2}}-\frac{1}{M_{\Lambda}^{2}}\right)(i / 2)\left(\sigma_{1}-\sigma_{2}\right) \cdot \mathbf{n} \mathcal{P}_{x} .
$$

Finally, we mention the relation with a sometimes-used other form for the antisymmetric spin orbit. Namely, we have $\sigma_{1} \cdot \sigma_{2}\left(\sigma_{1} \times \sigma_{2}\right)=-2 i\left(\sigma_{1}-\sigma_{2}\right)-\sigma_{1} \times \sigma_{2}$, so that

$$
\left(\sigma_{1}-\sigma_{2}\right)=i P_{\sigma}\left(\sigma_{1} \cdot \sigma_{2}\right)
$$

[1] M. M. Nagels, Th. A. Rijken, and Y. Yamamoto, Phys. Rev. C 99, 044002 (2019).

[2] T. A. Rijken, M. M. Nagels, and Y. Yamamoto, Prog. Theor. Phys. Suppl. No. 185, 14 (2010).

[3] M. M. Nagels, T. A. Rijken, and Y. Yamamoto, Extendedsoft-core Baryon-Baryon Model ESC08, I. Nucleon-Nucleon Scattering, arXiv:1408.4825 [nucl-th].

[4] M. M. Nagels, T. A. Rijken, and Y. Yamamoto, Extendedsoft-core Baryon-Baryon Model ESC08, II. Hyperon-Nucleon Interactions, arXiv:1501.06636 [nucl-th].

[5] M. M. Nagels, T. A. Rijken, and Y. Yamamoto, Extendedsoft-core Baryon-Baryon Model ESC08, III. S = -2 Hyperonhyperon/nucleon Interactions, arXiv:1504.02634 [nucl-th].

[6] T. A. Rijken, Phys. Rev. C 73, 044007 (2006).

[7] T. A. Rijken and Y. Yamamoto, Phys. Rev. C 73, 044008 (2006).

[8] T. A. Rijken and Y. Yamamoto, Extended-soft-core baryonbaryon model III. $S=-2$ hyperon-hyperon/nucleon interaction, arXiv:nucl-th/0608074.

[9] L. Micu, Nucl. Phys. B 10, 521 (1969); R. Carlitz and M. Kislinger, Phys. Rev. D 2, 336 (1970).

[10] A. Le Yaouanc, L. Oliver, O. Péne, and J.-C. Raynal, Phys. Rev. D 8, 2223 (1973); 11, 1272 (1975).

[11] N. Isgur and J. Paton, Phys. Rev. D 31, 2910 (1985); R. Kokoski and N. Isgur, ibid. 35, 907 (1987).

[12] M. M. Nagels, T. A. Rijken, and J. J. de Swart, Phys. Rev. D 17, 768 (1978).

[13] P. M. M. Maessen, T. A. Rijken, and J. J. de Swart, Phys. Rev. C 40, 2226 (1989).

[14] T. A. Rijken, V. G. J. Stoks, and Y. Yamamoto, Phys. Rev. C 59, 21 (1999).

[15] E. Hiyama, M. Kamimura, T. Motoba, T. Yamada, and Y. Yamamoto, Phys. Rev. Lett. 85, 270 (2000).

[16] O. Hashimoto and H. Tamura, Prog. Part. Nucl. Phys. 57, 564 (2006).
[17] J. T. Brown, B. W. Downs, and C. K. Iddings, Ann. Phys. (NY) 60, 148 (1970).

[18] For a review, see C. Ewerz, The Odderon in Quantum Chromodynamics, arXiv:hep-ph/0306137.

[19] M. Oka, K. Shimizu, and K. Yazaki, Prog. Theor. Phys. Suppl. 137, 1 (2000).

[20] Y. Fujiwara, Y. Suzuki, and C. Nakamoto, Prog. Part. Nucl. Phys. 58, 439 (2007).

[21] R. Tamagaki and H. Tanaka, Prog. Theor. Phys. 34, 191 (1965); R. Tamagaki, ibid. 39, 91 (1968).

[22] J. Dabrowski, Phys. Rev. C 60, 025205 (1999).

[23] H. Noumi, P. K. Saha, D. Abe, S. Ajimura, K. Aoki, H. C. Bhang, T. Endo, Y. Fujii, T. Fukuda, H. C. Guo et al., Phys. Rev. Lett. 89, 072301 (2002).

[24] M. M. Nagels, T. A. Rijken, and J. J. de Swart, Phys. Rev. D 15, 2547 (1977).

[25] J. J. de Swart, M. M. Nagels, T. A. Rijken, and P. A. Verhoeven, Springer Tracts Mod. Phys. 60, 138 (1971).

[26] M. M. Nagels, T. A. Rijken, and J. J. de Swart, Ann. Phys. (NY) 79, 338 (1973).

[27] R. H. Dalitz and F. von Hippel, Phys. Lett. 10, 153 (1964).

[28] C. Itzykson and J-B. Zuber, Quantum Field Theory (McGrawHill, New York, 1980).

[29] T. A. Rijken, Ann. Phys. (NY) 208, 253 (1991).

[30] T. A. Rijken and V. G. J. Stoks, Phys. Rev. C 54, 2851 (1996); 54, 2869 (1996).

[31] R. A. Bryan and A. Gersten, Phys. Rev. D 6, 341 (1972).

[32] M. Ablikim et al. (BES Collaboration), Phys. Lett. B 645, 19 (2007).

[33] M. Gell-Mann, Phys. Rev. 125, 1067 (1962); S. Okubo, Prog. Theor. Phys. 27, 949 (1962); 28, 24 (1962).

[34] M. Ablikim et al. (BES Collaboration), Phys. Lett. B 698, 183 (2011).

[35] V. G. J. Stoks, R. A. M. Klomp, M. C. M. Rentmeester, and J. J. de Swart, Phys. Rev. C 48, 792 (1993). 
[36] R. A. M. Klomp (private communication).

[37] J. K. Ahn et al., Nucl. Phys. A 761, 41 (2005).

[38] J. A. Kadyk, G. Alexander, J. H. Chan, P. Gaposchkin, and G. H. Trilling, Nucl. Phys. B 27, 13 (1971).

[39] Y. Kondo et al., Nucl. Phys. A 676, 371 (2000).

[40] T. Takahashi et al., Phys. Rev. Lett. 87, 212502 (2001).

[41] E. Hiyama, M. Kamimura, T. Motoba, T. Yamada, and Y. Yamamoto, Phys. Rev. C 66, 024007 (2002).

[42] P. Khaustov, D. E. Alburger, P. D. Barnes, B. Bassalleck, A. R. Berdoz, A. Biglan, T. Burger, D. S. Carman, R. E. Chrien, C. A. Davis et al., Phys. Rev. C 61, 054603 (2000).

[43] V. G. J. Stoks, R. Timmermans, and J. J. de Swart, Phys. Rev. C 47, 512 (1993).

[44] V. G. J. Stoks, R. A. M. Klomp, C. P. F. Terheggen, and J. J. de Swart, Phys. Rev. C 49, 2950 (1994).

[45] F. E. Close and R. G. Roberts, Phys. Lett. B 316, 165 (1993).

[46] V. G. J. Stoks and T. A. Rijken, Nucl. Phys. A 613, 311 (1997).

[47] Open-access website, NN-Online [http:nn-online.org]

[48] G. Alexander, U. Karshon, A. Shapira, G. Yekutieli, R. Engelmann, H. Filthuth, and W. Lughofer, Phys. Rev. 173, 1452 (1968).

[49] B. Sechi-Zorn, B. Kehoe, J. Twitty, and R. A. Burnstein, Phys. Rev. 175, 1735 (1968).

[50] F. Eisele, H. Filthuth, W. Fölisch, V. Hepp, E. Leitner, and G. Zech, Phys. Lett. B 37, 204 (1971).

[51] R. Engelmann, H. Filthuth, V. Hepp, and E. Kluge, Phys. Lett. 21, 587 (1966).

[52] V. Hepp and M. Schleich, Z. Phys. 214, 71 (1968).

[53] D. Stephen, Ph.D. thesis, University of Massachusetts, 1970.

[54] T. Inoue et al., Nucl. Phys. A 881, 28 (2012).

[55] Y. Yamamoto and H. Bandō, Prog. Theor. Phys. Suppl. No. 81, 9 (1985).
[56] Y. Yamamoto, T. Motoba, H. Himeno, K. Ikeda, and S. Nagata, Prog. Theor. Phys. Suppl. No. 117, 361 (1994).

[57] Y. Yamamoto, T. Motoba, and T. A. Rijken, Prog. Theor. Phys. Suppl. No. 185, 72 (2010); E. Hiyama, M. Kamimura, Y. Yamamoto, T. Motoba, and T. A. Rijken, ibid. 185, 106 (2010).

[58] Y. Yamamoto, T. Furumoto, N. Yasutake, and T. A. Rijken, Phys. Rev. C 88, 022801(R) (2013).

[59] Y. Yamamoto, T. Furumoto, N. Yasutake, and T. A. Rijken, Phys. Rev. C 90, 045805 (2014).

[60] Y. Yamamoto, T. Furumoto, N. Yasutake, and T. A. Rijken, Eur. Phys. J. A 52, 19 (2016).

[61] R. R. Scheerbaum, Nucl. Phys. A 257, 77 (1976).

[62] T. Gogami et al., Phys. Rev. C 93, 034314 (1995).

[63] D. J. Millener, C. B. Dover, and A. Gal, Phys. Rev. C 38, 2700 (1988).

[64] A. Gal, Prog. Theor. Phys. Suppl. No. 186, 270 (2010).

[65] T. Harada and Y. Hirabayashi, Nucl. Phys. A 759, 143 (2005).

[66] M. Kohno, Y. Fujiwara, Y. Watanabe, K. Ogata, and M. Kawai, Phys. Rev. C 74, 064613 (2006).

[67] M. M. Nagels, T. A. Rijken, and J. J. de Swart, Phys. Rev. D 20 , 1633 (1979).

[68] G. A. Miller, Phys. Rev. C 39, 1563 (1989).

[69] T. A. Rijken and Y. Yamamoto, Proceedings of the IX International Conference on Hypernuclear and Strange Particle Physics, edited by J. Pochodzalla and T. Walcher (Springer, Berlin, 2006), p. 279.

[70] J. Binstock and R. A. Bryan, Phys. Rev. D 4, 1341 (1971).

[71] J. Schwinger, Phys. Rev. D 3, 1967 (1971).

[72] M. M. Nagels, T. A. Rijken, and J. J. de Swart, Phys. Rev. D 12, 744 (1975). 